\title{
DESIGN PRINCIPLES AND PRELIMINARY TESTING \\ OF A MICROPROPULSION ELECTROSPRAY \\ THRUSTER RESEARCH PLATFORM
}

\author{
A Thesis \\ presented to \\ the Faculty of California Polytechnic State University, \\ San Luis Obispo
}

\author{
In Partial Fulfillment \\ of the Requirements for the Degree \\ Master of Science in Aerospace Engineering
}

by

Will Alan McGehee

July 2019 
(C) 2019

Will Alan McGehee

ALL RIGHTS RESERVED 


\section{COMMITTEE MEMBERSHIP}

TITLE: Design Principles and Preliminary Testing of a Micropropulsion Electrospray Thruster Research Platform

AUTHOR: Will Alan McGehee

DATE SUBMITTED: July 2019

COMMITTEE CHAIR: Amelia Greig, Ph.D.

Assistant Professor of Aerospace Engineering

COMMITTEE MEMBER: Kira Abercromby, Ph.D.

Associate Professor of Aerospace Engineering

COMMITTEE MEMBER: Karl Saunders, Ph.D.

Professor of Physics

COMMITTEE MEMBER: $\quad$ Michael Natisin, Ph.D.

Professional Aerospace Research Engineer, AFRL 


\author{
ABSTRACT \\ Design Principles and Preliminary Testing of a Micropropulsion Electrospray Thruster Research Platform \\ Will Alan McGehee
}

\begin{abstract}
The need for micropropulsion solutions for spacecraft has been steadily increasing as scientific payloads require higher accuracy maneuvers and as the use of small form-factor spacecraft such as CubeSats becomes more common. Of the technologies used for this purpose, electrospray thrusters offer performance that make them an ideal choice. Electrosprays offer high accuracy impulse bits at low power and high efficiency, and have low volume requirements. Design choice reasoning and preliminary testing results are presented for two electrospray thruster designs. The first thruster, named the Demonstration thruster, is operated in atmospheric conditions and serves as a highly visible example of the basic concepts of electrospray technology applied to micropropulsion. It features a single capillary needle emitter and the acetone propellant flow is driven actively by a syringe pump. The second thruster, named the Research thruster, is operated in the vacuum environment and is designed for modularity for its expected use in future research efforts. Propellant flow is also driven actively using a syringe pump. Initial configuration of the Research thruster is a linear array of five capillary needle emitters, though testing is conducted with only one emitter in this thesis. Tests using un-doped glycerol and sodium iodide doped glycerol (20\% by weight) are conducted for the Research thruster. Both thruster designs use stainless steel 18 gauge blunt dispensing needles (0.038 in / $0.965 \mathrm{~mm}$ ID) as their emitters. Applied voltage to the emitter(s) relative to the grounded extractor is swept from $2100 \mathrm{~V}$ to $3700 \mathrm{~V}$ for the Demonstration thruster testing and from $4000 \mathrm{~V}$ to $4500 \mathrm{~V}$ for the Research thruster. Currents incident on a collection plate downstream of the emission plume and on the extractors of the thrusters were measured directly with a pico-ammeter. Measurements made during testing of the Demonstration thruster are inconsistent due to charge loss as propellant travels through the air, though currents as high as $5.1 \times 10^{-9} \mathrm{~A}$ on the collection plate and $2 \times 10^{-7} \mathrm{~A}$ on the extractor are recorded. Currents for Research thruster testing using un-doped glycerol were measured as high as $4.9 \times 10^{-8} \mathrm{~A}$ on the collection plate and $5 \times 10^{-9} \mathrm{~A}$ on the extractor, showing an interception rate as high as $17 \%$. Currents using sodium iodide doped glycerol were measured as high as $7 \times 10^{-7} \mathrm{~A}$ on the collection plate. Discussion is given for the visual qualities of cone-jet emission for all testing.
\end{abstract}

Keywords: Electrospray, Colloid, Thruster, Propulsion, Micropropulsion, Electric Propulsion 


\section{ACKNOWLEDGMENTS}

First and foremost, I would like to thank my advisor, Dr. Amelia Greig, for providing the opportunity to work on this project, and all the support she has given me along the way. Additionally, thanks to all the committee members for their contributions. This project would not have been possible without the support and equipment loans organized by Dr. Michael Natisin at the Air Force Research Labs at Edwards Air Force Base. Thanks to the Aerospace Student Fee Committee for providing a portion of the funding required for this project. Thanks to Nolan Uchizono for the advice given on portions of this thesis. Finally, thanks to my family and friends for their support throughout this work. 


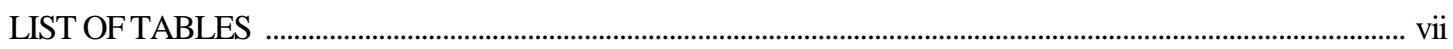

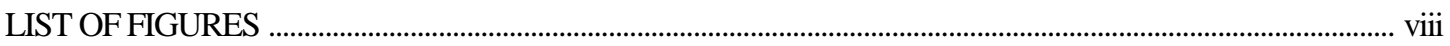

\section{CHAPTER}

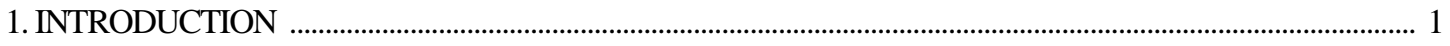

1.1 Importance of Micropropulsion Technology .......................................................................................... 1

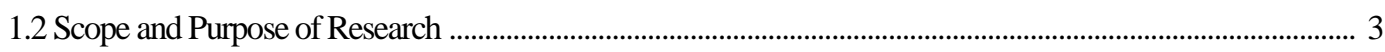

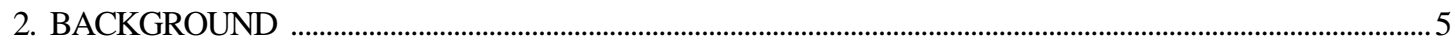

2.1 Physics of Electrosprays ....................................................................................................................

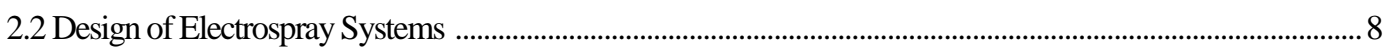

2.3 Electrospray Performance and Diagnostics .................................................................................................... 10

2.4 History and Development of Electrosprays ....................................................................................................... 14

2.5 Recent Electrospray Use .....................................................................................................................

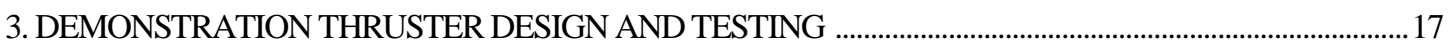

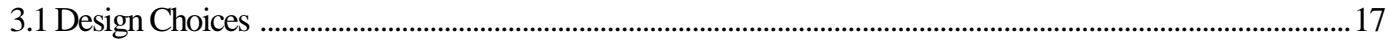

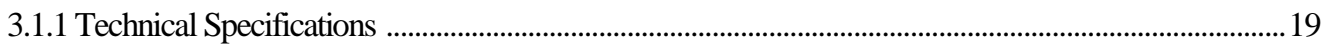

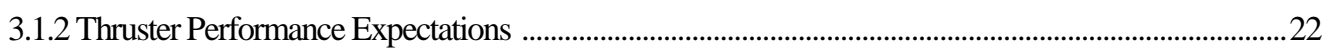

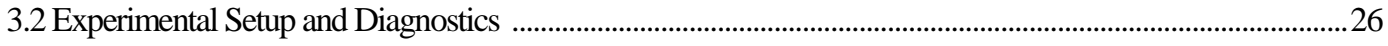

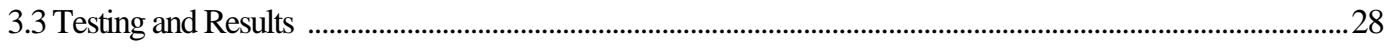

3.3.1 Basic Testing Procedure .....................................................................................................................2

3.3.2 Visual Thruster Operation ...............................................................................................................

3.3.3 Data Results and Discussion .........................................................................................................

4. RESEARCH THRUSTER DESIGN PRINCIPLES ………………………………………………………….......

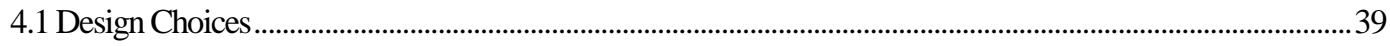

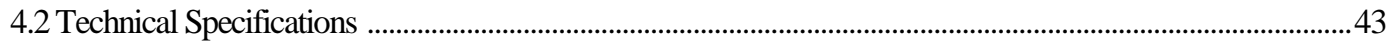

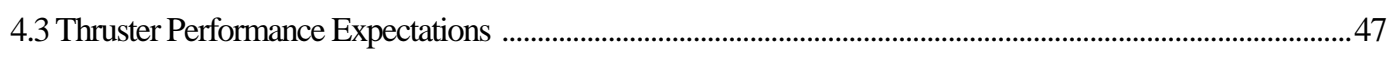

5. RESEARCH THRUSTER TESTING, RESULTS, AND DISCUSSION ……………………………………......51 
5.1 Experimental Setup and Diagnostics ……………………………………………………………………..51

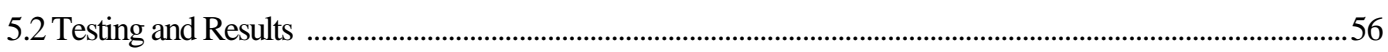

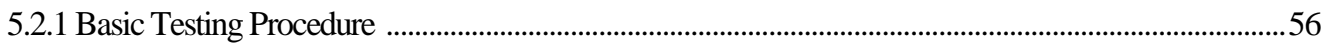

5.2.2 Visual Thruster Operation ............................................................................................................5

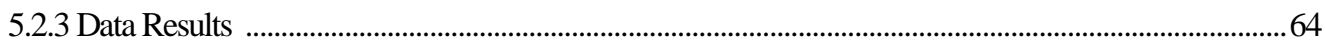

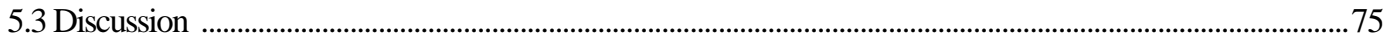

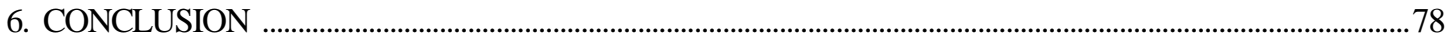

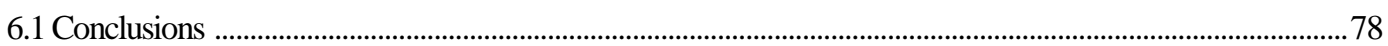

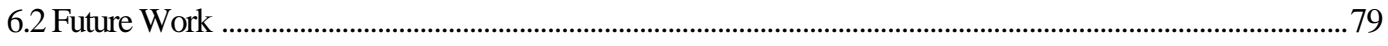

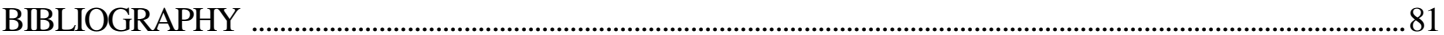

\section{APPENDICES}

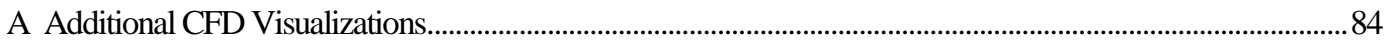

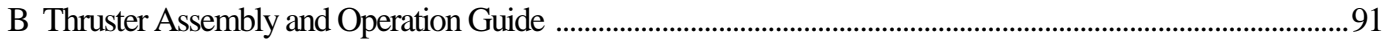

C Sodium Iodide Standard Operating Procedure ....................................................................................................110 


\section{LIST OF TABLES}

Table

Page

5.1 Voltages and Corresponding Current Values from the Second Collector Plate Test of the Research Thruster Using Un-doped Glycerol Propellant.

5.2 Voltages and Corresponding Current Values from the Second Extractor Test of the Research Thruster Using Un-doped Glycerol Propellant.

5.3 Voltages and Corresponding Current Values from the Collector Plate Test of the Research Thruster Using Sodium Iodide Doped Glycerol Propellant. 


\section{LIST OF FIGURES}

Figure

Page

2.1 Simplified Diagram of Basic Electrospray Components and Electrical Biasing.............................................. 6

2.2 Progression of Dripping Emission Mode from an Electrospray. ...........................................................................

2.3 Progression of Unsteady Cone-Jet Emission Mode from an Electrospray. .......................................................... 8

2.4 Progression of Steady Cone-Jet Emission Mode from an Electrospray. .......................................................... 8

2.5 Representations of the Different Physical Types of Emitters Used on Electrosprays: Internally Wetted Capillary (a), Internally Wetted Porous (b), and Externally Wetted Solid (c). .................................... 10

3.1 Assembled 3D Model of the Demonstration Thruster, Showing Major Design Elements. ............................ 18

3.2 Three View Drawing with Major Dimensions and Isometric View of the Assembled Demonstration Thruster.

3.3 Close-Up, Cross Section View of Emitter and Extractor Alignment on the Demonstration Thruster with Major Dimensions.

3.4 Onset Voltage Trend for the Demonstration Thruster Across a Range of Distances Between the Emitter and Extractor.

3.5 Calculated Paschen Breakdown Curve in Air with Relevant Values for the Demonstration Thruster.

3.6 Vector Plot of the Electric Field Strength near the Demonstration Thruster Emitter and Extractor.

3.7 Simplified Electrical Diagram of the Demonstration Thruster. ...........................................................................2

3.8 Block Diagram of Experimental Testing Setup of the Demonstration Thruster Including Electrical Equipment.

3.9 Demonstration Thruster in Its Testing Apparatus with the Syringe Pump.

3.10 Close-Up of the Demonstration Thruster in Its Testing Apparatus Next to the Collection Plate.

3.11 Progression Over 10 Seconds of the Demonstration Thruster Emitting in the Unsteady ConeJet Mode with the Emitter Charged to $2670 \mathrm{~V}$ : Approximately at Relative Times $0 \mathrm{~s}(\mathrm{a}), 3 \mathrm{~s}$ (b), $4 \mathrm{~s}(\mathrm{c}), 5 \mathrm{~s}(\mathrm{~d}), 6 \mathrm{~s}(\mathrm{e})$, and $9 \mathrm{~s}(\mathrm{f})$.

3.12 Progression Over 6 Seconds of the Demonstration Thruster Emitting in the Steady Cone-Jet Mode with the Emitter Charged to $2810 \mathrm{~V}$ : Approximately at Relative Times $0 \mathrm{~s}$ (a), $3 \mathrm{~s}$ (b), and $6 \mathrm{~s}(\mathrm{c})$.

3.13 Steady Cone-Jet Formation of the Demonstration Thruster with the Emitter Charged to Different Voltages: at $2950 \mathrm{~V}$ (a), $3160 \mathrm{~V}$ (b), $3340 \mathrm{~V}$ (c), $3550 \mathrm{~V}$ (d), and $3750 \mathrm{~V}$ (e). .32 
3.14 Comparison of the Demonstration Thruster's Emission Quality Between Tests Where

Current is Collected from the Collector Plate (a) Versus from the Extractor (b).

3.15 Typical Pattern of Propellant Deposited on the Collector Plate After Operating the

Demonstration Thruster.

3.16 Typical Current Data from Collector Plate on the Demonstration Thruster During a Dry

Voltage Sweep.

3.17 Typical Current Data from Extractor on the Demonstration Thruster During a Dry Voltage Sweep.

3.18 Example Current Data from the Collector Plate of the Demonstration Thruster During a Live

Voltage Sweep.

3.19 Example Current Data from the Extractor of the Demonstration Thruster During a Live

Voltage Sweep.

4.1 Assembled 3D Model of the Research Thruster, Showing Major Design Elements.

4.2 Example of Failure During Passive Propellant Feed Testing Due to Expanding Air Bubbles

Causing Excessive Flow out of the Emitters.

4.3 Attempt at Operating All Five Emitters of the Research Thruster at the Same Time Where only Three Emitters Became Active Before the Test Had to be Aborted Due to Arcing.

4.4 Three View Drawing with Major Dimensions and Isometric View of the Assembled Research Thruster.

4.5 Close-Up View of Interchangeable Emitter Block Exploded Assembly on the Research Thruster.

4.6 Close-Up, Cross-Section View of Emitter Array and Extractor Plate on the Research Thruster with Major Dimensions.

4.7 Onset Voltage Trend for the Research Thruster for a Range of Separation Distances Between the Emitters and Extractor.

4.8 Calculated Paschen Breakdown Curve in Air with Relevant Values for the Research Thruster.

4.9 Vector Plot of the Electric Field Strength near the Center Emitter and Extractor for the Research Thruster.

5.1 Simplified Electrical Diagram Connecting the Components of the Research Thruster. . .51

5.2 Vacuum Chamber Used for the Testing of the Research Thruster, Known as "Big Green."

5.3 Block Diagram of Electrical and Propellant Paths and Equipment Used for the Research Thruster Testing.

5.4 Syringe Pump and Propellant Bleed System Connected to Fluid Feedthroughs on the Vacuum Chamber. .54 
5.6 Assembled Complete Testing Apparatus Set up in Vacuum Chamber (a), and Close-Up of the Research Thruster on the Support Structure (b).

5.7 Progression Over 3 Seconds of the Research Thruster Emitting Un-Doped Glycerol in the Unsteady Cone-Jet Mode with the Emitter Charged to $4000 \mathrm{~V}$ : Approximately at Relative Times $0 \mathrm{~s}(\mathrm{a}), 1 \mathrm{~s}(\mathrm{~b}), 1.5 \mathrm{~s}(\mathrm{c}), 2 \mathrm{~s}(\mathrm{~d})$, and $3 \mathrm{~s}(\mathrm{e})$.

5.8 Unsteady Cone-Jet Formation of the Research Thruster Using Un-Doped Glycerol with the Emitters Charged to Different Voltages: at 4000 V (a), 4200 V (b), 4400 V (c), and 4600 V (d).

5.9 Progression Over 4 Seconds of the Research Thruster Emitting Un-Doped Glycerol in the Steady Cone-Jet Mode with the Emitter Charged to 4000 V: Approximately at Relative Times $0 \mathrm{~s}(\mathrm{a}), 2 \mathrm{~s}(\mathrm{~b})$, and $4 \mathrm{~s}(\mathrm{c})$

5.10 Comparison of the Research Thruster's Emission Quality Between Tests Using Un-Doped Glycerol Where Current is Collected from the Collector Plate with the Emitters at 4600 V (a) Versus from the Extractor with the Emitters at $4560 \mathrm{~V}$ (b).

5.11 Two Examples of the Un-Doped Glycerol Propellant Deposited on the Collector Plate by the Research Thruster After Testing.

5.12 Steady Cone-Jet Formation of the Research Thruster Using Doped Glycerol with the Emitters Charged to Different Voltages: at $4000 \mathrm{~V}$ (a), $4150 \mathrm{~V}$ (b), $4300 \mathrm{~V}$ (c), and $4480 \mathrm{~V}$ (d).

5.13 Propellant Deposition Pattern on the Collector Plate from Research Thruster Testing Using Doped Glycerol.

5.14 Typical Raw Data from the Collector Plate on the Research Thruster During a Dry Voltage Sweep.

5.15 Typical Raw Data from the Extractor on the Research Thruster During a Dry Voltage Sweep.

5.16 Raw Data from the Second Collector Plate Test of the Research Thruster During a Live Voltage Sweep.

5.17 Raw Data from the Second Extractor Test of the Research Thruster During a Live Voltage Sweep.

5.18 IV Trace Values Averaged Over Time for the Second Collector Plate Test of the Research Thruster Using Un-Doped Glycerol Propellant.

5.19 IV Trace Values Averaged Across the Peaks in Current for the Second Collector Plate Test of the Research Thruster Using Un-Doped Glycerol Propellant. . .70

5.20 IV Trace Values Averaged Over Time for the Second Extractor Test of the Research Thruster Using Un-Doped Glycerol Propellant.

5.21 IV Trace Values Averaged Across the Peaks in Current for the Second Extractor Test of the Research Thruster Using Un-Doped Glycerol Propellant. 
5.22 Raw Data from the Collector Plate of the Research Thruster During a Live Voltage Sweep Using Sodium Iodide Doped Glycerol Propellant.

5.23 IV Trace Values Averaged Over Time for the Collector Plate Test of the Research Thruster Using Sodium Iodide Doped Glycerol Propellant.

5.24 Propellant Deposition on the Outside Face of the Extractor on the Research Thruster After the Second Collector Plate Test Using Un-Doped Glycerol (a), the Second Extractor Test Using Un-Doped Glycerol (b), and the Collector Plate Test Using Sodium Iodide Doped Glycerol (c).

5.25 Example of Electrical Arcing During a Failure of the Research Thruster Where Propellant Had Bridged the Gap Between the Emitters and the Extractor.

5.26 Comparison Between Before and After Testing of the Needle End on the Most Used Research Thruster Emitter Needle. 


\section{Chapter 1}

\section{INTRODUCTION}

In the recent years of the aerospace industry, there has been a significant shift from large bus satellites to smaller form factor satellites, such as CubeSats. A primary motivation for this change was to reduce the overall cost of developing and launching a satellite into orbit. Rather than develop a large bus which carries a plethora of payloads and many redundancies to reduce the risk of single point failure, a developer can spend less time developing a smaller satellite with potentially reduced capability [1]. A design like this will maintain less overall risk compared to the large design simply due to the relatively large reduction in expenditure of money and manpower. Another advantage is how it opens the opportunity for smaller organizations with likely less manpower and capital to participate in the greater scientific community and make significant contributions by using lower cost, sometimes modular spacecraft designs.

\subsection{Importance of Micropropulsion Technology}

As developers shift focus towards designing smaller satellites, there are some engineering problems that come about related to miniaturizing the internal components while maintaining survivability in space. In addition, the higher usage of commercial-off-the-shelf (COTS) components for spacecraft provides better support during the design phase, so developing standardized components benefits the entire industry over time [2]. In the sense of cost and availability there is the endeavor to standardize as many components of a spacecraft as possible. There has been significant progress made since the advent of the CubeSat standard [3], including electronics, structure, and deployment systems, allowing organizations of many backgrounds to participate in space-based research, such as academic colleges and small startup companies. More involvement in the field of space exploration and earth observation will ultimately increase the availability and support for COTS components, reducing design obstacles typically associated with spacecraft development as the components no longer need to be custom made every time.

As the community and industry surrounding the small satellite grows, so do the technological ambitions of the missions. Currently, these small satellite systems do not typically carry any propulsion systems due to power demand and availability, volume requirements, cost or complexity, or simply because 
the mission does not necessitate propulsion. Recently, ambitions and mission goals have grown to make it so that on-board propulsion is highly sought after to extend the spacecraft's lifetime beyond the natural de-orbit time by compensating for drag and other perturbations, allowing the payloads to continue operating in space longer. Propulsion also would enable different mission types altogether which were previously out of reach when using smaller satellites, such as lunar missions, those involving planetary transfer, or de-orbit operations for space junk or defunct spacecraft that would normally not return to earth.

In pursuit of satisfying the requirements of these missions, many different technologies exist that are being adapted to be used on the small form-factor spacecraft. One example is electrospray technology, which are highly adaptable and often modular, allowing them to be a viable option for a wide range of missions. Electrospray thrusters operate on electrostatic acceleration, where charged particles are accelerated by a static electric field [4]. The electric field is created by biasing electrically isolated components to high potentials. Propellant is delivered into the electric field by individual discreet emitters, where flow is driven either actively or passively. There are many beneficial qualities exhibited by electrosprays. These systems typically have very low power draw, making them ideal for systems with limited power generation. Like most electric propulsion methods, electrosprays have low thrust and higher efficiencies compared to chemical methods, such as cold-gas or combustion [4]. The propellants used are typically liquids, or sometimes even solids, which have a great advantage in storage volume over electric propulsion using inert gases [5]. Of course, as with any method, there are some disadvantages of electrosprays. These systems need to operate at relatively high voltages, on the order of several thousand volts. Physical alignment of the thruster components is extremely important for efficient operation. Additionally, electrospray components will suffer from electro-chemical degradation over time from operation [6], though degradation is common for many electric propulsion technologies [7].

While the technology of electrosprays has been around for some time and utilized in a variety of applications such as mass spectrometry [8], it has seen a resurgence in interest recently when applied to spacecraft propulsion and is seen as a viable option with future potential [5]. As of today, electrospray thrusters have a limited flight heritage. An excellent demonstration of their use in the role of attitude adjustment is the LISA Pathfinder mission launched in 2016 [9]. Technology demonstrations have been launched on CubeSat buses recently, such as the IFM Nano Thruster launched in 2018 [10]. 


\subsection{Scope and Purpose of Research}

The increasing demand for micropropulsion solutions for a variety of different mission uses is driving research into working solutions. Particularly with electrosprays there is an effort to expand research capability among capable institutions, in order to accelerate progress in the field. Recently, electrospray technology and research has expanded quickly in industry and research institutions, with some focusing primarily on producing performance models designed for use on spacecraft as opposed to being purely for research. There is still much left to understand about electrosprays designed for propulsion, such as the fusion of theoretical models and physical testing data to better predict thruster performance during the design phases.

This thesis will encompass the design and initial testing of two electrospray thruster systems. The first is a simple single emitter setup meant to serve as a proof of concept of the technology and operate in atmosphere for quick and easy setup and observation. This single emitter atmospheric thruster is referred to as the "Demonstration thruster" or simply "Demo thruster" throughout this writing. The second electrospray is a more robust design featuring an interchangeable structure capable of accommodating a reasonable amount of emitter array designs. The initial design features a linear array of five emitters, though only one was used for the majority of testing. This thruster is designed to be utilized as a platform for research at $\mathrm{Cal}$

Poly, where future students can make quick adjustments to facilitate a range of research topics, and is referred to as the "Research thruster" throughout this writing.

The content laid out in this thesis will cover the design principles that were the chosen to define the Demonstration and Research thrusters, how the experiments were designed and set up, and what the results show about the operation of the system and its relevance to the greater scientific community. The Demonstration thruster is detailed in chapter 3, and the Research thruster is detailed in chapters 4 and 5. A large part of the legacy of this project will be the knowledge gained of how to realistically fabricate and operate an electrospray thruster using common materials and machining methods. This is something that Cal Poly has never approached as a research avenue, so it follows that there is considerable hands-on understanding to be gained during the first efforts to begin researching. As such, suitable effort and discussion is made to highlight the potential for improvement and future research topics. 
This thesis is aimed primarily at the initial stages of this research's lifetime. The scope is limited to the basic principles of an electrospray design and their initial testing to verify operation. As such, the testing is limited to simply and reasonably proving that the thrusters are operational through measurement of currents created by successful electrospray emission. This confirmation proves that the thrusters will serve their purpose in research. The end result of this thesis is by no means the definitive work surrounding the produced thrusters, nor does it accurately determine the performance that the thrusters are capable of in ideal circumstances. Additional work would be necessary in developing the thruster system to greater maturity. A major goal of this thesis is to start the research program of electrosprays at California Polytechnic State University (Cal Poly), both as novel research efforts and in application, by developing a capable thruster model and the diagnostics necessary to test an electrospray's performance. Cal Poly has an experienced CubeSat program with many deployments of its satellites designed in-house. The program is seeking to develop satellites featuring micropropulsion systems in the future. The lessons of the work described here will serve to provide a starting point for any future endeavors with electrosprays, beyond the physical thrusters produced from this thesis. 


\section{Chapter 2}

\section{BACKGROUND}

The electrospray technology as applied to propulsion has been around since the 1960's, around the same time frame as gridded ion thrusters and Hall effect thrusters [4]. However, their use on spacecraft did not come to be adopted for the missions of the time, because in order to achieve thrusts high enough, the designs required voltages high enough to cause problems with insulation and packaging. Recent developments in fabrication technology, propellants, and the shift towards smaller spacecraft have increased interest in electrospray systems. While the fundamental concept of the electrospray has not changed, the particular designs have. With the advent of micro-machining and the use of higher performance propellants, electrospray designs are attempting to achieve higher thrust densities and performance, in overall smaller modules [5].

\subsection{Physics of Electrosprays}

The electrospray system operates on electrostatics applied to conductive liquid propellants for emission to be achieved. Essentially, the propellant must react to an electric field as this is how particles are extracted and accelerated. For operation in space, it is also necessary that the liquid also have a low vapor pressure so it does not boil away at the extremely low pressures it will be exposed to, but propellants with high vapor pressures will work in atmosphere [4]. A simplified schematic portraying the basic components of a complete electrospray thruster is shown in Figure 2.1. The liquid propellant is subjected to a strong electric field, which is created by charging either the extraction grid (also called the extractor) or the emitters and grounding or oppositely biasing the other. Because the propellant is conductive, the electric field causes it to be attracted towards the extractor. The force imparted by the electric field is balanced in the opposite direction by the surface tension of the liquid itself. When the force from the electric field overpowers the surface tension at the very tip of the propellant, emission is achieved as particles separate from the main body [5]. Once separated, the propellant is accelerated by the electric field and expelled to generate thrust. 


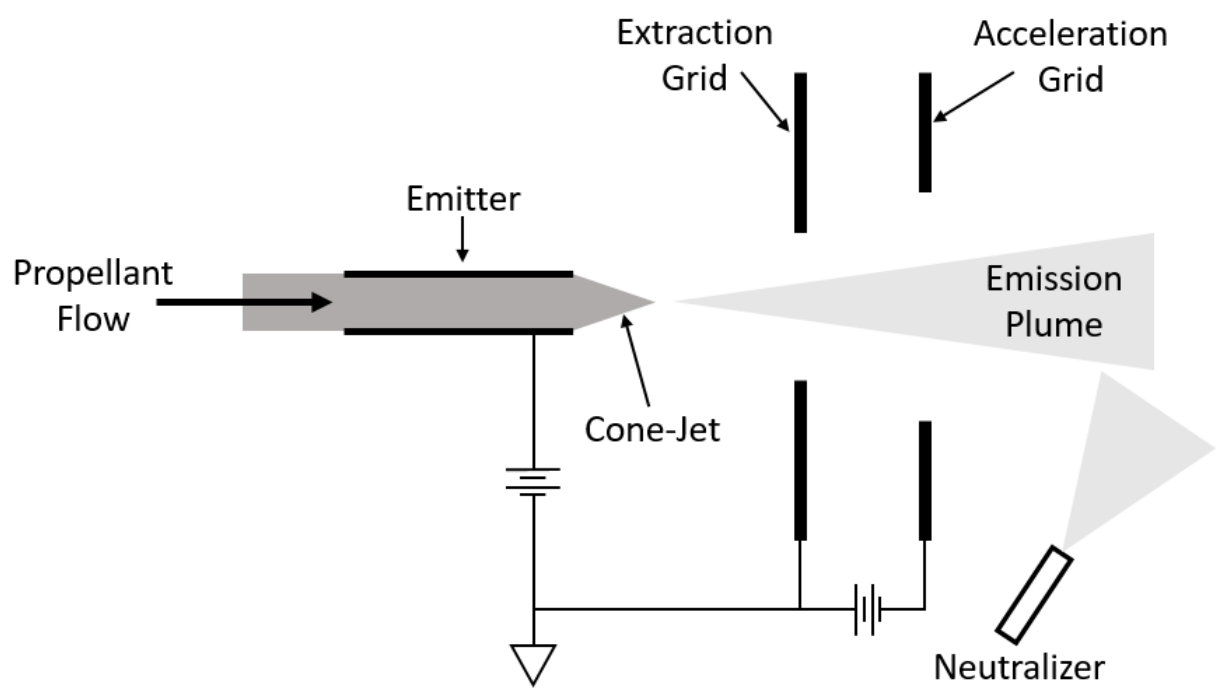

Figure 2.1: Simplified Diagram of Basic Electrospray Components and Electrical Biasing.

The addition of an acceleration grid serves to improve performance by increasing the exit velocity of the propellant particles, and thus the thrust and specific impulse, though this grid is not essential for emission. A neutralizer would need to be included for in-space operation to create a quasi-neutral emission plume so that the charged particles are not attracted back to any portion of the spacecraft, which negates the thrust they would have generated as well as potentially causing charging or arcing issues on the spacecraft itself. For laboratory applications and testing, the neutralizer is generally not used because the charge is necessary to impart a current on collection surfaces. Once the charged emissions hit the collection surface and the electrical charge goes to ground, the propellant will not be attracted to any other conductive parts on the thruster. In this way, the collection surface neutralizes the propellant emissions, though neutralization is not necessary for successful emission.

The region where the propellant emits from the main body is a theoretically ideal phenomenon known as a Taylor cone $[5,11]$. Technically, the formation of a proper Taylor cone necessitates that there is no fluid flow at all, so in reality where there is indeed flow, this formation is referred to as a cone-jet [11]. How a cone-jet forms determines the quality of emission from the emitters, and thus greatly affects the overall performance of the thruster. A cone-jet can be stable, where the propellant is constantly emitting and the shape of the cone does not change over time at the same extraction voltage, or unstable, where the cone-jet will intermittently form and emission is inconsistent over time at the same extraction voltage. The formation 
of the cone-jet depends on several factors of the thruster's operation, including the physical and electrical properties of the propellant, the type and dimensions of the emitter the propellant is flowing through, and the applied electric field. The cone-jet formation is primarily determined by the potential difference between the emitters and the extractor [11]. In addition to the formation of the cone-jet itself, the emissions extracted will either be droplets consisting of many molecules of propellant, or ions consisting of single molecules of propellant. Electrosprays that emit primarily droplets will have lower efficiencies and higher thrusts as the relatively large mass droplets are accelerated to lesser velocities [12]. Those that emit primarily ions will have higher efficiencies and lower thrusts as the low mass ions are accelerated to higher velocities. For both emission types, differences in the mass of the droplets and ions will affect thrust and efficiencies.

A cone-jet is the ideal emission mode for electrospray thrusters, though propellant can be extracted without one forming. The dripping emission mode is a phenomenon where relatively large volumes of propellant are removed from the main body of propellant in the extractor and accelerated by the electric field [13]. An example of this process over time is shown in Figure 2.2. This mode can also include cone-jet formations on the large droplet that is being separated. The unsteady cone-jet mode of operation is when the propellant at the tip of the emitter oscillates between a cone-jet and a blunted shape [13]. In this mode, emission only occurs when the cone-jet is formed, and so the current output will oscillate between a higher current than would be seen with a stable cone-jet and zero [11]. An example of this oscillation over time can be seen in Figure 2.3 [13]. Stable cone-jet emission is characterized by consistent emission through a Taylor cone over time. Operating in this mode would produce a steady current rather than short frequent bursts. An example of this operational mode can be seen in Figure 2.4.

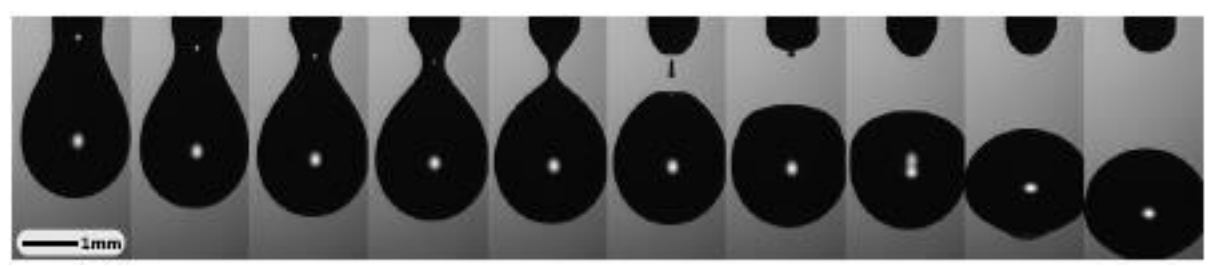

Figure 2.2: Progression of Dripping Emission Mode from an Electrospray. 


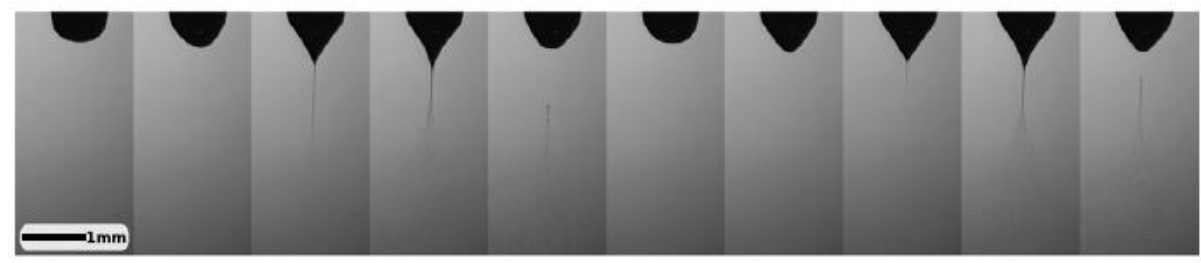

Figure 2.3: Progression of Unsteady Cone-Jet Emission Mode from an Electrospray.

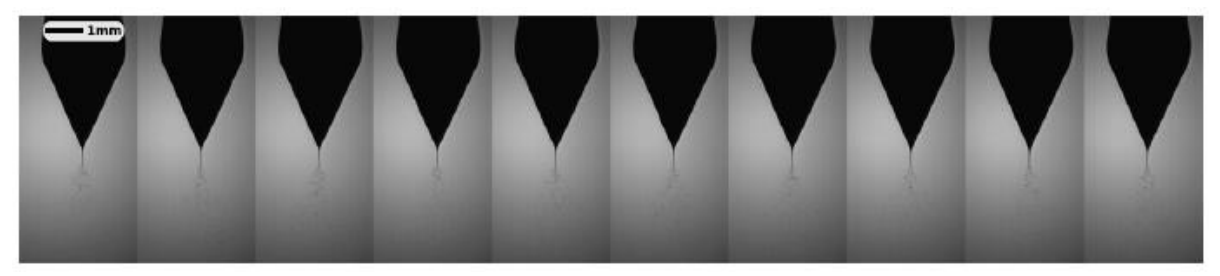

Figure 2.4: Progression of Steady Cone-Jet Emission Mode from an Electrospray.

Common propellants used are liquid metals, ionic liquids, and conductive solvents [4, 5]. The choice of which propellant to use depends on a number of factors such as the physical design of the thruster, physical properties of the propellants, and performance demands. Depending on the propellant used and the specific types of physical phenomenon observed during operation, the thruster will be classified as a specific type of electrospray [5]. Colloids make use of potentially chemically doped solvents such as glycerol and primarily operates in the droplet regime. Field emission electric propulsion (FEEP) thrusters utilize liquid metals and operate primarily in the ionic emission regime. Ionic liquid ion sources (ILIS) thrusters make use of ionic liquids (also known as liquid salts) and ideally operates in the ionic regime, but can produce mixtures of ions and droplets. The focus of this section will be placed on colloids as they are most pertinent to the designs featured in this thesis.

\subsection{Design of Electrospray Systems}

The specific design elements that an electrospray thruster will incorporate will depend significantly on the application, be it for performance or for research. Designs for performance can be driven by seeking the largest thrust density, necessitating densely packed arrays of emitters, or by seeking high accuracy small impulse bits for high precision maneuvers, needing only few emitters total [4]. Arrays can consist of a handful of emitters to a two-dimensional array consisting of hundreds of discrete emitters, lending to the modularity 
and scalability of the technology. Research designs are more focused on the particular phenomenon the researchers are attempting to study, and so can vary significantly. Single emitter thrusters are primarily seen in research settings because of their simplicity $[14,15]$. The major dimensions are typically driven by the number of emitters making up the emitter array, but other factors such as making the workings visible do contribute.

There are several types of emitter that serve to transport the propellant to be emitted. Visual representations for each of these types of emitters can be found in figure 2.5. The role of the emitter is to deliver the propellant into the electric field. If the flow is driven passively, then the emitter must also control the flow rate of propellant to the emission sites. The flow rate of propellant is crucial to the proper and efficient operation of each emitter [16]. Commonly used for colloids is the internally wetted capillary emitter, where the propellant simply flows through a hollow needle, such as the studies of F. M. Pranajaya and M. A. Cappelli [14], and the colloid flown aboard LISA Pathfinder in 2016 [9]. Typically thrusters utilizing an internally wetted capillary needle emitter will have propellant flow actively driven rather than relying on passive capillary action, but passive flow is possible. Internally wetted porous emitters are more commonly used today for performance models due to the capillary action driving flow passively, such as the commercial designs of Busek Co., Inc. [17] and Accion Systems Inc. [18], or the recently flown IFM thruster [10]. The pores at the tip of the emitter are sites where cone-jets can form and emit, and propellant flows through the interconnected cells throughout the material. Lastly, externally wetted emitters function by having propellant wet itself over the external surface of a solid needle or protrusion and are more commonly seen with FEEP thrusters and metallic propellants due to their better wetting characteristics, such as the electrospray thruster being developed at JPL that uses liquid indium [19]. 


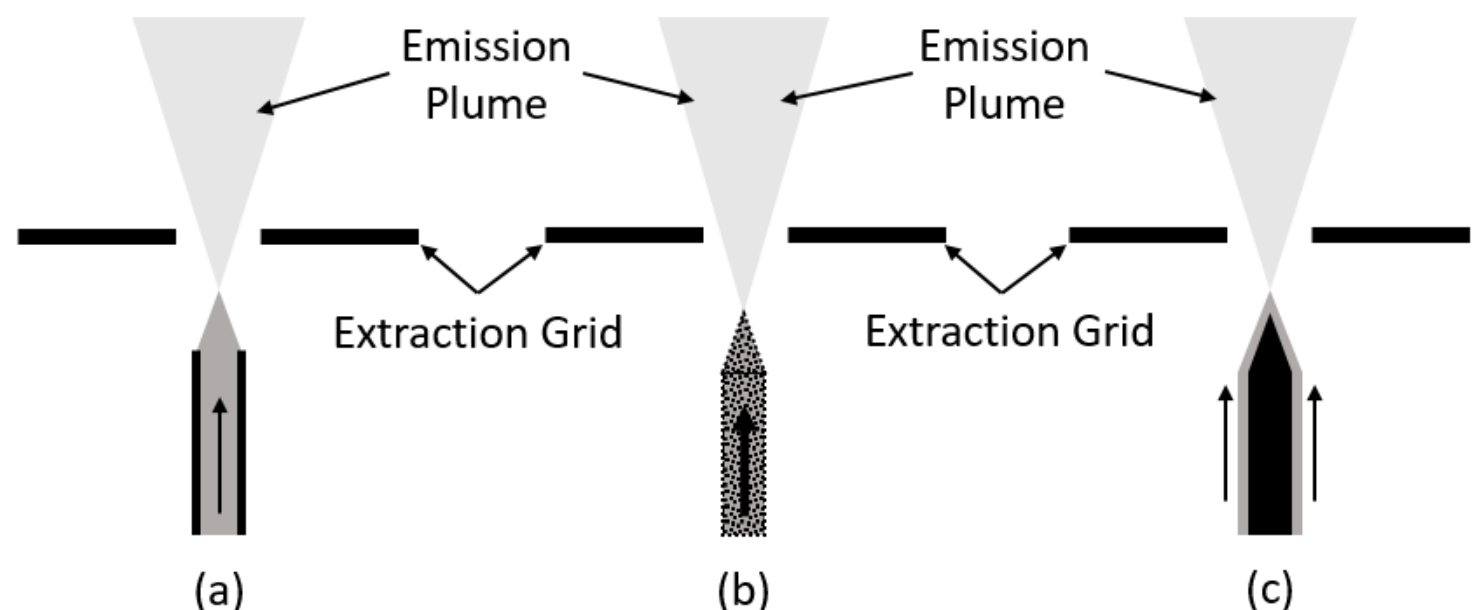

Figure 2.5: Representations of the Different Physical Types of Emitters Used on Electrosprays: Internally Wetted Capillary (a), Internally Wetted Porous (b), and Externally Wetted Solid (c).

\subsection{Electrospray Performance and Diagnostics}

The practice of characterizing the performance of an electrospray system is heavily focused on collecting or separating the charged particle emissions. Methods of gathering data can be as simple as a conductive collection plate to intercept the emission plume [14]. The charge particles incident on the collector plate will create a measureable current that will relate to key performance metrics of the thruster. Particularly, if the collector plate captures the entirety of the emission plume, the current measured will be the beam current. Knowing the mass flow rate of propellant and the beam current, performance metrics such as thrust specific impulse, and efficiency. The charge over mass ratio for the particles of propellant emitted from the thruster can be estimated using equation 2.1, where $q$ is the total charge on an individual particle, $m$ is the mass of an individual particle, $I_{b}$ is the beam current, and $\dot{m}$ is the mass flow rate of propellant [14].

$$
\frac{q}{m}=\frac{I_{b}}{\dot{m}}
$$

Using the charge over mass ratio, the exit velocity $v_{e}$ of the propellant particles can be found using equation 2.2, where $\phi$ is the potential difference that is experienced by the travelling particle [14].

$$
v_{e}=\sqrt{2 \frac{q}{m} \phi}
$$


The thrust $T$ generated by the electrospray can be calculated using equation 2.3 [14]. To increase the thrust of an electrospray system, the propellant mass flow rate can be increased to increase the amount of particles that are accelerated through the potential difference, or the potential difference can be increased to accelerate the particles to higher velocity.

$$
T=\dot{m} v_{e}
$$

The specific impulse $I_{s p}$ can be calculated using equation 2.4 , where $g$ is the gravity acceleration constant at sea level on Earth [14]. The only direct way to increase the specific impulse is by increasing the exit velocity of the propellant particles, which in turn can only be increased by increasing the potential difference created by the grids.

$$
I_{s p}=\frac{v_{e}}{g}
$$

Recall that electrosprays can emit in both the droplet and ionic modes. When an electrospray thruster is extracting in the droplet mode, more thrust will be generated relative to the ionic mode as the mass flow rate is much larger despite the exit velocity being decreased, referring to equation 2.3. Because the velocity is decreased, the droplet mode emission will also have a lower specific impulse, referring to equation 2.4. During ionic mode emission, the ions have very small mass and so are accelerated to faster velocities, but the mass flow rate decreases enough to produce an overall lower thrust compared to droplet emissions, referring to equation 2.3. The higher exit velocities of the ions results in a higher specific impulse as well, referring to equation 2.4.

Note that equations 2.1 to 2.4 are the ideal representations of the performance of an electrospray thruster. In reality, the thruster's performance will deviate from the ideal cases as the particles in the emission beam are not consistent. The mass and charge of each particle can vary drastically between droplets and individual ions, creating a range of charge over mass ratios that need to be accounted for through integration of a distribution. This distribution can be found experimentally through certain diagnostics, but is difficult to predict theoretically. There are also inconsistencies with electrical equipment that can't be avoided, such as fluctuating voltages being applied to the thruster components, which will affect the potential experienced by the charged emission particles, and subsequently affect the velocity the particles are accelerated. 
Measuring the current incident on the extraction grid or any other grid downstream will reveal the interception occurring during thruster operation. Interception is the issue with propellant particles that are extracted from the emitter but don't contribute to thrust because the impact the downstream grids of the thruster: the extraction grid or the acceleration grid. The current lost in this way leads to the primary efficiency loss as the intercepted emissions do not contribute to thrust. The system efficiency $\eta$ is calculated in equation 2.5, where $P_{\text {emission }}$ is the power used to emit the propellant that contributes to the beam current, defined in equation 2.6, and $P_{\text {loss }}$ is the power used to emit the propellant that contributes to the intercepted current, $I_{\text {loss }}$, defined in equation 2.7 [14]. Since the grids are biased to a stable voltage, any incident current needs to be compensated by the power supply, resulting in additional power usage that otherwise would not be necessary.

$$
\begin{gathered}
\eta=\frac{P_{\text {emission }}}{P_{\text {emission }}+P_{\text {loss }}} \\
P_{\text {emission }}=\phi I_{b} \\
P_{\text {loss }}=\phi I_{\text {loss }}
\end{gathered}
$$

Calculating the onset voltage is useful to determining a guide for how much voltage is necessary to apply to the system in order to achieve emission. Onset voltage refers to the minimum potential difference applied to the propellant necessary to achieve emission; the onset of current escaping the emitter. The calculation requires only a few key characteristics of the system. The derivation of these relationships makes assumptions of the geometry of the physical phenomenon, and the equations can be simplified even further. Equation 2.8 defines the simplified relationship that served as the baseline for the thrusters designed in this thesis $[12,16]$, where $V_{o c}$ is the onset voltage, $\gamma$ is the surface tension of the propellant, $r_{t}$ is the radius of the emitter tip, $\varepsilon_{0}$ is the permittivity of free space constant, and $d$ is the distance between the emitter and the extractor.

$$
V_{o c}=\sqrt{\frac{\gamma r_{t}}{\varepsilon_{0}}} \ln \left(\frac{4 d}{2 r_{t}}\right)
$$


Another common derivation of this relationship can be found using slightly different parameters, such as a different denominator in the natural log, as seen in equation $2.9[5,20]$.

$$
V_{o c}=\sqrt{\frac{\gamma r_{t}}{\varepsilon_{0}}} \ln \left(\frac{4 d}{r_{t}}\right)
$$

These equations are simplifications using certain geometric assumptions, and so they don't describe every system perfectly. Because these equations aren't used for any rigorous analysis for this thesis and just serve as guidelines, the resulting calculations were treated as bounds for expected emission. Equation 2.8 proved to be more accurate in predicting emission in the droplet regime for this thesis $[12,16]$. Theoretically, this calculation predicts the minimum voltage necessary to achieve emission with the given distance between the emitter and extractor. However, emission can and will occur at lower voltages as well. This will occur as the dripping emission mode discussed in section 2.1 .

To ensure safe operation, an analysis of the breakdown voltage threshold must be compared to the expected operational voltages. Equation 2.10 states the theoretical relationship, where $V_{B}$ is the breakdown voltage, $A$ is the saturation ionization, $B$ is related to excitation and ionization energies, $p$ is the pressure, $d$ is the distance between electrodes, and $\gamma_{s e}$ is the secondary electron emission coefficient [21].

$$
V_{B}=\frac{B p d}{\ln (A p d)-\ln \left[\ln \left(1+\frac{1}{\gamma_{s e}}\right)\right]}
$$

A useful diagnostic for defining how an electrospray thruster is operating is the retarding potential analyzer (RPA) [22]. This technology involves using a series of three or four biased grids to selectively sift through the charged particles to determine ion energy distribution. The first grid is either grounded or set to the plasma's floating potential to prevent the biasing of the other grids from influencing the plasma as it approaches the RPA. The second grid is biased to high negative voltage to prevent electrons from passing while allowing passage of the ions. The third grid is swept along a range of positive voltages where only ions with energy above a corresponding threshold will pass through. A fourth optional grid is biased to negative voltage to prevent any stray electrons from reaching the inside of the RPA. Ions that reach the inside hit a conductive plate and the impact creates a measureable current. 
The time of flight (ToF) diagnostic is another method of characterizing thruster performance by determining the emission particle's charge to mass ratio more accurately [23]. This method involves the use of gates which allow the emission plume to pass for only a short controlled time. After the plume is allowed through, the emissions will impact a collector plate a precisely known distance away. The ratio of the particle's charge to its mass will determine how that particle is accelerated by the applied potential difference on the thruster's grids and emitters. This is revealed by the ToF technique because particles of different mass will impact the collector plate at different times and in different quantities, which relates to currents measured on the collector. Knowing this value directly allows the thruster's performance metrics, such as thrust, to be more accurately calculated using integral forms of equations 2.1-2.4.

\subsection{History and Development of Electrosprays}

The earliest known example of electrosprays is in 1750 when physicist Jean-Antoine (Abbé) Nollet successfully aerosolized water by subjecting it to an electric field [8]. Interestingly, he also noted that electrified blood would spray out from an open wound when that body was connected to a high voltage generator. Lord Kelvin conducted experiments with the concepts of electrosprays later in the $19^{\text {th }}$ century. The beginnings of electrosprays as used today in the fields of mass spectrometry and propulsion are with the work done by John Zeleny and G. I. Taylor in the early $20^{\text {th }}$ century. Zeleny photographed operational ethanol electrosprays where the liquid formed into a conical shape and subsequently emitted a spray of particles. This cone was late coined as the Taylor cone after Taylor's theoretical work on the subject matter. From then on, some professions have made use of the phenomenon, such as painting, before the concept was applied for scientific purposes.

Development of the electrospray technology as applied to spacecraft propulsion dates back into the

1960's $[4,5]$. The goal was to apply the thrusters as attitude control or drag compensation for larger spacecraft. A large part of the research done at this time was with colloid thrusters utilizing glycerol doped with various chemicals as a propellant. With particular dopants, either polarity of emissions can be achieved while maintaining the beneficial properties of the main solvent. Studies have been conducted on capitalizing on the bi-polar capabilities of these propellants by designing thrusters with the ability to produce both 
polarities of emission, such as the work done by J. Perel, et al. in 1967 [24]. Though the thrusters worked, there were numerous issues that came from the specific applications, such as their inability to produce enough thrust at reasonable power levels. Acceleration voltages as high as $20 \mathrm{kV}$ could be demanded, resulting in a system that was increasingly difficult to package while maintaining electrical isolation and thrust density. For these reasons, the pursuit was all but abandoned in favor for other alternatives at the time.

Today, electrosprays are being actively developed and research with the rising interest and successes of smaller satellites, along with development of fabrication techniques and additional suitable propellants [4, 5]. Higher emitter densities and thus thrust densities can be achieved in smaller, lower power systems. In addition to improving the colloid and FEEP thrusters, recent technology advances have enabled the ILIS systems to be produced more reliably, offering more opportunities for electrospray propulsion. As efforts continue to increase, the industry has seen many performance models developed alongside research efforts.

\subsection{Recent Electrospray Use}

There are currently many organizations working to better understand electrosprays theoretically and practically, as well as designed and manufacturing performance models meant for use on spacecraft. For example, the Massachusetts Institute of Technology has developed a capable electrospray thruster using micro-fabricated parts, known as micro electro-mechanical systems (MEMS), and is scalable for specific thrust demands [25]. Though models featuring a high density of emitters may be more applicable to the

design that will eventually be used in space, there are still quite a few examples of colloid thrusters with a single or only a few emitters [15]. Topics about the exact nature of phenomenon during electrospray operation continue to be researched. For example, a study conducted at Konkuk University in Seoul about how multiple emitters cause interference with each other during operation showed the cone-jets on emitters at the edge of the array will form with a skewed axis relative to the emitter axis [26].

Perhaps the most notable example of electrosprays thrusters being used successfully in space is the ESA LISA Pathfinder mission, launched in December 2015 [9]. LISA Pathfinder's mission purpose was primarily a technology demonstration that would provide confidence in future missions using the same technologies. The spacecraft's drag-free dynamic control system (DCS) included colloid micro-Newton 
thrusters (CMNT) developed by Busek Co., Inc. The DCS was tasked with providing high precision control over spacecraft attitude while also compensating for the drag experienced by the spacecraft as it orbited Earth. For this task, CMNT provided 5-30 $\mu \mathrm{N}$ of thrust with better than $100 \mathrm{nN}$ of thrust accuracy. The thrusters operated for over 2400 hours and are the first demonstration of electrosprays in space.

Several companies have dedicated resources to developing performance electrospray thruster modules aimed at serving as propulsion for small satellite designs. Busek Co., Inc., works with their experience from the LISA Pathfinder mission to develop an electrospray module for use on CubeSat satellite form factors [17]. Another company, Accion Systems Inc., is also developing electrospray thrusters for use on small spacecraft. Their TILE design boasts modular packaging, allowing for multiple units to be placed side by side in order to achieve the level of thrust desired [18]. Performance models will typically feature densely packed emitter arrays to create large thrust densities, which can be contrast to designs utilized in research that contain smaller arrays or a singular emitter. 


\section{Chapter 3}

\section{DEMONSTRATION THRUSTER DESIGN AND TESTING}

The first goal of this thesis is to design and operate an electrospray thruster that would serve as a demonstration of the general principles of electrosprays. For this thruster, design choices were influenced most by the intention of making the thrusters operation and fundamental components highly visible. Predictions and analysis of the thruster's performance provided guidelines on specific values to choose for testing. The primary goal of testing was to prove the general concept of achieving emission and be able to visualize it thoroughly.

\subsection{Design Principles}

The basic principles that influenced the design of the Demonstration thruster are based more in qualitative understanding rather than any rigorous assessment of the thruster's performance. Because the thruster is operated in atmosphere, there are less analogs to be drawn between this design and those meant for use on spacecraft. Because of this, focus was given to highlight the basic concepts of the electrospray technology as applied to propulsion, even for those outside the field of astronautics or propulsion. Refer to figure 3.1 throughout the description of the design elements.

A single emitter was chosen as only one is necessary for exhibition of the core concepts of an electrospray thruster. For this emitter, an internally wetted capillary needle was chosen for its ability to accommodate the widest range of thruster orientations and propellants. At the sizes of needles that would be used for this thruster's purpose, there is a large variety of needles available. Externally wetted emitters would not work well with core goal of making the Demonstration thruster accessible, as the machining techniques required to fabricate this emitter type are more complex than necessary. A porous emitter would greatly increase complexity, both in the sense of thruster fabrication and testing procedure as the porous materials are more difficult to load and clean between tests, if they can be used again at all [6]. 


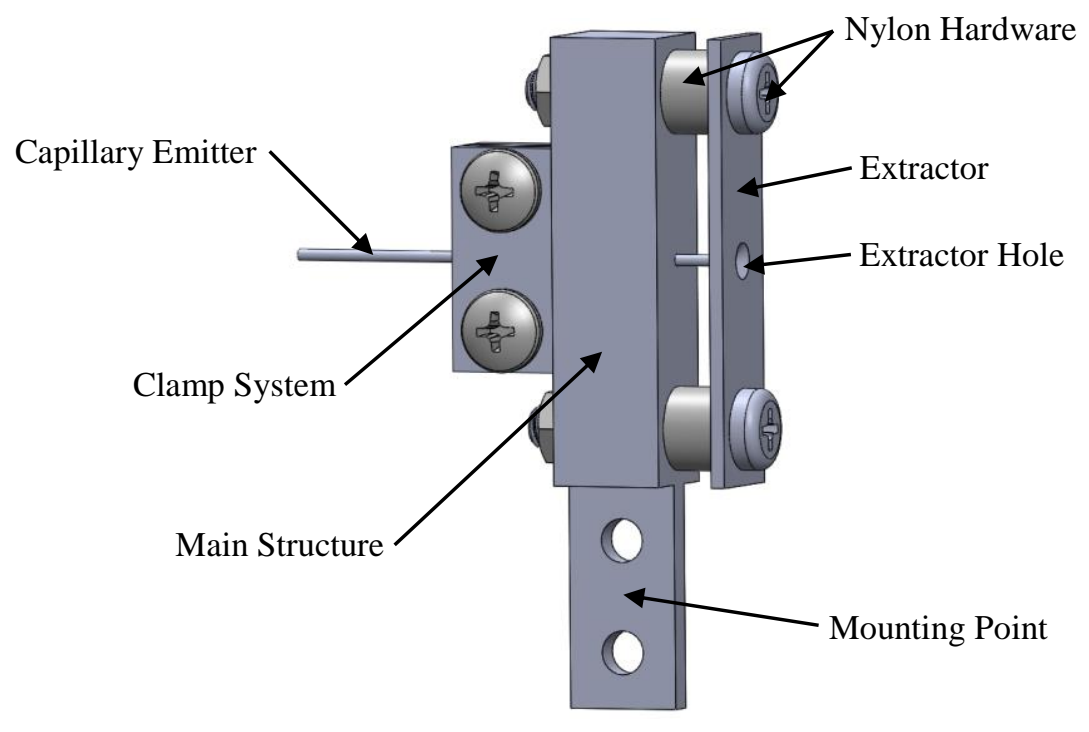

Figure 3.1: Assembled 3D Model of the Demonstration Thruster, Showing Major Design Elements.

The needle emitter is secured in its position by the use of metal clamps and silicone grip pads in order to provide sufficient friction without damaging the needle itself. This method was preferred over a permanent attachment to allow for the emitter to be able to be moved in relation to the extraction grid, allowing the user to choose this distance easily. This distance is important for the operation of the thruster, as will be discussed in section 3.1.2. This design element also allows the needle to be removed for replacement, easier cleaning, and storage as necessary.

An active propellant feed in the form of a syringe pump was chosen as the propellant delivery method, as opposed to attempting a passive method. This will allow more accurate control over flow rates and simplify propellant loading procedure, as the only preparation is to fill a syringe with propellant, rather than saturating a porous emitter or wetting a solid needle. The syringe is connected to the emitter through a section of flexible silicone tubing, providing a tight seal.

The Demonstration thruster's design includes only an extractor grid and not an accelerator grid for the sake of simplicity and reduced risk of arcing. Because this thruster design is meant purely as a source of visualization of the basic concepts of electrosprays applied to propulsion, the decision was made not to add unnecessary complexity. This thruster's testing will be conducted in atmospheric pressures, and so poses an increased risk of arcing when compared to adequate vacuum conditions. While the condition of arcing in the 
sense of raw distances and breakdown voltages can be designed around, the addition of propellant emissions during thruster operation can cause unexpected problems.

The chemical propellant chosen for the initial testing done in this thesis was acetone. This chemical is very common in laboratory settings, making it easy to acquire at reasonable purities, and carries minimal health hazards. Acetone's physical properties are satisfactory for the visualization nature of this testing; it is conductive, which of course allows emission to begin with, and has a low surface tension, promoting emission at lower electric field strengths, as is discussed in section 3.1.2. One downside to this propellant is its high vapor pressure, which enables the propellant to evaporate at atmospheric pressures, but this can be compensated by the flowrate chosen on the syringe pump. Other propellants could be used for this testing, such as glycerol, however this would require higher voltages to achieve emission and increase the risk of arcing. Acetone provided the great properties of availability, ease of emission, and low-risk.

\subsubsection{Technical Specifications}

Many of the major dimensions of this design are chosen because of the nominal dimensions of the stock materials utilized. Since these dimensions have little impact of the performance of the thruster as a whole, a simplified fabrication process is desirable. All machining operations and tolerances necessary are able to be performed on a common mill with standard tooling. Other than enabling the proper function of the thruster, the design was tailored to promote easy assembly and handling, and durability to prevent warping.

A technical drawing of the assembled thruster is shown in Figure 3.2 with major dimensions provided. Many of the major dimensions were retained from the stock material the thruster parts were machined from, such as the major depth of 0.5 inches and the width of 1 inch of the main thruster structure. This width was necessary to include enough surface area for the clamp mechanism to grip the emitter needle, as well as for reduced error in the angular alignment of the emitter itself. The depth of 0.5 inches allows for the thruster to have a reasonably slim profile, while also having significant durability. The overall height was made as small as possible while including enough space for the various components to be assembled with common hand tools. A protrusion of material was necessary underneath the main thruster to allow mounting on the testing support structure. 


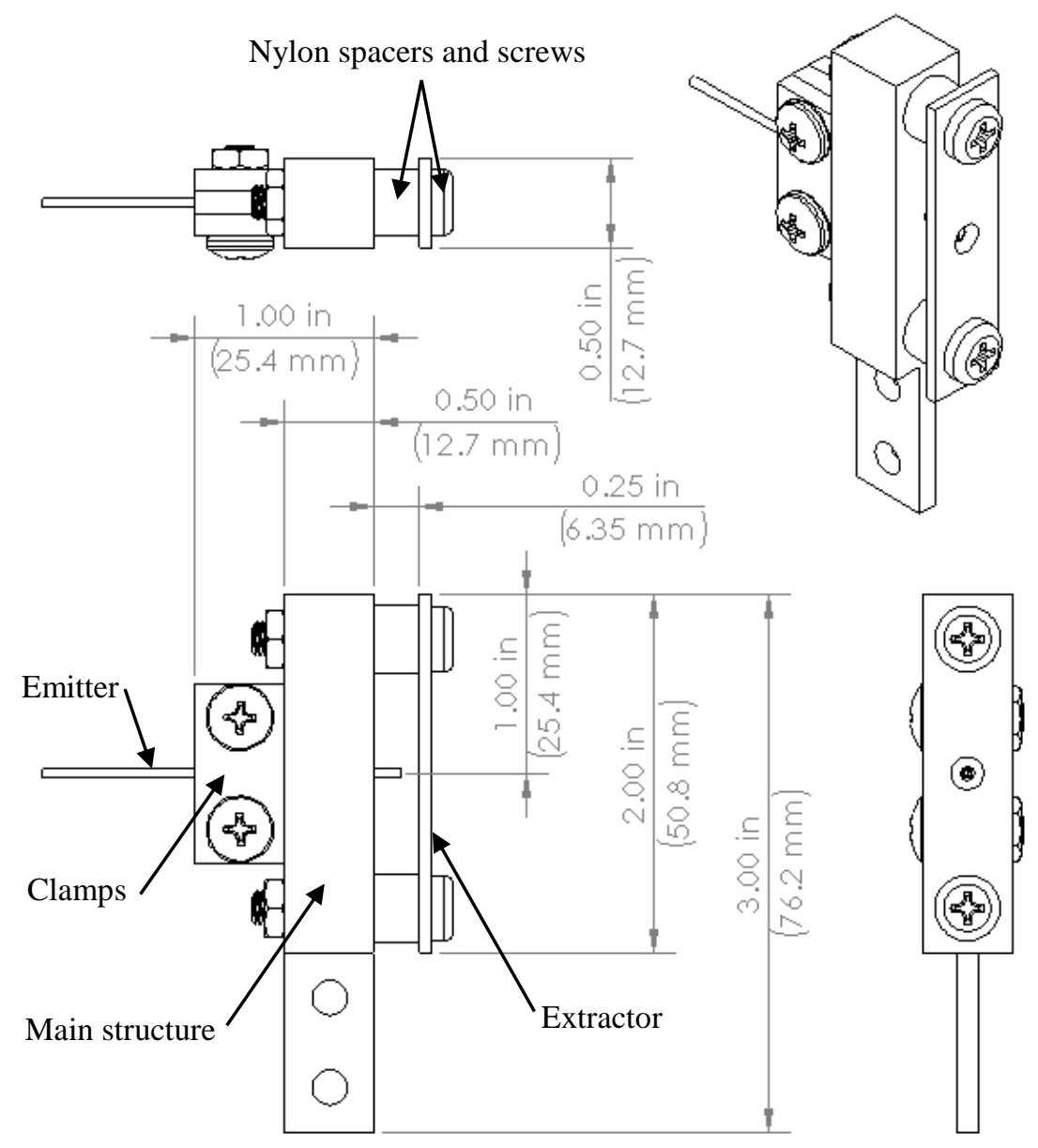

Figure 3.2: Three View Drawing with Major Dimensions and Isometric View of the Assembled Demonstration Thruster.

The main structure, clamps, and extractor are machined from aluminum stock for its machinability, conductivity, chemical resistances, and cost effectiveness. The fasteners are all stainless steel with the exception of the electrically isolating nylon spacers and screws, meant to separate the main structure and extractor. Nylon was chosen for its insulating properties foremost, but also its cost effectiveness and availability as machine screws.

The emitter was purchased as a common 18 gauge stainless steel blunt tip dispensing needle. Stainless steel is very chemical resistant and conductive enough for its purpose. Since no major machining was necessary other than cutting the needle to length, machinability was not a factor. These needles have an inner diameter of 0.038 inches, which is large from the perspective modern electrosprays, but is suitable for colloids. As the propellant flows from the emitter tip, the size choice provides a very clear visual the emission 
modes the thruster is operating in and the formation of cone-jets. The hole the emitter sits in through the main structure was machined to prevent angular misalignment as much as possible while maintaining some come clearance for assembly. With a hole 0.052 inches in diameter and an assumed max tolerance of plus 0.002 inches, the most the emitter can be angled relative to main structure is 0.458 degrees. This is more than acceptable for this design, as performance is not a particular concern, and the alignment through this method can never be great enough to adversely affect emission interception on the extractor.

The clamp design for securing the emitter allows for the easy customization of the distance between the tip of the emitter and the extractor. This distance is crucial for proper operation of the thruster, and any variation with change how the thruster emits propellant. Precision machined spacers were used to set the distance for the emitter. Taking into account the tolerance of the spacers and the general ability to manually align the distance, this distance could be aligned with an accuracy of about 0.005 inches, which is acceptable for the purpose of this thruster.

Ideally, the extractor thickness should be as thin as possible to reduce interception, but the grid does still need structural stability to prevent misalignment. For this thruster design, the extractor is supported by the two nylon spacers that are a reasonable distance away, so a thickness of 0.0625 inches was chosen to maintain stiffness while mounted, as well as make use of common stock dimensions. Figure 3.3 shows a close up cross section view of the extractor and emitter alignment. The extractor hole that allows the emitted propellant to be expelled as thrust features a countersunk outward face in order to reduce interception of the emission plume, which reduces performance. A 45 degree countersink was created and reduces the apparent thickness of the emitter to 0.010 inches, which is thick enough to prevent excessive marring of the hole when handling and cleaning, but clearly much thinner than the major thickness of the extractor. The distance between the emitter and extractor given in the drawing is the value used during testing, and is explained in the section 3.1.2. 


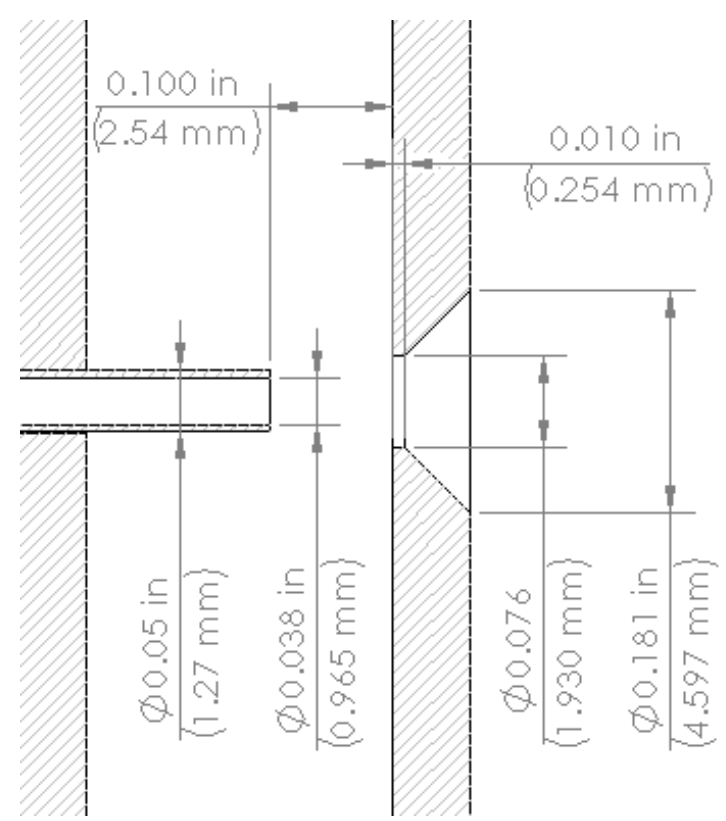

Figure 3.3: Close-Up, Cross Section View of Emitter and Extractor Alignment on the Demonstration Thruster with Major Dimensions.

\subsubsection{Thruster Performance Expectations}

For the purpose of this thesis, the depth of the initial characterization of the Demonstration thruster is limited to the prediction of successful emission and a voltage breakdown analysis to avoid arcing between thruster components or the surrounding area. Additionally, a simple two-dimensional analysis of the electric field between the emitters and the extractor plate to visualize the electric field formation. This provides a basic understanding of how the propellant will respond to the electric field created, and is sufficient to be able achieve emission.

Applying equations 2.8 and 2.9 for a prediction of onset voltage to the Demonstration thruster system with a varying extractor distance from the emitter, the trend seen in Figure 3.4 is achieved. For the 18 gauge needle used as the emitter, an outer radius of $0.025(0.635 \mathrm{~mm})$ inches is used, assuming the propellant will wet the flat surface of the blunt needle, providing a worst case scenario for emission. The surface tension of acetone is $24 \mathrm{mN} \mathrm{m}^{-1}$ [27]. The distance between the emitters is bounded by values that were deemed reasonable from a perspective of understanding how the propellant will extend past the emitter tip during emission. 


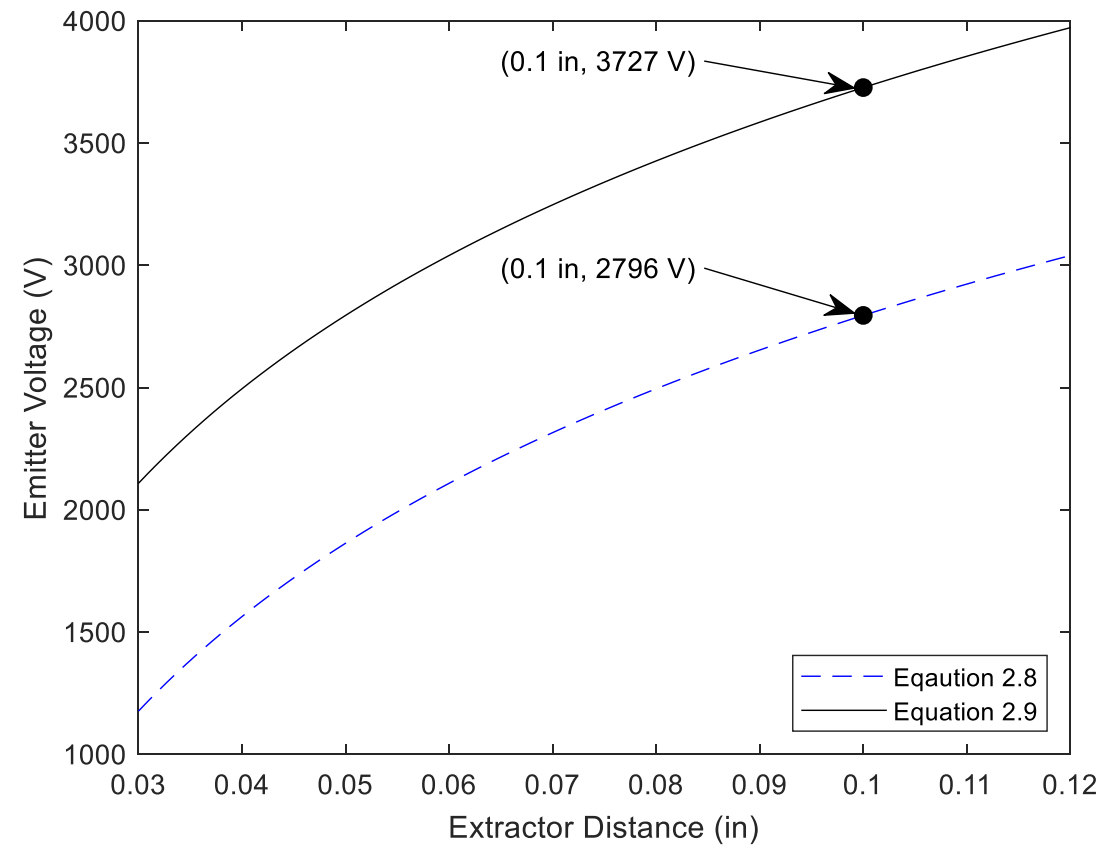

Figure 3.4: Onset Voltage Trend for the Demonstration Thruster Across a Range of Distances Between the Emitter and Extractor.

To ensure safe operation in atmospheric pressures, where electrical breakdown is often easier than in the vacuum pressures electrosprays are typically operated in, an analysis of the breakdown voltage threshold using equation 2.10 was compared to the expected operational voltages. The pressure $p$ is constant atmospheric pressure at 760 Torr, and the distance between the electrodes $d$ is varied across a large range of values. For the conditions in standard air, the constants of $A$ and $B$ are found to be $11.25 \mathrm{~Pa}^{-1} \mathrm{~m}^{-1}$ and 273.75 $\mathrm{V} \mathrm{Pa}^{-1} \mathrm{~m}^{-1}$, respectively, and the coefficient of secondary electron emission $\gamma_{s e}$ is 0.01 [21]. Figure 3.5 shows the resulting Paschen breakdown curve, alongside the practical limits that would be seen during testing with the demonstration thruster. The breakdown voltage intersects the maximum voltage of $3700 \mathrm{~V}$ that would be applied to the thruster at an electrode distance of about 0.026 inches. From this information, it was decided to set the emitter to extractor distance on the Demonstration thruster to 0.100 inches ( $2.54 \mathrm{~mm}$ ), providing a large factor of safety even when expecting the propellant's droplets or cone-jet to effectively reduce this gap distance. The only area that arcing can occur with this thruster is the smallest electrode distance, which is the spacing between the emitter and extractor, because the atmospheric pressure does not change significantly and as the electrode distance increases, the voltage required for breakdown to occur always increases. 


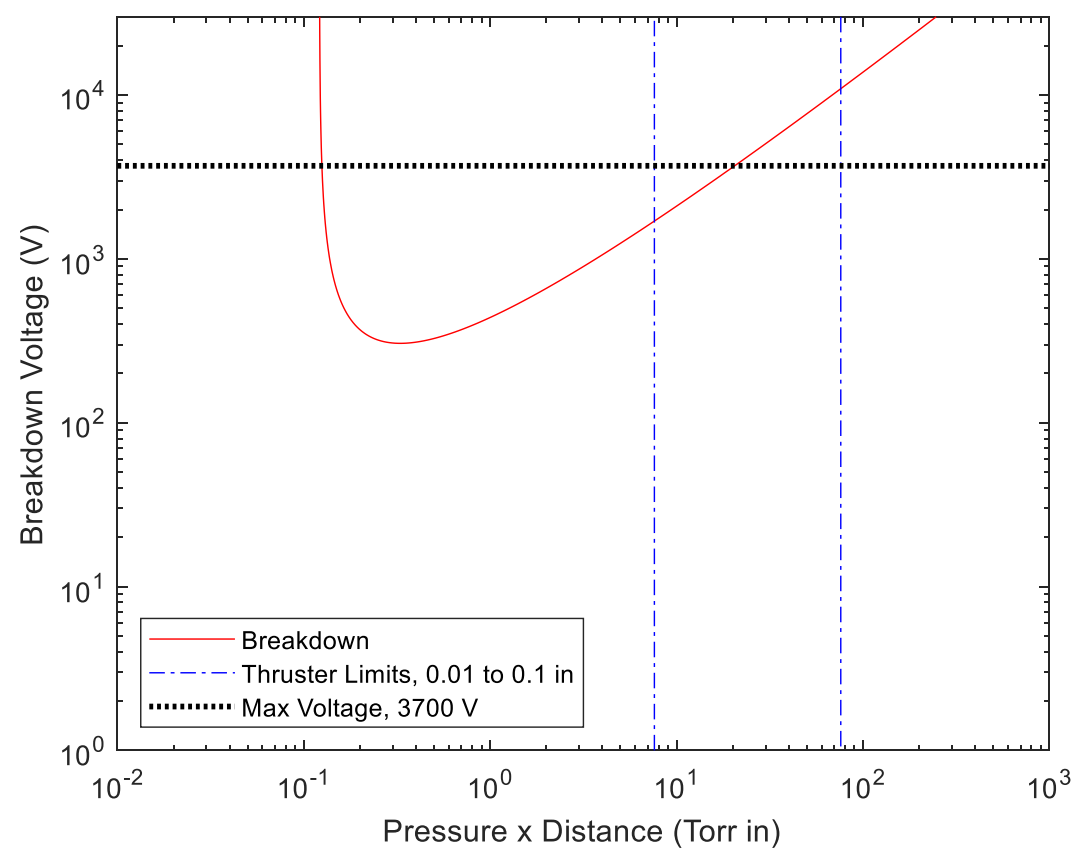

Figure 3.5: Calculated Paschen Breakdown Curve in Air with Relevant Values for the Demonstration Thruster.

In an effort to have a better visualization of the formation of the electric field lines when voltage is applied to this thruster, a brief analysis was completed in the computational fluid dynamics program, CFDACE [28]. This program has the ability to map electric field strengths on a mesh. A two dimensional simplified cross-section representation of the Demonstration thruster is input as a mesh and voltage is applied for a single scenario. Figure 3.6 shows a close up view of the electric field strength vectors near the emitter tip and the extractor. This analysis is validated for accuracy using a simple mesh of two biased plates separated by a distance. Additional details of this CFD analysis can be found in appendix A. 


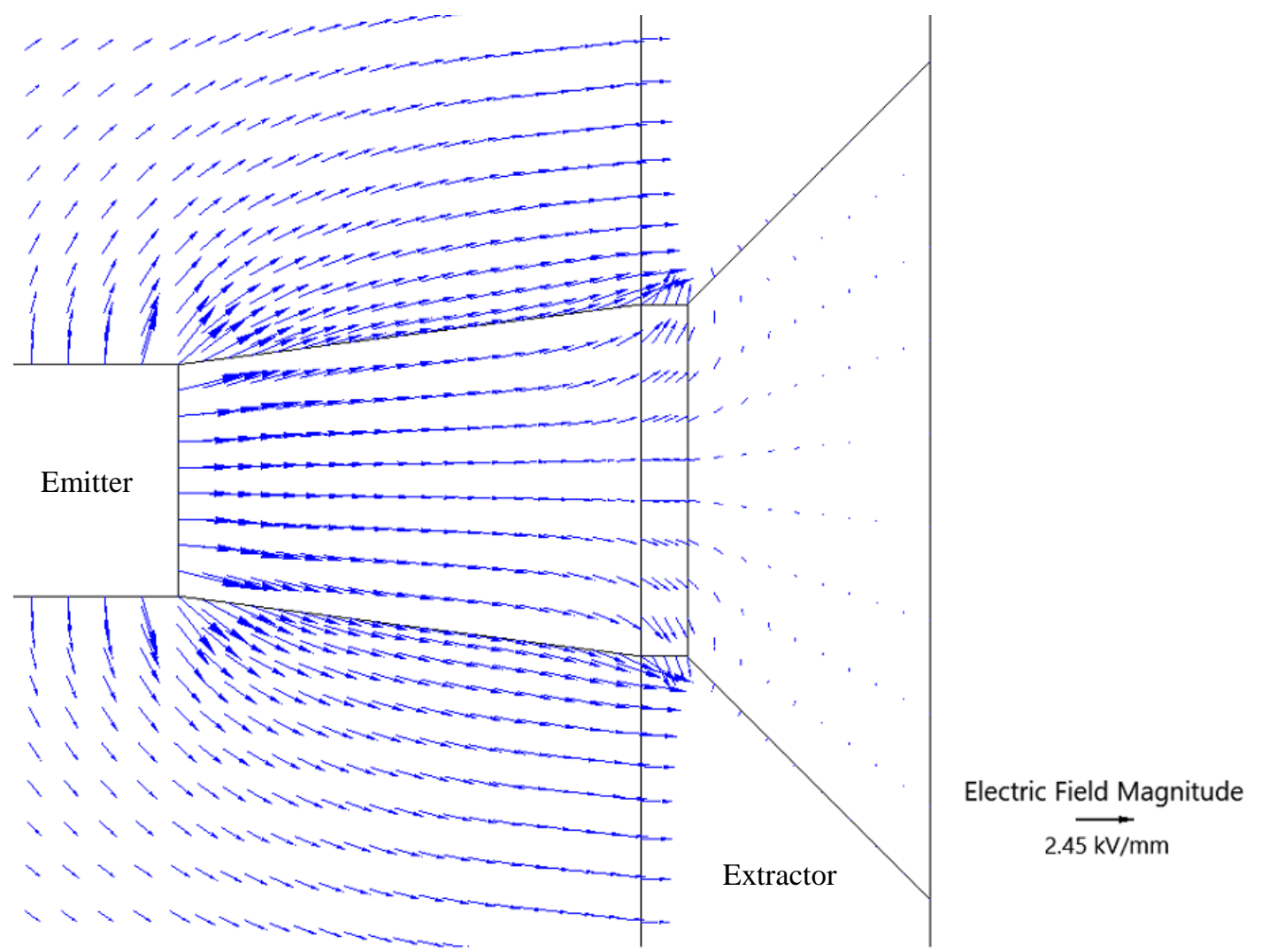

Figure 3.6: Vector Plot of the Electric Field Strength near the Demonstration Thruster Emitter and Extractor.

For the single scenario computed, an emitter to extractor distance of 0.100 inches $(2.54 \mathrm{~mm})$, and a charged extractor bias of positive $2700 \mathrm{~V}$ was used. The emitter tip shape does not take into account the formation of propellant into a Taylor cone or any other shape, and just assumes a solid blunt tip. This analysis is purely for visualization purposes, and had no impact on the thruster design or operation. On the surface of the emitter tip, the electric field vectors have a significant vertical component, and have a greatest total magnitude near the corners. The electric field is relatively horizontal between the two surfaces and decays as expected. Past the inner face of the extractor and through the hole, the electric field curls back towards the extractor. Though the field strength in these areas is much less than the previous areas where horizontal acceleration will occur, if there are any particles with low velocity at this point, there is a higher likelihood that they will be attracted to extractor and not contribute to thrust. If the propellant builds up and accumulates there, partial or full blockage of the extractor hole may occur causing increased interception rates, potentially leading to thruster failure. 


\subsection{Experimental Setup and Diagnostics}

The testing completed for this thesis was a simple verification that the thrusters are emitting, accomplished by direct measurement of the current imparted by the emissions on a collector plate. Figure 3.7 shows a simple diagram of the electrical paths required for operating the thruster. Current data was collected from both the collector plate and the extractor grid, giving a representation of thruster performance by comparing the amount of propellant emissions intercepted by the extractor to the amount of propellant emissions that escapes the thruster completely. For this testing, the power supply was connected to charge the emitters, which are electrically connected to the main thruster body. This allows the intercepted current on the extractor to be measured without the need for any high voltage current measurement system.

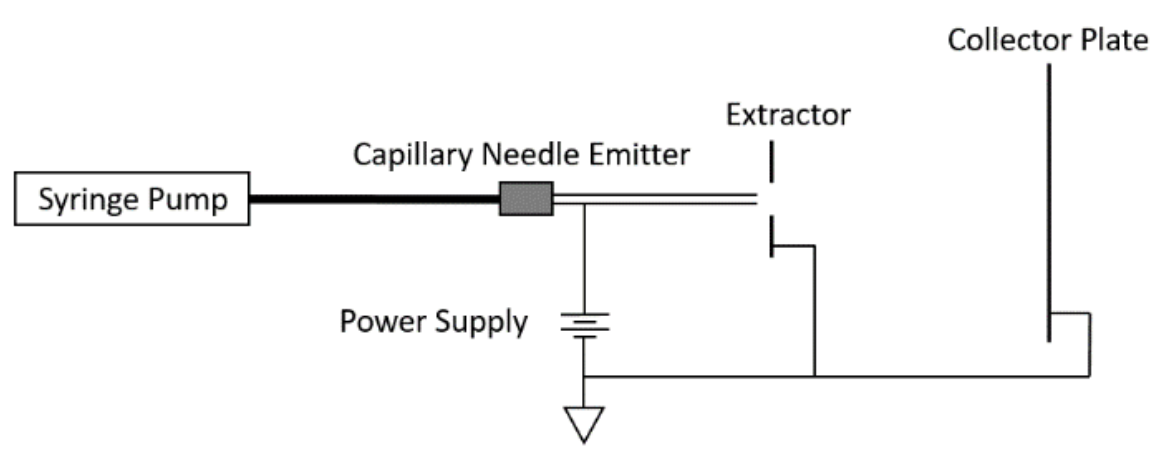

Figure 3.7: Simplified Electrical Diagram of the Demonstration Thruster.

The power supply and pico-ammeter are rack-mounted and located nearby. For the testing conducted in this thesis, a Glassman High Voltage Inc. EL Series power supply, Model EL10P04.0 was used as the source of voltage to charge the components of the thruster, providing up to positive $10 \mathrm{kV}$ at up to 4 mA current. A Keithley Model 6487 pico-ammeter was used to directly measure current with femto-Amp readouts. Additionally, a Keysight Infiniivison Oscilloscope Model DSOX2004A was used to more accurately measure the voltage being applied to the thruster, as the Glassman power supply used is analog only. The syringe used for the active propellant flow is a New Era Pump Systems NE-300 Just Infusion Syringe Pump. The block diagram in figure 3.8 shows a picture of the experimental setup used in semiaccurate relative positions to the physical setup. 


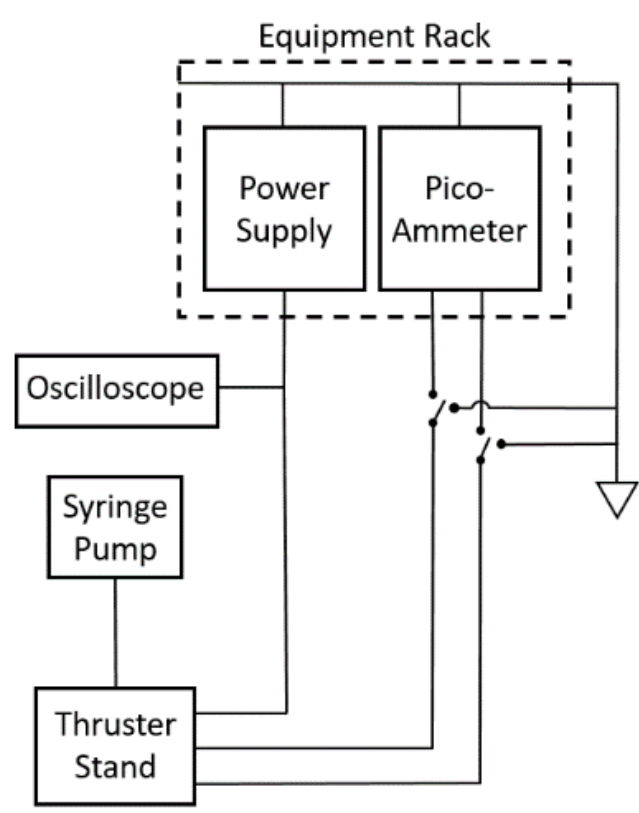

\section{Figure 3.8: Block Diagram of Experimental Testing Setup of the Demonstration Thruster Including Electrical Equipment.}

The power supply is connected to the thruster using a series of RG-8U cabling and 22 AWG high voltage wiring, and the extractor grid and collector plate are connected using standard BNC cables and 22 AWG high voltage wiring. Because the pico-ammeter can only measure one current at a time and only one pico-ammeter was available during this testing, when either the extractor or the collector is connected, the other is connected directly to ground. The connections are switched when necessary to get the appropriate data.

The experimental setup was designed to be contained on a single platform to promote easy handling and consistent use; all major thruster components are located or secured on a single piece of plywood. A picture of the test-ready setup is given in figure 3.9. The thruster was mounted to the board using polycarbonate strips to isolate the main thruster structure conductively. The collector plate is similarly isolated from its mounting supports using silicone strips and nylon screws. For this testing, a syringe with an internal volume of $3 \mathrm{~mL}$ was used. This syringe was connected to the emitter using a length of silicone tubing. A protective box made of polycarbonate surrounds the thruster and collector plate to prevent any stray propellant emissions from leaving the test area, and to prevent accidental contact of the charged thruster components by the operators or observers. The mounted thruster assembly is shown in closer detail in figure 3.10. The electrical connections to the thruster parts are made using tin-coated copper ring terminals and 
secured by machine screws. A small PTFE plate was secured with tape on the opposite of the thruster as an opaque backdrop to enhance the quality of images and video taken of the thruster's operation. Images and video were captured using the camera on a Samsung Galaxy S8 smartphone.

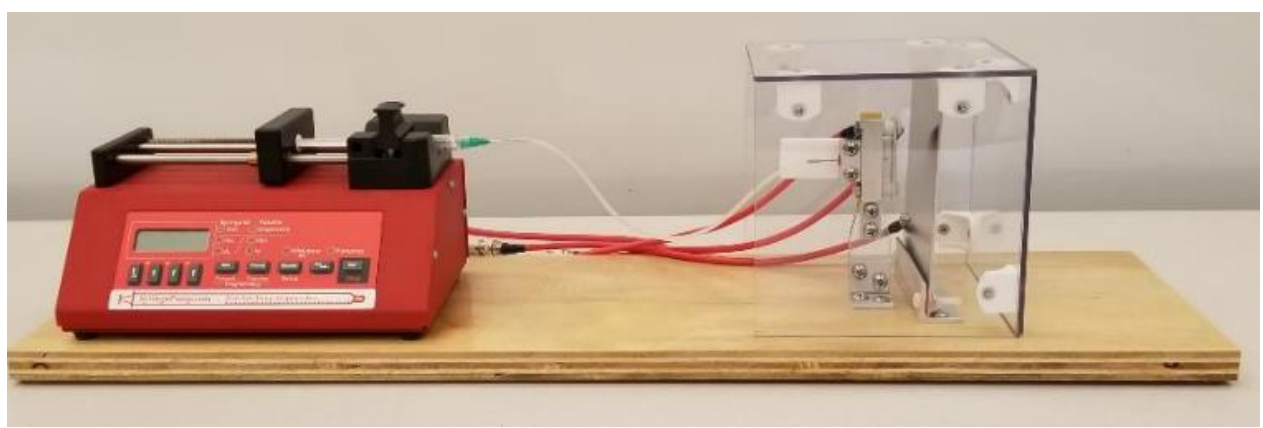

Figure 3.9: Demonstration Thruster in Its Testing Apparatus with the Syringe Pump.

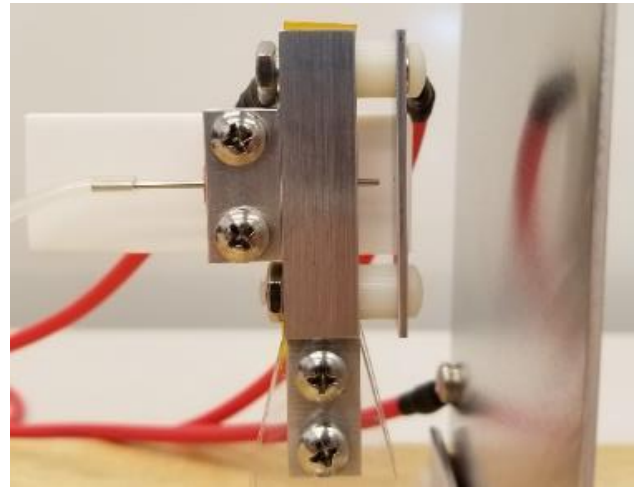

Figure 3.10: Close-Up of the Demonstration Thruster in Its Testing Apparatus Next to the Collection Plate.

\subsection{Testing and Results}

The justifications coming from the theoretical analysis served to create a general testing outline for the demonstration thruster, in terms of the minimum and maximum voltages applied. A testing procedure was planned and executed to gather numerical measurements and visual pictures and videos to confirm the successful operation of the thruster, where emission currents are measured on the collection surfaces. Data are recorded at a sample rate of at least $8 \mathrm{~Hz}$ through a LabView virtual interface that communicates with the pico-ammeter and the oscilloscope. 


\subsubsection{Basic Testing Procedure}

The basic testing preparations and operation procedure is described here. A more detail procedure including assembly is provided in appendix B. Once the testing apparatus is correctly assembled and all the electrical connections are made, the diagnostics equipment is given at least 30 minutes to warm up before testing can begin, as recommended by the relevant user's manuals for better performance. Before testing with propellant (referred to as "live" testing throughout this thesis), at least one dry run for each the extractor and the collector would be completed to ensure that all the electronics are working properly and the system is responding to voltage consistently. The old age of the power supply can cause some irregularities over time, and the voltage steps for testing may need to be adjusted accordingly. Due to the power supply being operated through a physical dial, there is significant human error in setting the voltage. Additionally, the particular power supply used in this experimentation was not accurately calibrated to the position of the dial, and so the actual voltage supplied increases non-uniformly relative to the setting on the dial. Only the real voltages are reported.

Dry testing consisted of a voltage sweep from $1600 \mathrm{~V}$ to $3700 \mathrm{~V}$ in 17 steps with 10 seconds spent at each voltage on rise and fall of the sweep. This is beyond the range for the live testing, and is to establish an understanding the background noise in the data collection at each voltage. For live testing, a voltage sweep from $2100 \mathrm{~V}$ to $3700 \mathrm{~V}$ in 11 steps with 30 seconds at each voltage. At least 30 seconds was spent at $0 \mathrm{~V}$ before and after the voltage sweep to establish an offset value for the pico-ammeter, in the event that needed to be accounted for in data processing.

Testing was initiated by pumping acetone propellant to the tip of the emitter and balancing the flow rate with the rate of evaporation to form a consistently sized bead at the very tip of the emitter needle. The evaporation rate can vary greatly based on small differences in atmospheric conditions, the pumping rates necessary to achieve a constant propellant bead varied from 8 to $11 \mu \mathrm{L} / \mathrm{min}$, though this effect is not measured explicitly. These flow rates were kept constant during the entire testing sweep. Before starting data collection, video capture was initiated. Between voltage sweeps, the pump was turned off and the BNC cables leading to the pico-ammeter were switched to gather data on the other collection surface, either the collector or the extractor. When the testing is concluded, any pictures of testing that are necessary are taken, and the parts 
are cleaned as necessary. The thruster components are cleaned between testing sessions, which typically included multiple voltage sweeps.

\subsubsection{Visual Thruster Operation}

Video recordings and images of thruster operation and physical results were taken for all live testing runs. Focus is given to the cone-jet formation at the tip of the emitter. Note that the pictures are shown with the emitter facing upwards, but the thruster is actually emitting horizontally. In the picture's orientation, the right direction is downward in reality.

For emitter voltages less than about $2700 \mathrm{~V}$ on the testing sweeps with the collector hooked up to the pico-ammeter, the thruster emitted in the dripping mode, oscillating between a blunted bead of propellant and a short emission period featuring and unsteady Taylor cone. This progression over a period of 10 seconds can be seen in figure 3.11. Emission appears to occur for about a second, before enough propellant is removed to increase the distance to the extractor and stop emission altogether. At voltages just slightly higher than this at $2810 \mathrm{~V}$, a stable cone-jet is formed and emission is completely steady over the entirety of operation. Figure 3.12 shows emission over a similar time period of six seconds. It is worth noting that the propellant cone is offset in the downward direction. This is likely due to a combination of some misalignment between the axis of the emitter and the axis of the extractor hole and the gravity force acting on the propellant as it cantilevers past the end of the emitter.

The steady cone-jet continues into the high voltages up $3700 \mathrm{~V}$, but begins to experience extreme distortion of the emission cone. The deviation as voltage is increased can be seen in figure 3.13. The increasing angle in the pointing direction of the cone will undoubtedly increase the propellant interception

rates onto the extractor, reducing performance. This prediction is unconfirmed with the level of testing completed for this thruster, but is a safe assumption using the understanding the formation of the electric field lines gained from the computational analysis done in section 3.1.2. 


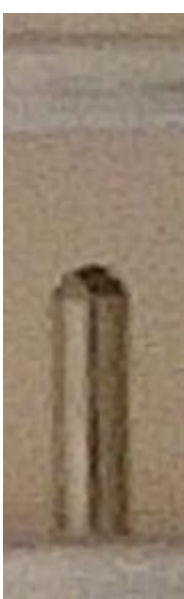

(a)

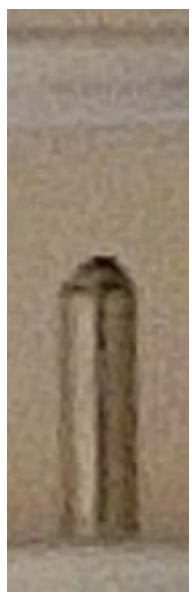

(b)

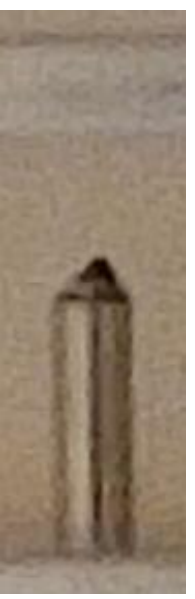

(c)

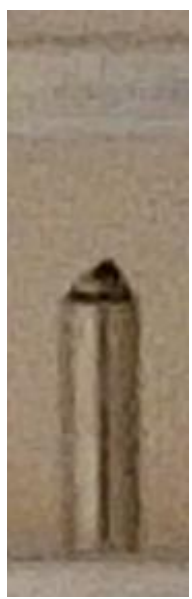

(d)

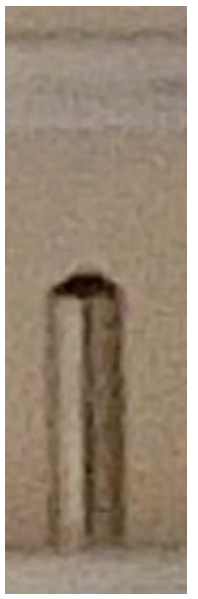

(e)

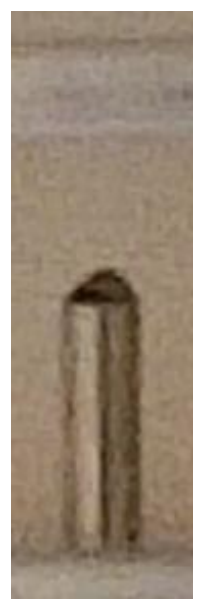

(f)

Figure 3.11: Progression Over 10 Seconds of the Demonstration Thruster Emitting in the Unsteady Cone-Jet Mode with the Emitter Charged to 2670 V: Approximately at Relative Times 0 s (a), 3 s (b), $4 \mathrm{~s}(\mathrm{c}), 5 \mathrm{~s}(\mathrm{~d}), 6 \mathrm{~s}(\mathrm{e})$, and $9 \mathrm{~s}(\mathrm{f})$.

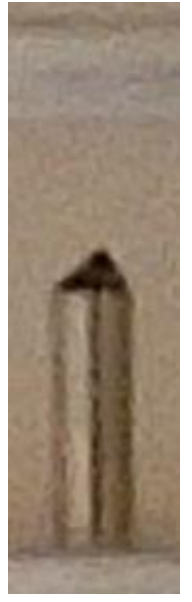

(a)

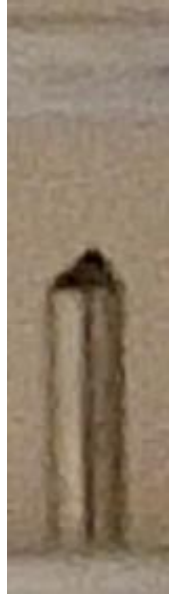

(b)

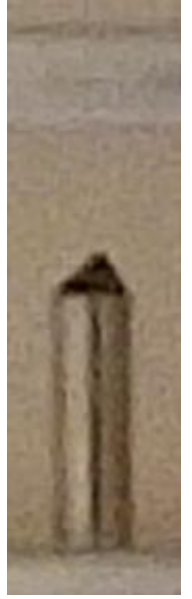

(c)

Figure 3.12: Progression Over 6 Seconds of the Demonstration Thruster Emitting in the Steady ConeJet Mode with the Emitter Charged to 2810 V: Approximately at Relative Times 0 s (a), 3 s (b), and 6 $\mathbf{s}(\mathbf{c})$.

To speak to the differences of the collector sweeps and the extractor sweeps again, figure 3.14 shows the disparity of operation clearly. With the emitters at the same voltage, the two scenarios experience vastly different emission qualities. At $3750 \mathrm{~V}$ for the collector current measurement setup, the propellant experienced an extreme amount of offset emission and is operating in a steady cone-jet emission mode. At a similar voltage of $3730 \mathrm{~V}$ for the extractor current measurement setup, the thruster is not emitting in a steady cone-jet at this point. This is a stark contrast to emissions from the tests that the collector current is measured, where steady cone-jets are formed at voltages as low as $2800 \mathrm{~V}$. 


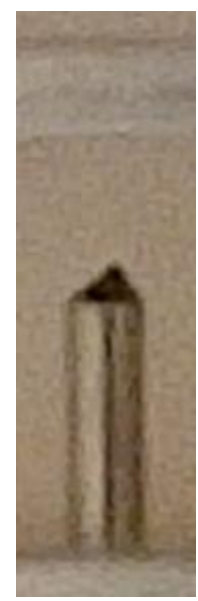

(a)

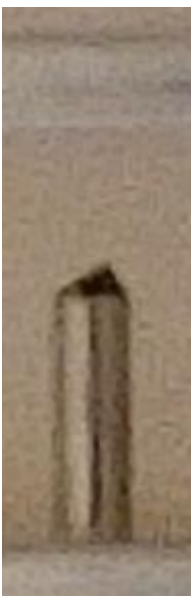

(b)

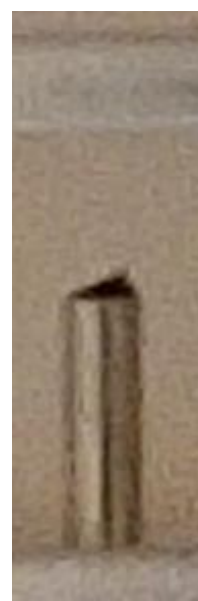

(c)

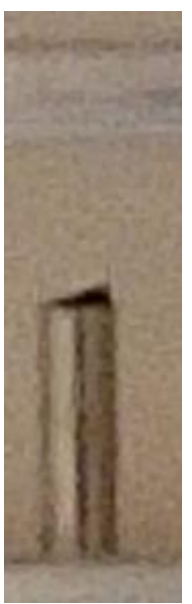

(d)

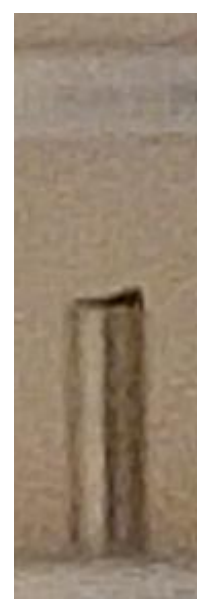

(e)

Figure 3.13: Steady Cone-Jet Formation of the Demonstration Thruster with the Emitter Charged to Different Voltages: at $2950 \mathrm{~V}$ (a), $3160 \mathrm{~V}$ (b), $3340 \mathrm{~V}$ (c), $3550 \mathrm{~V}$ (d), and $3750 \mathrm{~V}$ (e).

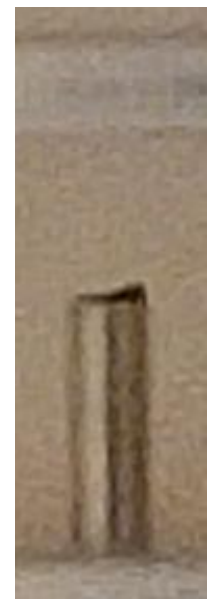

(a)

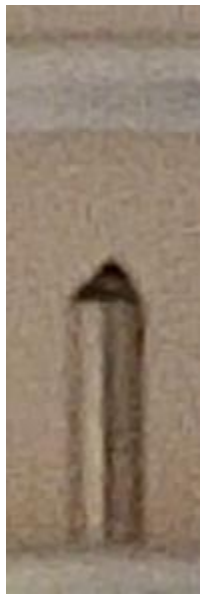

(b)

Figure 3.14: Comparison of the Demonstration Thruster's Emission Quality Between Tests Where Current is Collected from the Collector Plate (a) Versus from the Extractor (b).

As more proof of successful emission, an image of the collector plate wet with propellant can be seen in figure 3.15. There is a clearly discolored area where the propellant has landed on the aluminum. Though much of the acetone has evaporated by the time this photo was taken, the amount left and the residue remaining makes it clear how the emission plume expands as it leaves from the emitter and then past the extractor. This relates to the beam divergence of the emission, which reduces overall performance as the propellant gains velocity in the direction perpendicular to the thrust direction, and so does not create thrust. On a spacecraft, this divergence may endanger nearby components if propellant is deposited on them, and so must be designed around as the divergence is inescapable. No estimation of the divergence angle was made 
due to the inconsistency of the propellant deposition on the collection plate, caused by varying length of testing and evaporation of the propellant.

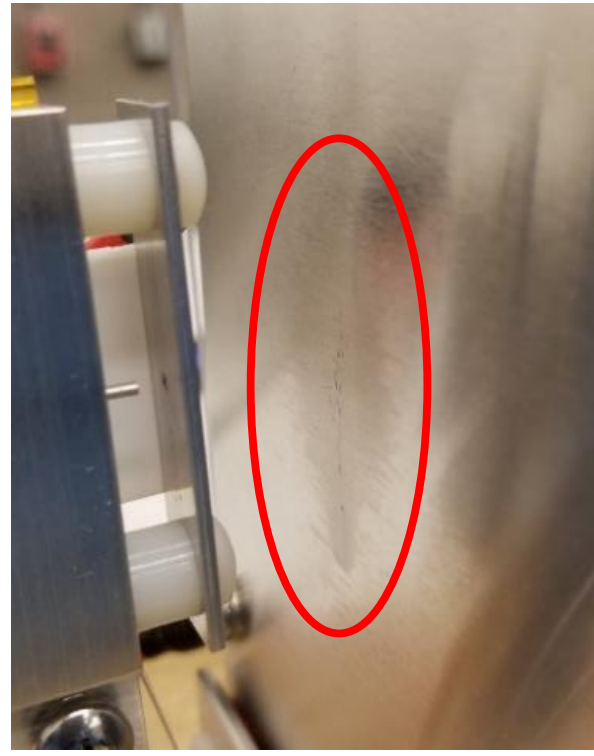

Figure 3.15: Typical Pattern of Propellant Deposited on the Collector Plate After Operating the Demonstration Thruster.

\subsubsection{Data Results and Discussion}

The testing of the Demonstration thruster consisted of five dry voltage sweeps and five live voltage sweeps per collection surface. Data was collected on all, and video was collected on all of the live tests. As stated before, the testing of this thruster is not meant for any rigorous analysis. The visual and quantitative confirmation that emission is being achieved is sufficient. Furthermore, there was a phenomenon occurring during operation where the charge of the emission plume would completely or partially dissipate before reaching the collection surface, and so would not consistently impart a measurable current. This phenomenon and other errors will be discussed later.

Raw data collected from one example of a dry run with the emitter voltage and collector current being measured over time is shown in figure 3.16. It is clear that even at the distance of the collector from the main structure, there is significant noise created by the high voltage. From a baseline noise on the order of $1 \times 10^{-11} \mathrm{~A}$ with the voltage at $0 \mathrm{~V}$ to as high as $1 \times 10^{-9} \mathrm{~A}$ at $3700 \mathrm{~V}$. The large spikes seen at the beginning and end of the voltage sweep are caused by the quickly changing voltage as the dial is turned. The plots in 
figure 3.17 show an example of a dry run with the extractor connected to the pico-ammeter. A similar trend is seen here, where the noise increases with voltage, as is the large spikes at the beginning and end. There is a significant difference in the magnitude of the noise however, due to the extractor's proximity to the charge emitter and main structure. A similar baseline noise at $0 \mathrm{~V}$ on the order of $1 \times 10^{-11} \mathrm{~A}$ occurs, but at higher voltages the noise can be as large as $3 \times 10^{-9} \mathrm{~A}$. Consistently, the largest spikes in current correspond to the transient periods of changing voltage, as can be seen most clearly with the extractor dry run in figure 3.17 . Note that the currents measured here are not related to noise present in the measurement equipment, but is in fact the real current measured due to the high voltage causing charge to be imparted on the measured collection surface through the air.
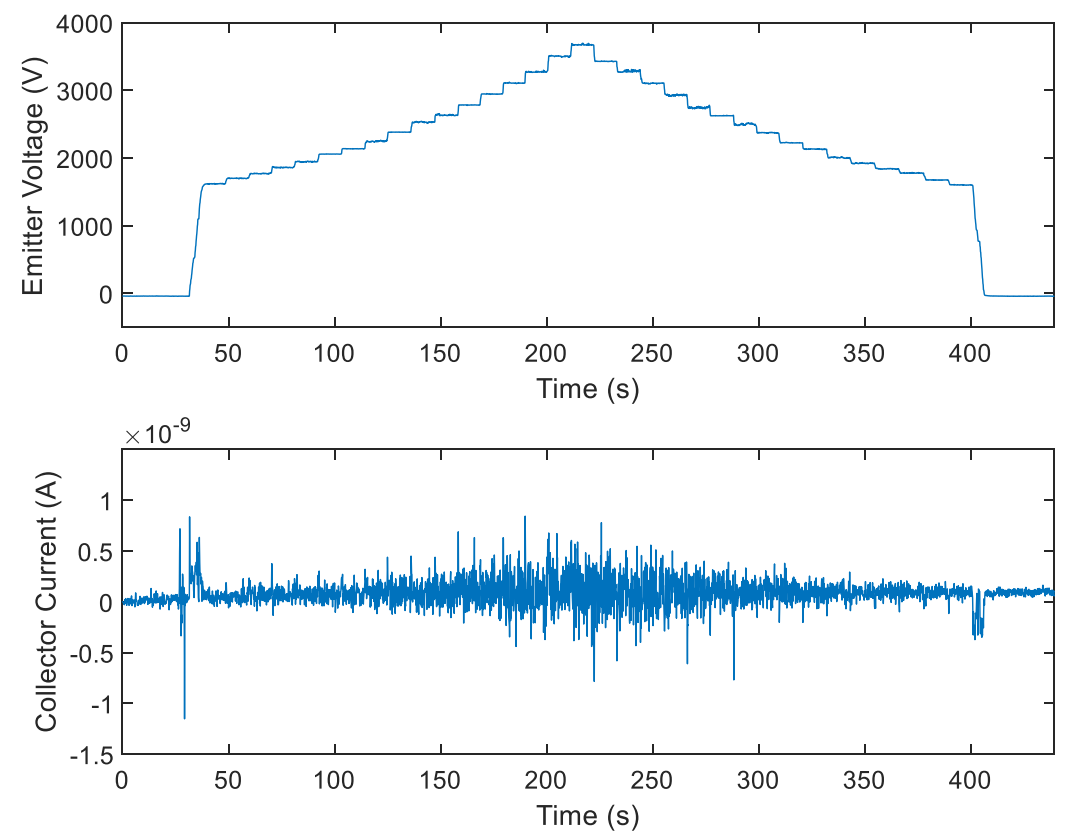

Figure 3.16: Typical Current Data from Collector Plate on the Demonstration Thruster During a Dry Voltage Sweep.

This background profile was consistent throughout all of the testing done on the Demonstration thruster. The envelope of current measurements grew with increasing voltage. At the maximum voltage of $3700 \mathrm{~V}$ across all of the testing, the current magnitudes measured on the collector plate were within an envelope of $8.5 \times 10^{-10} \mathrm{~A}$, while the current magnitudes measured on the extractor did not exceed $3 \times 10^{-9} \mathrm{~A}$. 

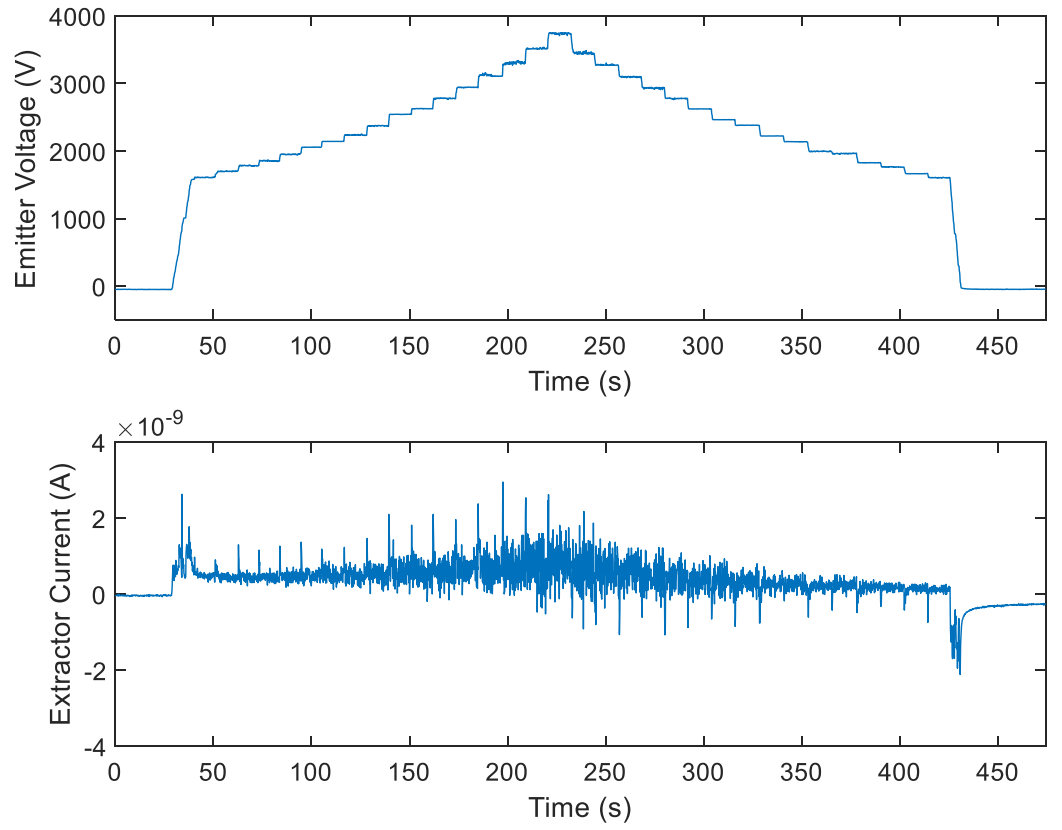

Figure 3.17: Typical Current Data from Extractor on the Demonstration Thruster During a Dry Voltage Sweep.

Figure 3.18 shows the raw data for a live test voltage sweep measuring the current on the collector. It can be immediately seen that there are current spikes that are distinctly larger than the background noise seen from the dry runs, confirming that some charged propellant emissions are reaching the collector plate. Peaks as high as $3.8 \times 10^{-9} \mathrm{~A}$ are seen for this particular example, and are larger than the noise generated from the large changes in voltage of starting and ending the voltage sweep. It may be worth noting that this is a positive current, which is expected given the direction of the electric field created by charging the emitters to positive voltage and grounding the extractor. These peaks are only detected at the lower voltages of the sweep, and essentially no response at the higher voltages differs from the noise profile observed with the dry sweep. The large current spikes are due to the thruster operating the dripping mode at these voltages. When the propellant is emitting during a dripping emission mode, there is a relatively large amount of propellant expelled compared to a steady cone-jet formation. At higher voltages starting at about $2670 \mathrm{~V}$ (around the 200 second mark) for this particular example, the propellant at the emitter tip forms a stable cone-jet, and so a consistent fine mist of particles are emitted rather than periodic large droplets. The finer mist of is likely more susceptible to losing its charge as it travels the distance to the collector plate, as it is interacting with large amounts of air molecules throughout its movement. 

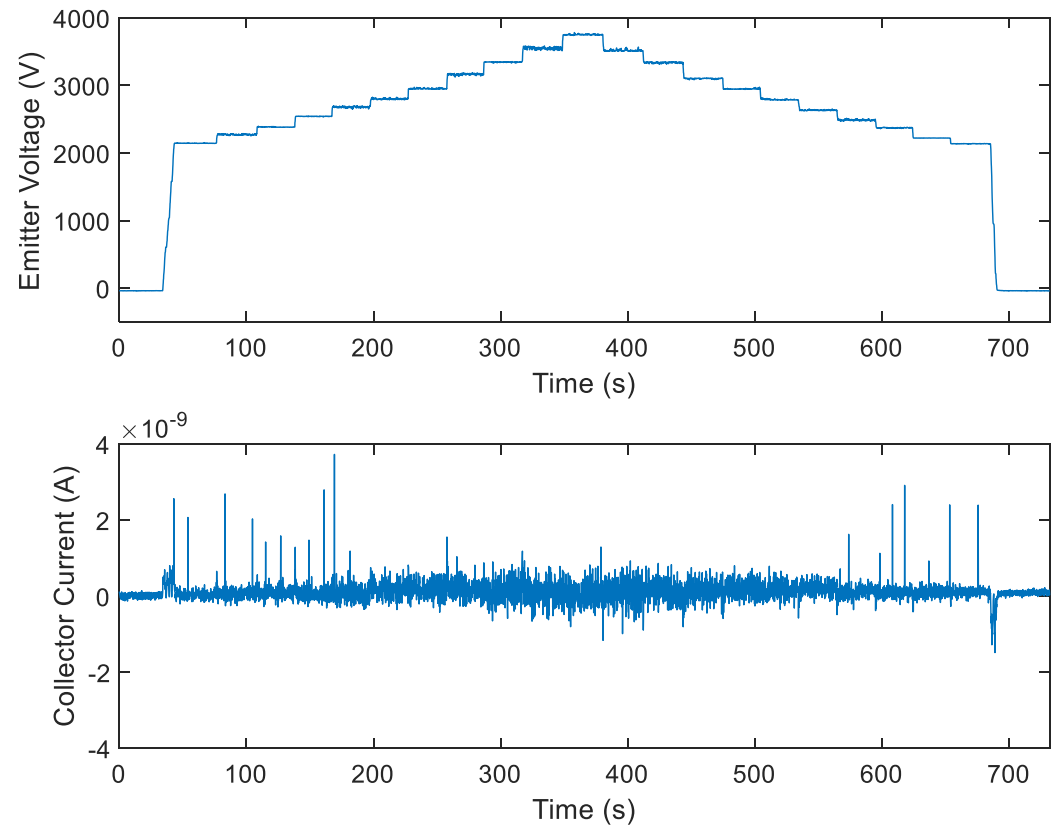

\section{Figure 3.18: Example Current Data from the Collector Plate of the Demonstration Thruster During a} Live Voltage Sweep.

Data collected from an example live sweep with the extractor current being measured is shown in figure 3.19. The current spikes seen here are significantly higher than those seen in the collector tests, with readings as high as $9.7 \times 10^{-8} \mathrm{~A}$ measured. The magnitude of the current spikes seem to be random across the voltage range for the most part, as there is no clear trend relative to voltages. The currents measured are certainly affected by a similar charge loss situation as with the collector run in some capacity. Something worth noting about the timing of the spikes is that they are much more likely to occur when the voltage is raised to the next step, because the increased strength in the electric field will impart a greater force on the propellant on the tip of the emitter, which is formed as a bead and not always emitting. This pushes past a threshold that was previously unmet, causing emission though still in an unsteady mode. This occurred consistently through all the Demonstration thruster testing.

Throughout the five tests on each of the collection surfaces, the measured current response is consistent. The current magnitudes measured on the collector plate are within $5.1 \times 10^{-9} \mathrm{~A}$, while the current magnitudes measured on the extractor are within $2.0 \times 10^{-7} \mathrm{~A}$. There is a large variability of the magnitudes of these current peaks within these envelopes, due to the charge loss phenomenon. 

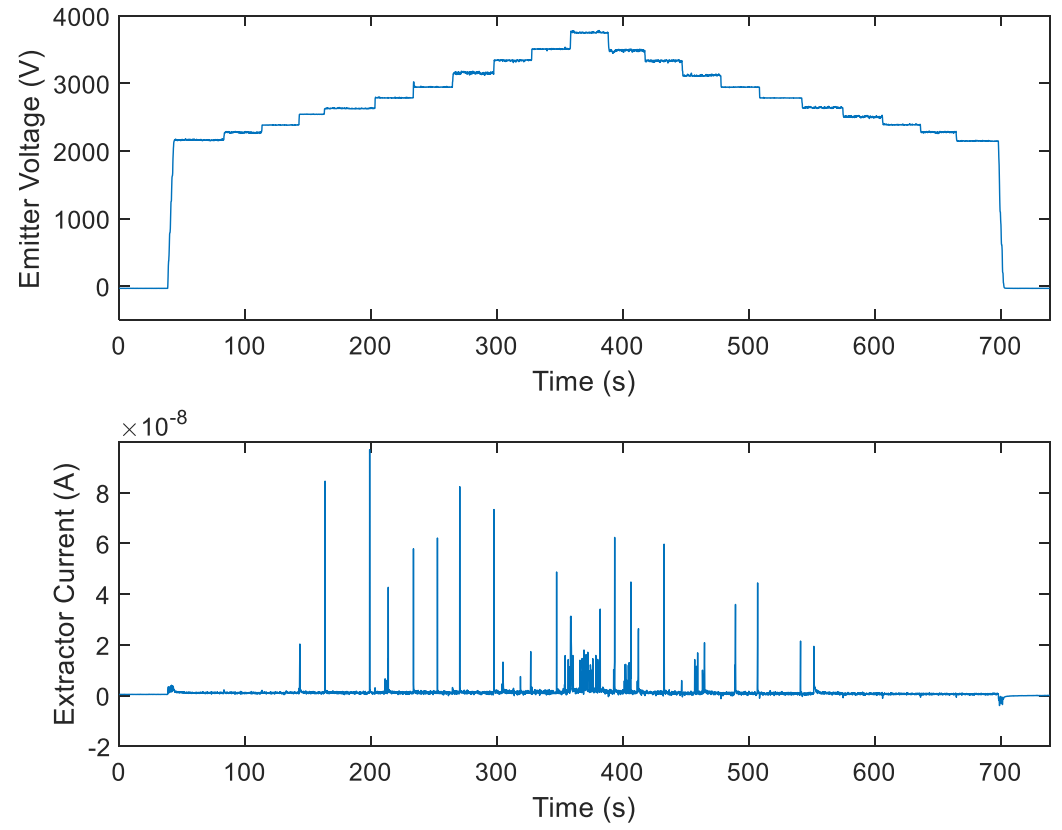

Figure 3.19: Example Current Data from the Extractor of the Demonstration Thruster During a Live Voltage Sweep.

An interesting difference between the tests collecting data on the collector plate and the tests collecting data on the extractor is that when the pico-ammeter was connected to the extractor, the propellant on the emitter was much less likely or unable to form a stable cone-jet at comparable voltages. A potential explanation to this is that the addition of the pico-ammeter in the conductive path from the extractor to ground introduces an interference that disrupts the flow of charge significantly enough to influence the formation of the expected electric field between the emitter and the extractor. There is no apparent reason that the ammeter should impede the circuit in this way, so this explanation is likely not sufficient in reality. This issue is manifested as the thruster appearing to operate a lower voltage compared to the test sweeps collecting data on the collector plate.

Another topic to be discussed is the sample rate of the testing diagnostics of $8 \mathrm{~Hz}$. A higher sample rate would have been preferred, however, in order to achieve background noise low enough to be able to measure amounts of current found in this system, the current sample rate was believed to be the best possible with the available setup. This results in data of the current spikes measured being composed of only a few data points at most. So rather than having points that make up the rise and fall of the emission, the data points may only catch one side of a current spike event. This is mostly apparent in the unsteady emission modes, 
where the current is emitted quickly in bursts and not steadily over time. For the testing set out for this thesis, this compromise was deemed acceptable, as confirmation of emission is achieved. The $8 \mathrm{~Hz}$ sample rate is approaching the hardware limit with digital communication with the pico-ammeter at the accuracies necessary for proper measurement. 


\section{Chapter 4}

\section{RESEARCH THRUSTER DESIGN PRINCIPLES}

Building on the successes of the Demonstration thruster, the Research thruster takes form as a thruster with much greater applicability to electrospray research at Cal Poly. The Research thruster incorporates features to enable many future research opportunities, while also having the simplicity, visibility, and durability that

will promote easy understanding and modification. In addition, certain design elements are influenced by the fact that the thruster is operated inside of a vacuum chamber. A prediction of basic performance is conducted to provide metrics to base testing procedures and better understanding how the thruster system operates.

\subsection{Design Choices}

The core concepts of modularity and visibility influence the Research thruster's design, in the effort to make the thruster as accessible as possible for the level of electrospray research to be conducted at Cal Poly. A clear side view of the array of emitters is permitted during operation. The testing of this thruster was conducted inside of a vacuum chamber to simulate the space environment and thus give a better understanding of the thruster's performance would it theoretically be operated in orbit. Though there is no intention of this design being used in a performance role, it is necessary in order to draw comparisons to designs in which it is the purpose as well as permit the diagnostics from working to begin with. A model of the Research thruster with labels of the major elements is given in figure 4.1 to reference throughout the discussion of the design choices made.

The primary feature of the Research thruster over the Demonstration thruster is the interchangeable emitter structure insert, referred to as the emitter block in this thesis. This component allows quick machining of different designs of emitter layouts and sizes, within a set dimensional limit. This limit was deemed acceptable for this phase in the electrospray research at Cal Poly as it allows for a significant amount of emitters to be aligned in a linear array, and just as easily accommodates research with a single emitter. Designs looking to make use of a large two dimensional array of would greatly benefit from a custom structure designed to that specific array, rather than a modular design. 


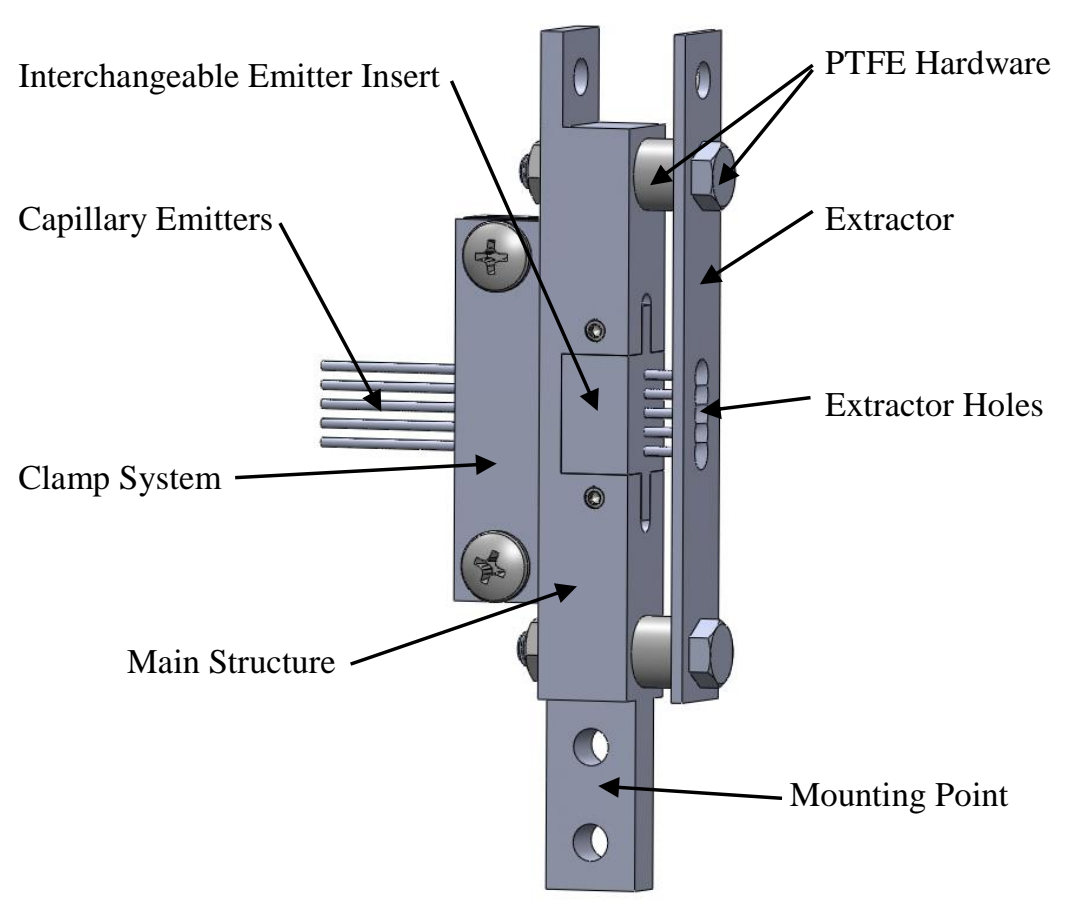

Figure 4.1: Assembled 3D Model of the Research Thruster, Showing Major Design Elements.

Internally wetted capillary needles were chosen as the emitters to direct propellant into the electric field. This is the simplest option for delivering propellant as it is largely independent of orientation and capillary size. No custom machining is necessary as these capillary tubes are readily available in a wide range of sizes. An externally wetted emitter would be unnecessarily difficult with the propellants expected to be utilized, and likely would not satisfy the goal of visibility well. There are similar concerns with using a porous emitter, but also poses issues with loading propellant, cleaning between testing, and the longevity of the emitters over repeated tests [6].

Utilizing the same design with different dimensions to accommodate the array of emitters, the Research thruster secures the emitters tightly in position through the use of aluminum clamps and silicone strips as gripping pads. Securing the emitters in this fashion allows for each individual emitter to adjusted manually, increasing the modularity of the system overall versus emitters being permanently mounted to the emitter block. Though this method can slightly increase the testing preparation time because each emitter does need to be aligned, the use of precision machined alignment spacers allows for all emitters to be aligned at the same time accurately. The friction of the clamps on the emitters is sufficient to prevent movement during proper handling. 
A syringe pump was utilized as the driver for an active propellant feed system that transports propellant stored in the syringe to the emitters inside the vacuum chamber. This is using the same hardware as used with the Demonstration thruster. Extensive experimentation was conducted attempting to have emitters be passively fed through a gravity driven design. An example of the thruster failures suffered during passive testing is shown in figure 4.2. Note that the downward direction in reality is to the right in figure 4.2. This was deemed unacceptable due to the inability to control flow rates of the propellant through the relatively large tubing, as well as complications with the propellant outgassing and creating disrupting air bubbles. An active system using a syringe pump can accurately control flow rates and can feature systems to reduce the propagation of air bubbles. For this experimentation, an offshoot from the main propellant line is used as a bleed line to remove bubbles before propellant is introduced to the thruster. It is worth noting that gas bubble formation in the propellant during testing of electrospray thrusters is very common across the entire field and often leads to failure, as explained by the presentation given by R. Wirz in May 2019 [29].

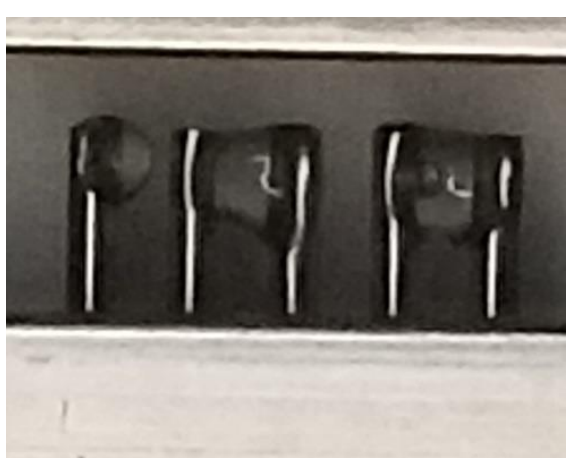

Figure 4.2: Example of Failure During Passive Propellant Feed Testing Due to Expanding Air Bubbles Causing Excessive Flow out of the Emitters.

The testing completed as part of this thesis utilized this thruster set up with a single emitter being operated, though the initial design of the emitter block features an array of five emitters as an expectation of a suitable path for future testing. On the extractor grid, the choice of the number of emitters requires an array of discreet holes aligned with the axes of the emitters. Some brief experimentation with all five emitters operating was attempted as well. Testing with simple and low-cost methods of achieving this goal were met with limited success in that only a few emitters would begin emitting before an event occurred that would necessitate the testing to be aborted. Figure 4.3 shows an example of a partial success of the array's operation. Note that the downward direction in reality is to the right in figure 4.3. An array of this size allows for the 
observation of emitters that surrounded by other operating emitters and those that are exposed to the edge of the array, similar to research conducted at Konkuk University, which looked at the interference caused by nearby emitters [26].

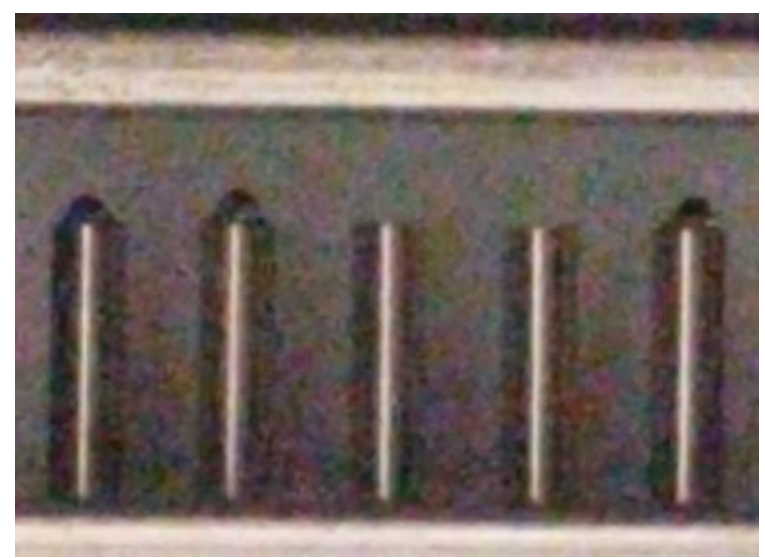

Figure 4.3: Attempt at Operating All Five Emitters of the Research Thruster at the Same Time Where only Three Emitters Became Active Before the Test Had to be Aborted Due to Arcing.

The majority of the testing completed with the Research thruster is done using un-doped glycerol as the propellant. This propellant has a long history of use with colloid thrusters, though it is usually doped with another chemical component to increase conductivity. Glycerol is a thick fluid with a high surface tension of $63.4 \mathrm{mN} \mathrm{m}^{-1}$ [30], which will require higher potentials to achieve emission relative to lower surface tension as is discussed in section 4.3. Glycerol also has a low vapor pressure, meaning it will not readily evaporate in a vacuum environment, which is a requirement for testing the thruster in the vacuum chamber. Additional testing as part of this thesis is completed using sodium iodide doped glycerol as propellant, which is a commonly used dopant [24]. This dopant will create a positively charged emission plume. Other dopants that could be used include, sulfuric acid, and creates a negatively charge emission plume. A negatively charged plume is not desirable for this testing as it would require a high voltage power supply capable of negative polarity or would require charging the extractor to high positive voltage, which would remove the capability to measure the interception currents. A negative polarity high voltage power supply was not available for this testing. For these reasons, the sodium iodide doped glycerol is utilized. An additive of $20 \%$ sodium iodide by weight to the glycerol is chosen as the mixture ratio which can increase the conductivity from $1 \times 10^{-6} \Omega^{-1}$ $\mathrm{cm}^{-1}$ un-doped [30] to $2.1 \times 10^{-4} \Omega^{-1} \mathrm{~cm}^{-1}$ doped [31]. Results for both propellant choices are compared both visually and quantitatively to get initial understanding of why propellant matter greatly for a colloid system. 


\subsection{Technical Specifications}

The Research thruster makes use of the much of the same stock material as the Demonstration thruster, such as the aluminum bar stock for the main structure, clamps, and extractor. This was made common between designs for reasons of simplicity and practicality: there was no apparent reason to change the design significantly after the successful testing of the Demonstration thruster. This design is able to be machined confidently within tolerances with standard tooling on a common mill. Despite the addition of the interchangeable emitter block, the Research thruster remains very durable and easy to assemble.

A three view diagram with major dimensions of the assembled Research thruster is given in figure 4.4. Many of these dimensions are retained from the dimensions of the stock material the components are machined from, such as the depth of 0.5 inches and the width of 1 inch for the main structure. A large width is necessary for the clamps to have enough contact surface area on both the emitters and the main structure. The width also allows for better alignment of the emitters, as will be discussed. A balance of durability, structural strength, and a slim profile is provided by the depth of 0.5 inches. The overall height is most determined by design needs; it had to be tall enough to fit an emitter block large enough to hold an acceptable rang of emitter array layouts and to machine this, room had to be given for the mill tooling for certain milling steps. A protruding tab of metal extends from the bottom of the thruster structure to allow for mounting on a support structure for use inside the vacuum chamber. Similarly, tabs extending above the main thruster structure and extractor were added to serve as mounting points for the wiring connections to allow for better electrical connection quality by using metal fasteners rather than the insulating fasteners.

Material choice is very important for proper vacuum chamber testing, where some materials can release latent gases they have absorbed through exposure to atmospheric pressures and water vapor [32]. This phenomenon is called outgassing and is a crucial consideration for all components that will be exposed to vacuum pressure environments. Aluminum and stainless steel are two very common metals used in vacuum applications because they outgas much less than other materials. All of the machined components of the Research thruster are fabricated from aluminum stock, and all metal fasteners and the emitters are made of stainless steel. For the necessary electrically isolating components between the extractor and the structure, PTFE fasteners were used for its favorable properties in vacuum. Silicone rubber was used for the clamp grip 
pads and the propellant tubing for its flexibility and its vacuum properties. Silicone is also used as the insulation for the wiring leading to the thruster components inside the vacuum chamber.

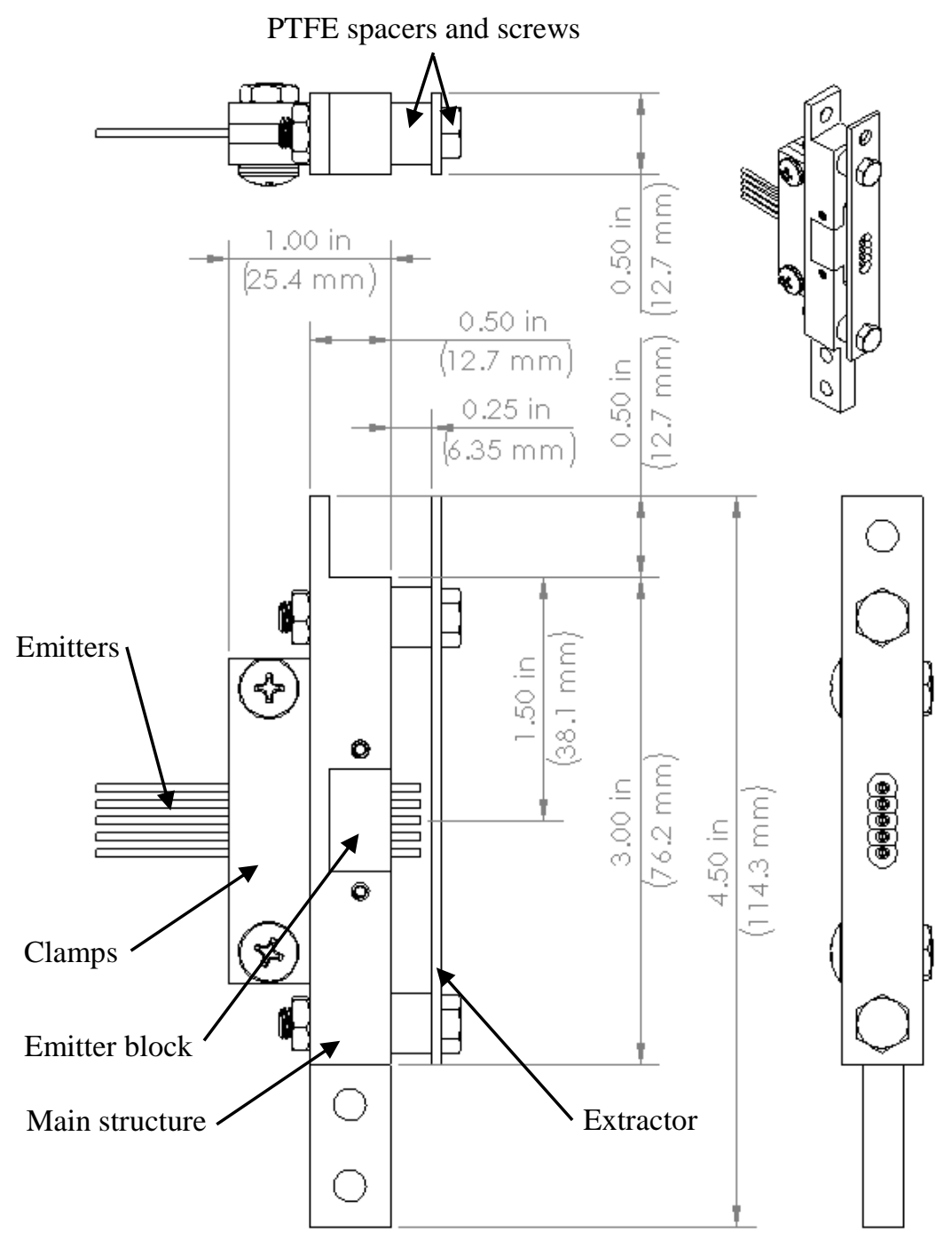

Figure 4.4: Three View Drawing with Major Dimensions and Isometric View of the Assembled Research Thruster.

The component that grants this design its modularity is the emitter block. It is designed to be easily machined and inserted into the main structure for testing with different emitters and arrays. This is accomplished by simple means of having a large milled out volume where the emitter block is inserted and secured by set screws. The side of the main structure where the emitters are clamped down has a channel milled out so the emitter block and emitters can go through either side completely. A close up view of the 
relevant component models is shown in figure 4.5. An area of $0.125 \times 0.625$ square inches is available for an emitter array design to utilize on this design.

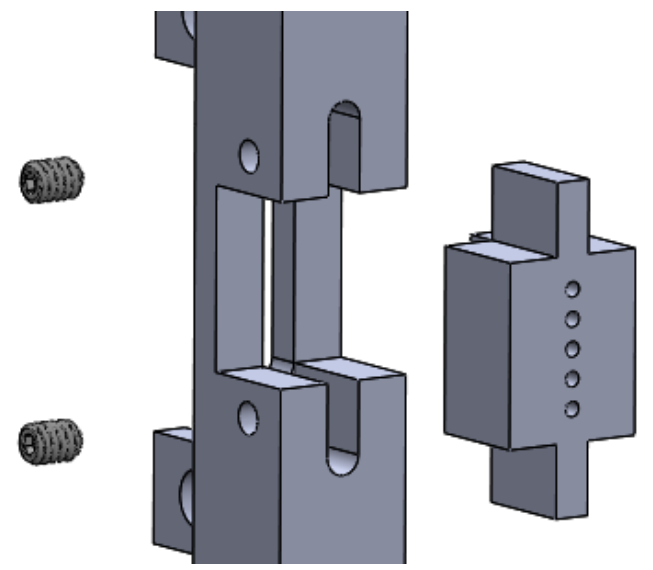

Figure 4.5: Close-Up View of Interchangeable Emitter Block Exploded Assembly on the Research Thruster.

The Research thruster uses the same diameter needle for the internally wetted emitters as with the Demonstration thruster: an 18 gauge stainless steel blunt tip dispensing needle. Stainless steel is a good choice for the vacuum chamber environment and is very chemically resistant. The only operation to make these needles ready as emitters is cutting them to length, much simpler than machining very small parts for an externally wetted or porous design. The inside diameter is 0.038 inches and the outer diameter is 0.050 inches. Visibility of the emission is even more troublesome with the Research thruster as it must be viewed through a viewport on the chamber. The size of these emitters will provide better visual confirmation of cone-jet formation as the thruster is operating. The emitter block retains the length of hole the emitters sit in, and so keep the same alignment accuracy as the Demonstration thruster design. An emitter block hole diameter of 0.052 inches and assuming a worst case tolerance addition of plus 0.002 inches results in a maximum angular misalignment of 0.458 degrees relative to the face of the main structure. Meaning that the maximum relative angular misalignment between emitters of 0.916 degrees, the same as with the Demonstration thruster.

The emitters are secured in their positions through the clamps and silicone grip pads which allows for easy customization of the distance between the tips of the emitters and the extraction grid. Alignment to a chosen separation is performed with precision machined spacers of the appropriate thickness. The chosen spacer was placed on the inner face of the extractor towards the main structure and the emitters are pressed 
into the spacer to be flush with it. Then the emitters are secured by the clamps and the spacer was removed. This method permits an accuracy of this separation of several thousandths of an inch.

As with the Demonstration thruster, the Research thruster extractor is designed in a way to minimize its apparent thickness from the perspective of the emission plume. Figure 4.6 shows a dimensioned cross section view of the emitter array and the extraction grid. A $0.5 \times 0.0625$ inches cross section aluminum bar was used to form the extractor to ensure minimal flexing would happen during assembly and alignment. This thickness also makes the component much more resistant to permanent damage by bending. An array of through holes was drilled into the extractor, aligning with the axes of the emitters. Each hole is countersunk to reduce the thickness of the extractor at the surface facing the emitter to 0.010 inches. This thickness would ideally be as thin as possible, but it was kept at 0.010 inches to maintain the strength of the component to avoid damage potentially caused through mishandling. At 0.076 inches in diameter, the extractor holes don't overlap, leaving an amount of material between the holes that also reduces the likelihood of damage in those regions of the extractor. The dimensions given in figure 4.6 show the emitter and extractor separation as the value used during testing, which is explained in the next section.

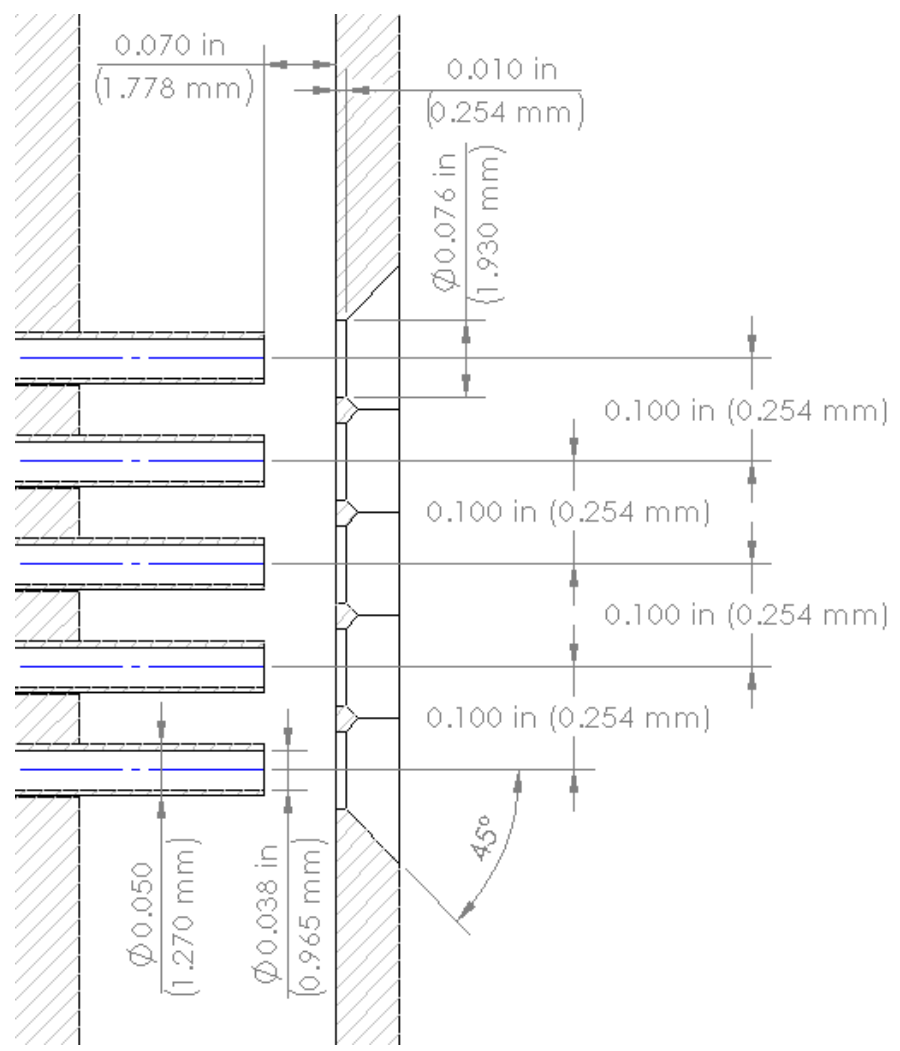

Figure 4.6: Close-Up, Cross-Section View of Emitter Array and Extractor Plate on the Research Thruster with Major Dimensions. 


\subsection{Thruster Performance Expectations}

Testing of the Research thruster for this thesis was planned to be primarily for the confirmation and discussion on the successful emission of the thruster. A simple prediction was made for the initial onset of emission using equations describing the physical phenomenon of the Taylor cone. An analysis of the voltage breakdown threshold was performed to ensure that arcing would not be an issue during nominal operation of the thruster in vacuum pressure conditions. The electric field lines are calculated using a simplified twodimensional analysis performed in computational fluid dynamics software. The information gained through these efforts is sufficient to define testing scenarios and explain the results to a reasonable level.

Calculation of the onset voltage for the Research thruster is performed in the same manner explained as for the Demonstration thruster. The difference is the specific details of the system: the dimensions and the propellant properties to be specific. Equation 2.8 establishes a lower bound for the onset voltage, and equation 2.9 establishes the upper bound. There is a significant disparity between the results of these equations due to the assumptions made in their respective derivations, though they attempt to define the same phenomenon. Because this prediction is a rough estimation to begin with, it would be unnecessary to treat these results as more than orders of magnitude calculations. Applying these equations with values describing the Research thruster system results in the plot shown in figure 4.7. The outer radius of the emitter $r_{t}$ is 0.025 inches $(0.635$

$\mathrm{mm}$ ) for the 18 gauge needle, which is used because it is assumed the propellant will wet the blunt face of the emitter as a worst case scenario. The surface tension $\gamma$ of glycerol, doped and un-doped, is $63.4 \mathrm{mN} \mathrm{m}^{-1}$ [30]. The distance between the emitters and the extraction grid $d$ is varied between in a range that is beyond the values reasonably expected to be used. 


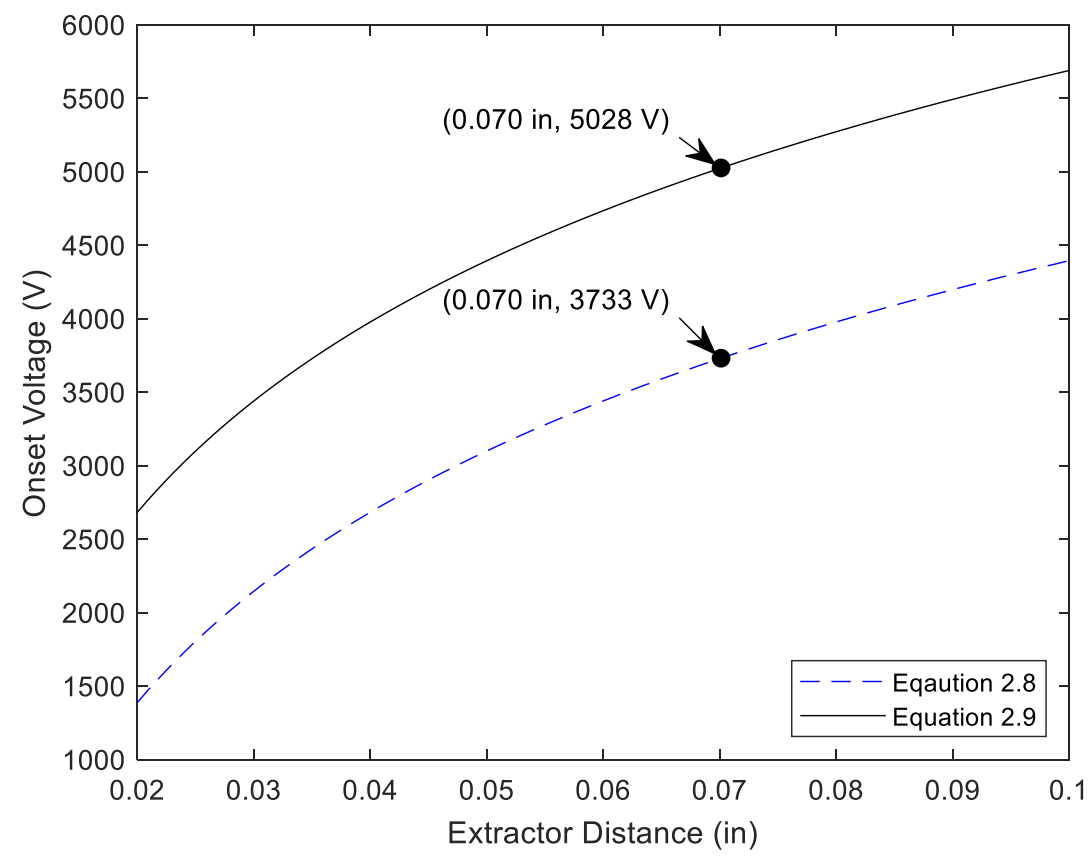

Figure 4.7: Onset Voltage Trend for the Research Thruster for a Range of Separation Distances Between the Emitters and Extractor.

Operation of high voltage in a vacuum environment can pose issues of arcing and even plasma formation in the operator is not careful about the placement of charged components and their proximity to conductive surfaces. To determine whether or not arcing would be an issue during normal operation of the Research thruster, an analysis of the theoretical breakdown known as a Paschen breakdown curve is performed for the expected vacuum conditions. The threshold for electrical discharge is given in equation 2.10. The pressure $p$ inside the vacuum chamber during testing is chosen to $2 \times 10^{-4}$ Torr as a worst case scenario for the specific chamber used in this testing. The electrode distance $d$ is varied to include values that would be present inside the vacuum chamber, such as the steel chamber walls. Constants $A$ and $B$ are found to be $11.25 \mathrm{~Pa}^{-1} \mathrm{~m}^{-1}$ and $273.75 \mathrm{~V} \mathrm{~Pa}^{-1} \mathrm{~m}^{-1}$, respectively, and the coefficient of secondary electron emission $\gamma_{s e}$ is 0.01 [21]. Looking at the resulting theoretical curve in figure 4.8, it is reasonable to assume there won't be any expected issues with arcing during normal operation of this thruster.

Comparing the theoretical breakdown threshold with the onset voltage calculations shows that the decision of which distance separating the emitters and the extractor boils down to making it as small as possible without risking arcing by the propellant being pulled onto the extractor by the force of the electric field. An understanding of the protrusion of the propellant was gained from the Demonstration thruster tests 
established a loose lower bound of around 0.05 inches $(1.27 \mathrm{~mm})$. The desire to keep the voltage as low as possible to accommodate the aging power supply established a loose upper bound of around 0.09 inches $(2.286 \mathrm{~mm})$. Within these bounds a nice middle ground of 0.070 inches $(1.778 \mathrm{~mm})$ was chosen to be the distance between the emitters and extractor during testing.

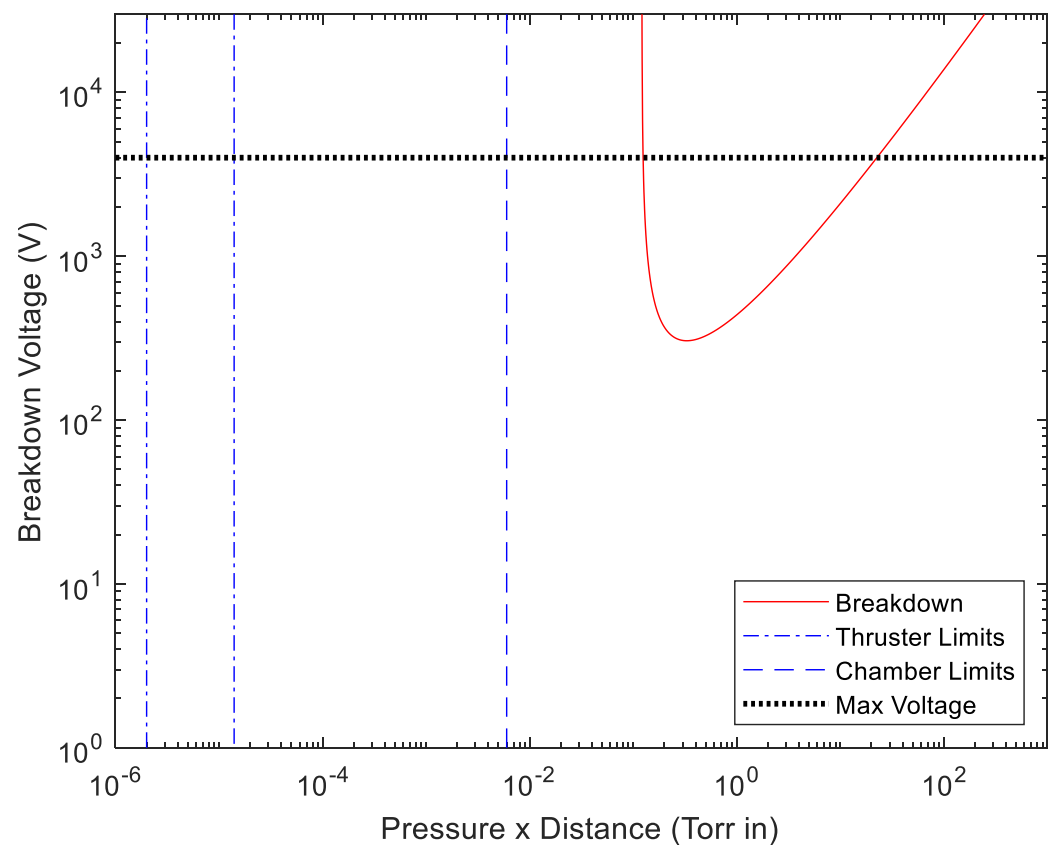

Figure 4.8: Calculated Paschen Breakdown Curve in Air with Relevant Values for the Research Thruster.

The formation of the electric field lines for the Research thruster are computed using the CFD-ACE computational fluid dynamics program, just as is done with the Demonstration thruster. A simplified twodimensional representation of the thruster meshed into the program with the conditions of a constant high voltage applied to the emitters and a grounded extractor. Figure 4.9 shows the resulting map of the electric field vectors. The figure focus on the center emitter amongst the array of five, as this is the emitter used during testing. Additional details of the CFD analysis for the Research thruster can be found in appendix A. For the scenario depicted, the distance between the emitters and the extractor is set at 0.070 inches $(1.778$ $\mathrm{mm}$ ), and the emitters are charged to positive $3700 \mathrm{~V}$ while the extractor is set to $0 \mathrm{~V}$. The emitter tips and propellant are assumed to be blunt to make visualization easier and the meshed model simpler, though this is not accurate as the propellant will form a conical shape as it's pulled on by the electric field. The same curling of the electric field lines as in the Demonstration thruster analysis is seen, as would be expected since the 
extractor designs are very similar. This means that low energy particles from the emission plume are likely to follow those lines are impact the extractor, leading to performance losses and potentially failure if the propellant builds up on the extractor enough to cause a blockage or arc to the main body of propellant.

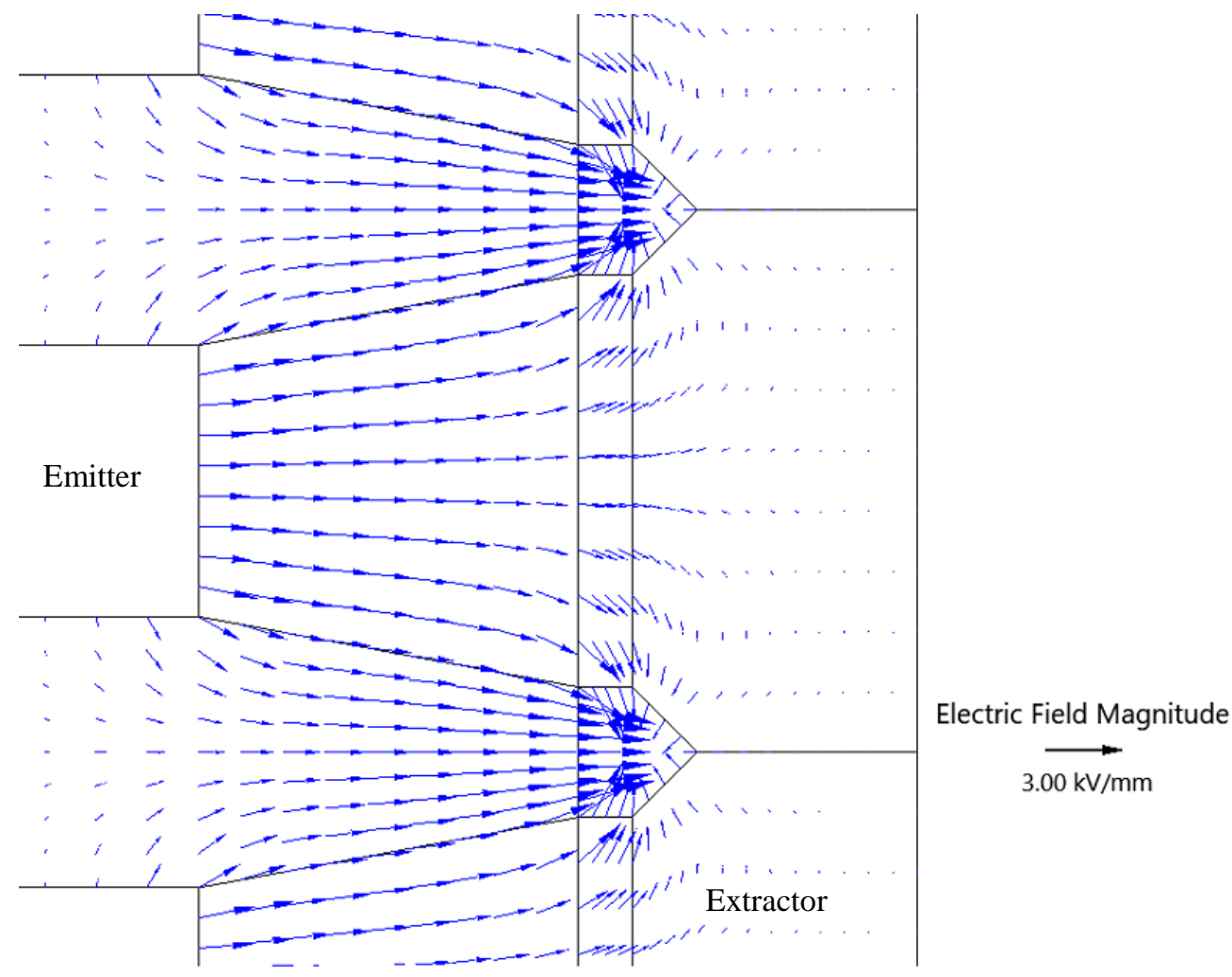

Figure 4.9: Vector Plot of the Electric Field Strength near the Center Emitter and Extractor for the Research Thruster. 


\section{Chapter 5}

\section{RESEARCH THRUSTER TESTING, RESULTS, AND DISCUSSION}

The testing of the Research thruster as part of this thesis is the beginning of the efforts that can be accomplished the current setup. The desired outcomes are verification that the thruster is emitting consistently and in a manner worth further work. This is accomplished visual means of observing the emissions, and by collecting current data from the thruster's extractor and a collection plate that captures the emission plume. This gives a comparison to how much propellant is being intercepted by the extractor and thus not contributing to thrust. The majority of testing completed is utilizing un-doped glycerol as propellant, and additional brief testing is completed using glycerol doped with sodium iodide.

\subsection{Experimental Setup and Diagnostics}

A basic diagram representing the electrical and propellant connections is shown in figure 5.1. Direct current measurements are taken from either the collector plate or the extractor, with the emitters being charge to high positive voltage to create the electric field necessary for emission.

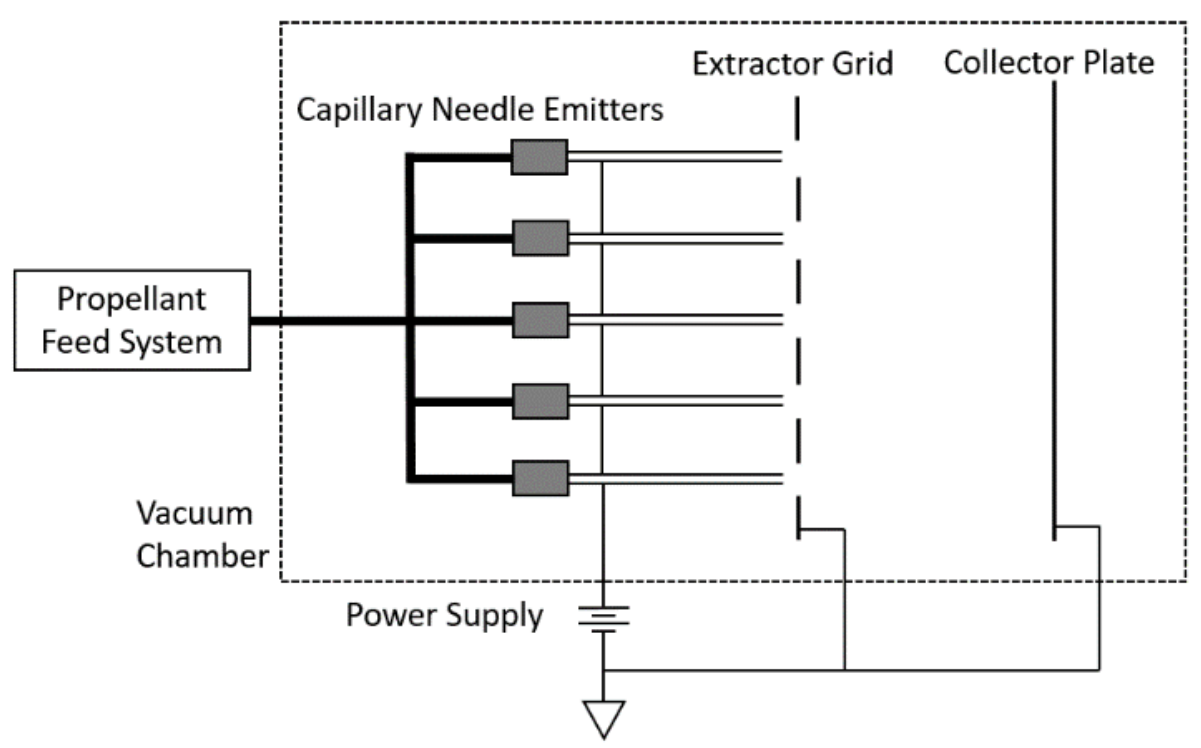

Figure 5.1: Simplified Electrical Diagram Connecting the Components of the Research Thruster. 
All equipment used in the testing setup for the Research thruster is shared from the equipment used in testing the Demonstration thruster. A Glassman High Voltage Inc. EL Series power supply, Model EL10P04.0, was used to charge the emitters to the necessary voltages for emission. The power supply is capable of outputting voltages as high as $10 \mathrm{kV}$ at up to $4 \mathrm{~mA}$, more than sufficient for the testing of the Research thruster. To measure currents, a Keithley Model 6487 pico-ammeter was employed to deliver femto-Amp digit measurements. Since the Glassman power supply is analog only, a Keysight Infiniivison Oscilloscope, Model DSOX2004A with a 1000:1 probe was used to take measurements of the output voltage for data recording. The propellant feed system uses the New Era Pump Systems NE-300 Just Infusion Syringe Pump as the driving force for delivering propellant to the emitters. All of this equipment is situated around the vacuum chamber used to create the vacuum environment, shown in figure 5.2. Known as "Big Green," this chamber is capable of producing vacuum pressures as low as $7 \times 10^{-5}$ Torr in ideal conditions through a combination of a roughing pump and a turbo pump. With the Research testing apparatus inside of the chamber and propellant loaded, the chamber would reach an average vacuum of about $1.3 \times 10^{-4} \mathrm{Torr}$. Images and video were captured using the camera on a Samsung Galaxy S8 smartphone.

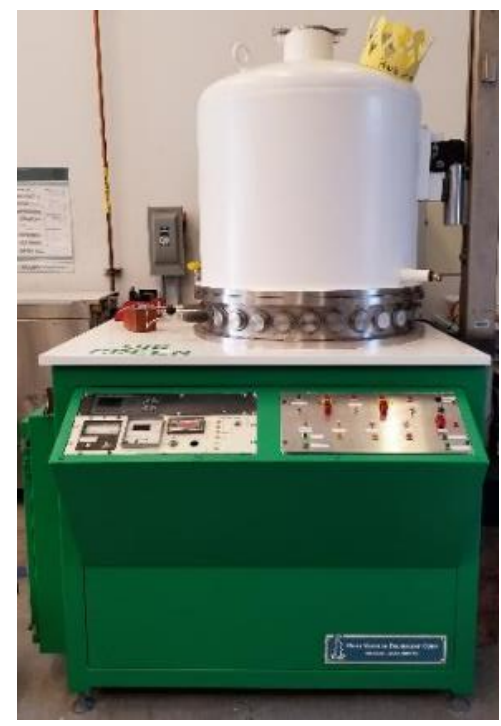

Figure 5.2: Vacuum Chamber Used for the Testing of the Research Thruster, Known as "Big Green."

All the electrical and fluid connections connect to the thruster through the vacuum chamber walls using several feedthroughs. Figure 5.3 shows a diagram with the semi-accurate relative positions of the equipment around the vacuum chamber and the electrical and fluid paths in greater detail. The high voltage connection is made through a series of RG-8U cabling and 22 AWG high voltage wire on the outside of the 
chamber. The high voltage passes through the chamber wall using a custom-fabricated electrical feed-through fashioned from a 1 inch stainless steel baseplate bolt and features a 0.0625 inch $(1.586 \mathrm{~mm})$ diameter copper rod as the conductive path. This HV feed-through is insulated with a minimum thickness of 0.1563 inches (3.97 mm) of either PEEK (polyetheretherketone) or PTFE. Connections to the HV feed-through are made using copper alligator clips on both inside and outside of the chamber. Current measurement paths leading from the pico-ammeter to both the extractor and the collector plate are connected using standard BNC cables on the outside of the chamber. These connections are fed into the chamber through two $\mathrm{N}$-type feedthroughs with BNC adaptors attached on both the inside and outside of the chamber. Electrical connections on the inside of the chamber are all done using the $22 \mathrm{AWG}$ HV wiring, insulated to $40 \mathrm{kV}$ with silicone. These are connected to the thruster components using stainless steel hardware and tin-coated copper ring terminals.

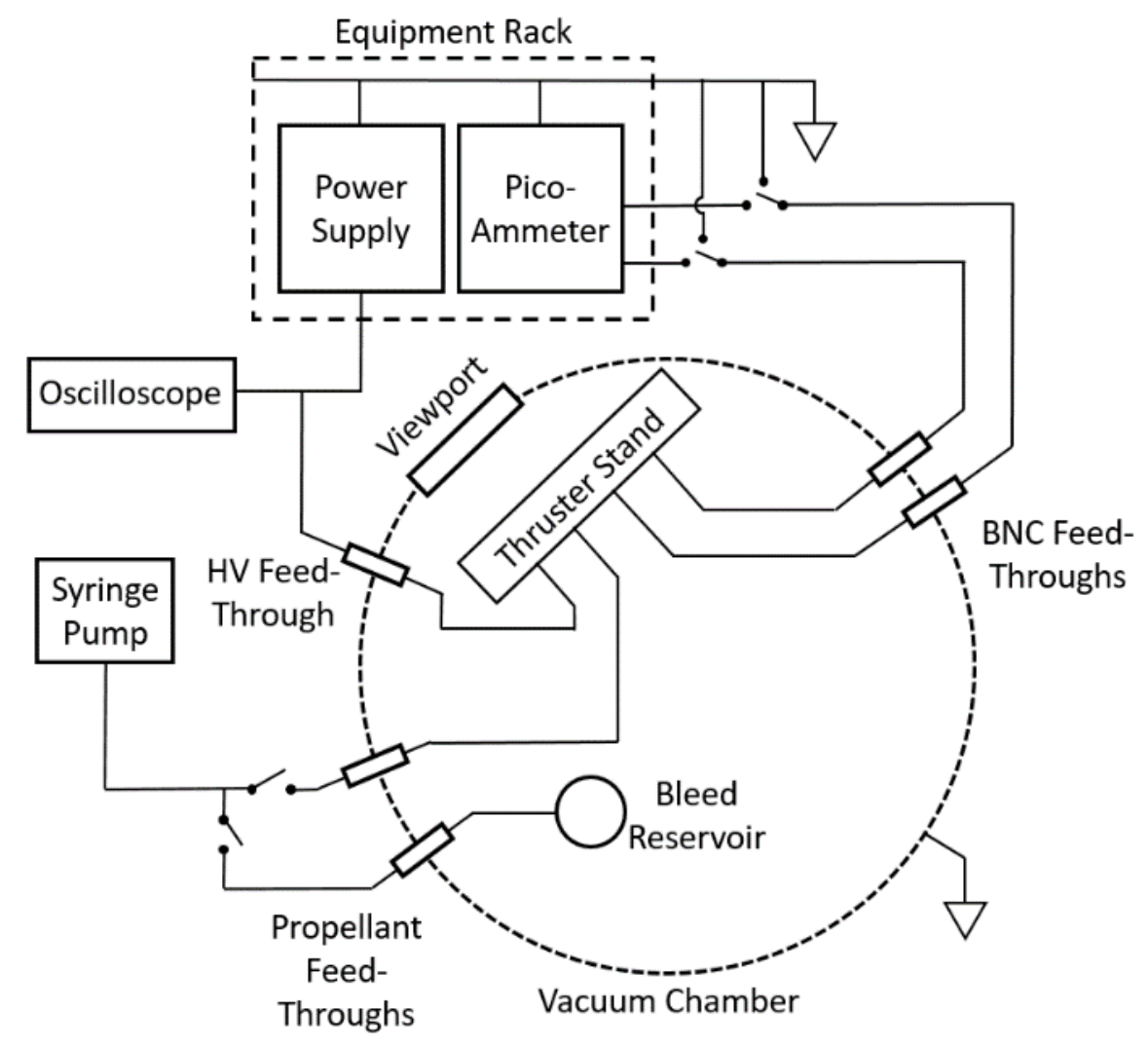

Figure 5.3: Block Diagram of Electrical and Propellant Paths and Equipment Used for the Research Thruster Testing.

Propellant transport both inside and outside of the chamber is accomplished using silicone tubing with an inner diameter of 0.039 inches $(1 \mathrm{~mm})$ and an outer diameter of 0.118 inches $(3 \mathrm{~mm})$. A picture of the external propellant feed system is shown in figure 5.4. A gas tight glass syringe of $10 \mathrm{~mL}$ internal volume 
is used as the propellant reservoir and is secured tightly in the syringe pump to prevent the plunger and barrel of the syringe from moving relative to each other due to the suction created by the vacuum. The propellant is fed through a custom built system that direct flow to either the thruster or a bleed line that expels propellant to remove air bubbles from the main propellant line. Flow is split using a nickel-coated brass tee and stopped entirely by engaging a metal clamp that seals the silicone tubing in on itself, creating an air-tight barrier. The two propellant paths are fed into the chamber using two custom feedthroughs fashioned from Swagelok 0.5 inch stainless steel tubing caps, featuring 0.625 nominal tubing for the propellant to move through. These fluid feedthroughs are also insulated with at least 0.1563 inches $(3.97 \mathrm{~mm})$ of either PEEK or PTFE because of the conductive nature of the propellant; the charge from the power supply is distributed throughout the entire body of propellant. An image of the custom fluid feedthroughs is in figure 5.5. The silicone tubing provided a great seal on the tubing components and did not affect the base pressure of the chamber. On the inside of the chamber, the main propellant line leads to the emitter needles and the bleed line is contained in a glass cylinder.

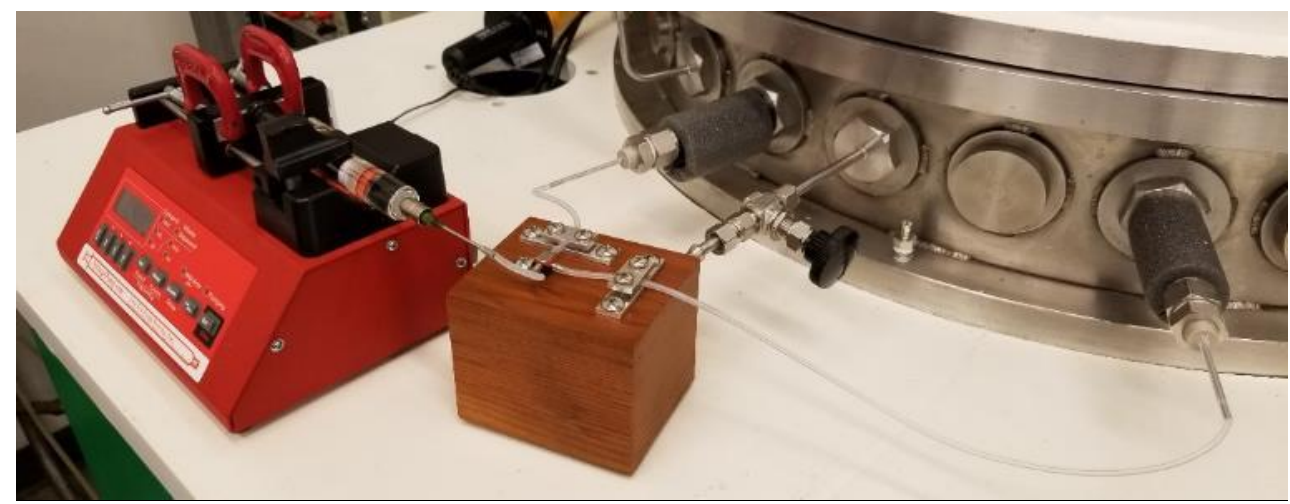

Figure 5.4: Syringe Pump and Propellant Bleed System Connected to Fluid Feedthroughs on the Vacuum Chamber.

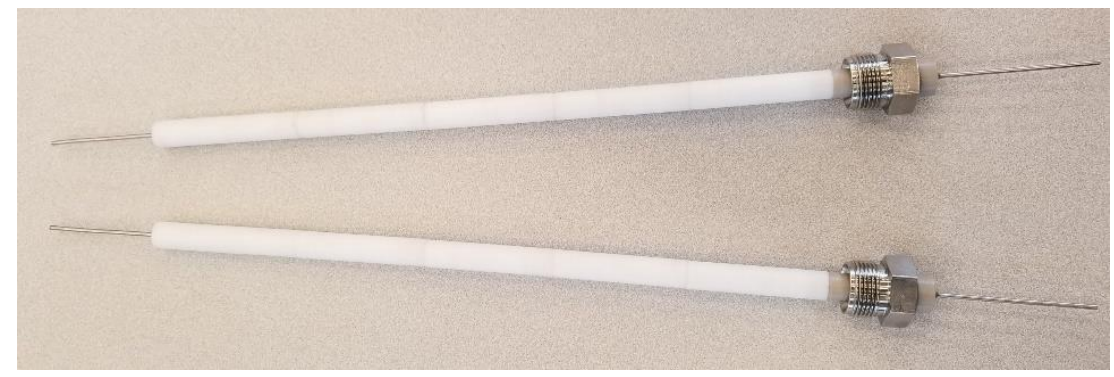

Figure 5.5: Custom Fluid Feedthroughs Making Use of 1/2 Inch SwageLok Tubing Caps. 
For the testing conducted in this thesis, flow resistance was added to the main propellant line in the form of a porous wad. This addition was shown to improve testing quality and reduce the chance of failure somewhat by preventing air bubbles from travelling to the emitter tip. The drawback is an impaired ability to accurately control the flow rate of propellant to the emitter tip, as the resistance will cause pressure to build up behind it, potentially changing the observed flow rates relative to the setting on the syringe pump. For the depth of testing to be conducted for this thesis, this was determined acceptable in order to have increased chance of stable emissions from the thruster by reducing the interference of air bubbles.

The Research thruster assembly complete with support structure and situated in the vacuum chamber is shown in figure 5.6. It was necessary to raise the emitters to the level of the viewport on the chamber in order to get good pictures and video of the thruster operating. The main thruster structure is mounted to the support structure with PFTE plates to isolate the voltage between the two metal surfaces. This structure also serves to mount the emission containment unit, which prevents unnecessary deposition of propellant onto the rest of the chamber and contained the collection plate. The collection plate is isolated from the box using silicon strips and PTFE machine screws. A strip of PTFE plate was attached with Kapton tape behind the emitters to create an opaque break drop for recording video of the thrusters operation. An aluminum drip pan was situated underneath the thruster to catch any large droplets falling from the thruster. Kapton tape was also used to create a channeling funnel that would prevent any droplets of propellant from contacting the PFTE spacer below the emitters by redirecting it to fall into the drip pan below. 


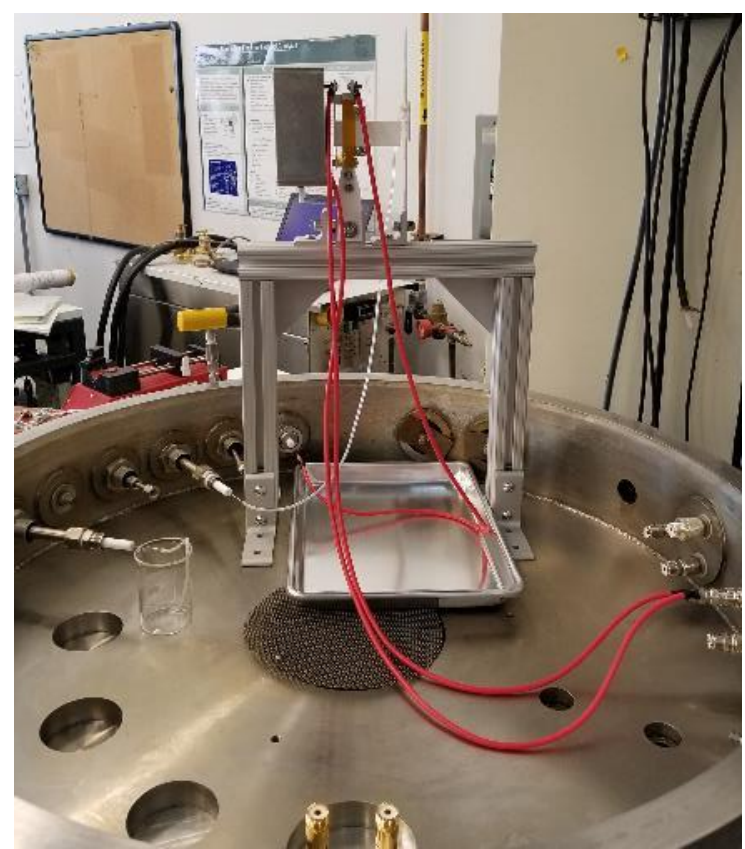

(a)

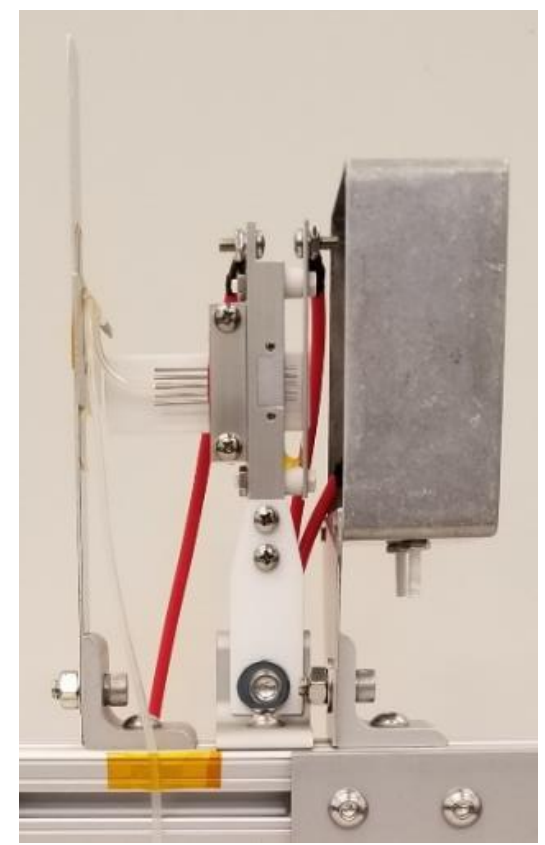

(b)

Figure 5.6: Assembled Complete Testing Apparatus Set up in Vacuum Chamber (a), and Close-Up of the Research Thruster on the Support Structure (b).

\subsection{Testing and Results}

Information from the calculations and analysis done influences the physical testing of the Research thruster system in the form of determining a suitable test procedure. Execution of this procedure results in visual and numerical data that can be analyzed and discussed in decent depth, though the primary goal is confirmation of successful emission. Confirmation is achieved visually and through current measurements from the picoammeter.

\subsubsection{Basic Testing Procedure}

A basic preparation and operation procedure is given here, a more detailed description is given in appendix B. With the thruster properly situated inside the vacuum chamber and all the necessary connections are made, the testing procedure can begin. The diagnostics equipment is given at least 30 minutes to warm up after being powered on, as is recommended by the appropriate user's manuals. This warmup period can coincide 
with the pumping procedure of the vacuum chamber. During this pump-down period, the propellant bleed line is open and the main propellant line is closed to allow for expanding air bubbles to be released without going through the emitter and potentially depositing propellant on the extractor or any other surface that may lead to failure once testing is begun. Once the chamber is near its base pressure, the dry voltage sweep tests for each collection surface are conducted. Before each live test, at least one dry test for each the collection plate and the extractor was performed as confirmation that the data collection circuit is working properly and there is not abnormal interference. Additionally, the dry tests would reveal if the power supply was outputting the expected voltages relative to the analog input on the dial, as this output would often change over time. If changes to the voltage sweep values were necessary because of this inconsistency, they could made here.

Dry testing for the Research thruster consisted of a voltage sweep from about $2600 \mathrm{~V}$ to $4500 \mathrm{~V}$ in 13 steps with 10 seconds spent at each step for both the rise and fall of the sweep. This range of voltages was chosen to be well beyond the expected voltages seen during the live testing. An understanding of the background noise present in the system due to the high voltage is gained this way. The voltage sweep for live testing ranges from $4000 \mathrm{~V}$ to about $4600 \mathrm{~V}$ in 4 steps with at least 30 seconds spent at each voltage step. The starting voltage of $4000 \mathrm{~V}$ was chosen so that there would be little doubt the propellant would emit. The upper limit was deemed acceptable and chosen to ensure a full sweep could be performed before any unexpected issue required the test be aborted. Current data was collected at $0 \mathrm{~V}$ before and after the voltage sweep for at least 30 seconds to obtain an offset value of the pico-ammeter to use if necessary.

To begin the live testing, the syringe pump is turned on to a high rate of $80 \mu \mathrm{L} / \mathrm{min}$ to clear the lines of air bubbles through the bleed line. Once the propellant is flowing free of bubbles, the bleed line is closed and the main propellant line is opened. A short waiting period is endured while the propellant reaches the thruster components. When the propellant is at the wad providing flow resistance, the pumping rate is reduced to its testing speed of $10 \mu \mathrm{L} / \mathrm{min}$ and more waiting time is necessary to reach the emitter. At a time about 2 minutes before the propellant is expected to reach the tip of the emitter, the LabView data collection software is initiated and the voltage is raised to the first step of the voltage sweep of $4000 \mathrm{~V}$. While sitting at this voltage, the propellant will eventually reach the tip and begin emitting and the voltage sweep can be continued as normal. After the sweep is completed, the thruster was kept running at $4000 \mathrm{~V}$ until signs of a failure were observed, at which point the voltage was brought to ground and testing concluded. 
Due to the setup only capturing the current on one surface at a time, and only one sweep able to be performed for each chamber pump down, several repeated tests were completed to gather sufficient data on both the extractor and collector surfaces as well as with the doped propellant. Between each of these tests the thruster must be thoroughly cleaned and the propellant lines purged to prevent premature failures resulting from deposited propellant or large air bubbles forming in the tubing. In all, four tests with un-doped glycerol were completed (two while collecting data from the collector plate and two while collecting data from the extractor) and one sweep with the doped glycerol (while collecting data from the collector plate) to serve as a brief comparison.

The primary difference between the testing procedures for un-doped glycerol and that of the glycerol doped with sodium iodide is that the propellant must be mixed before loading into the syringe. Sodium iodide is toxic to the environment and so any waste must be collected as hazardous waste. The weight of glycerol is measured and a corresponding $20 \%$ of that weight in solid sodium iodide is added to the glycerol. Complete mixing can take over 20 minutes and creates a significant amount of small air bubbles that are trapped in the thick propellant. These bubbles don't pose much of a threat as they tend to gather near the highest point in the syringe barrel overtime and so do not travel through to the propellant lines. A standard operating procedure for the handling and mixing procedure of the sodium iodide chemical is included in appendix $\mathrm{C}$.

\subsubsection{Visual Thruster Operation}

All live testing runs were recorded on video from the first emission to the conclusion of abortion of the testing. Note that the emitter orientation shown in the figures is not the orientation the thruster was operated in and is purely for formatting reasons. The emitters were operated horizontally. In the picture's orientation, the direction to the right of the emitter is downward in reality.

The un-doped glycerol testing for the Research thruster showed the propellant almost always emitting in an unsteady cone-jet at all voltages tested, for both setups where the pico-ammeter was connected to the collector plate or the extractor. An example of this emission is given in figure 5.7 over a time period of about three seconds. The rate of oscillation between emission and not is much faster than was experienced with the Demonstration thruster. Perhaps a combination of the glycerol's viscosity and surface tension cause 
it to release less propellant during each emission event, and so the amount removed at the tip of the emitter is quickly replaced by the flowing propellant, thus causing the higher frequency of emission during the unsteady formation. During emission the cone appears to be very sharp, hinting at a Taylor cone forming during these emissions, which is better to have than a purely dripping mode of emission. The peak of the cone also looks well aligned with the axis of the emitter needle, meaning the alignment with the extractor is satisfactory and thus the intercepted current would be expected to be low. It is likely that a steady cone-jet could be achieved at these voltages if the flow rate had been varied as well.

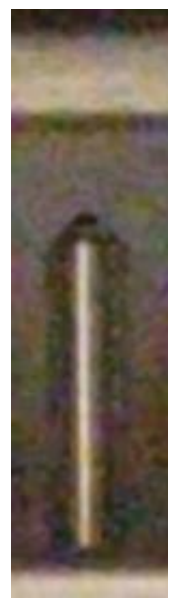

(a)

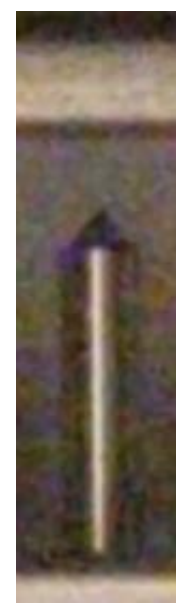

(b)

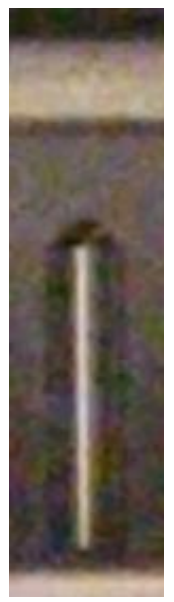

(c)

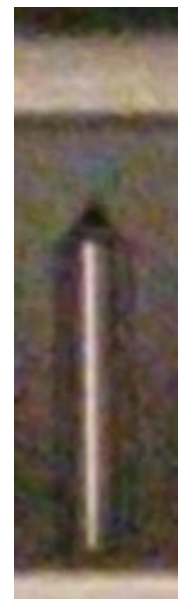

(d)

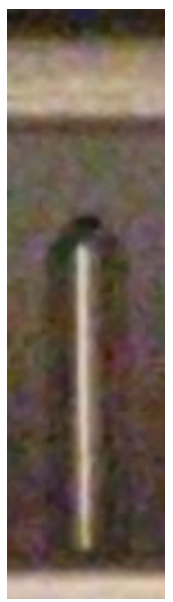

(e)

Figure 5.7: Progression Over 3 Seconds of the Research Thruster Emitting Un-Doped Glycerol in the Unsteady Cone-Jet Mode with the Emitter Charged to $4000 \mathrm{~V}$ : Approximately at Relative Times 0 s (a), $1 \mathrm{~s} \mathrm{(b),} 1.5 \mathrm{~s}(\mathrm{c}), 2 \mathrm{~s}(\mathrm{~d})$, and $3 \mathrm{~s}(\mathrm{e})$.

As the voltage was increased during the test sweep, the shaped and length of the cone-jet did not appear to change much. This progression from $4000 \mathrm{~V}$ to $4600 \mathrm{~V}$ is shown in figure 5.8. The pictures in this figure are taken during the moments of emission within the unsteady cone-jet. It is likely that the range in voltage was too narrow to reveal a noticeable change in a relatively low conductivity propellant such as glycerol. The cone remains on axis and about the same height throughout, and the frequency of emission did not noticeably change, though this was not analyzed in great depth. The addition of a high-speed camera may reveal slight differences between the thruster operation at each of these voltages, but that would reveal much more about all situations captured during this testing. 


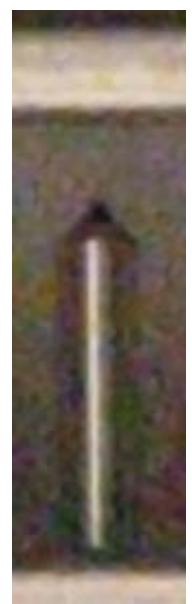

(a)

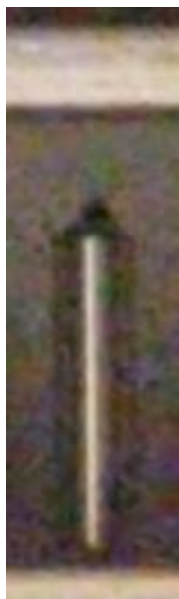

(b)

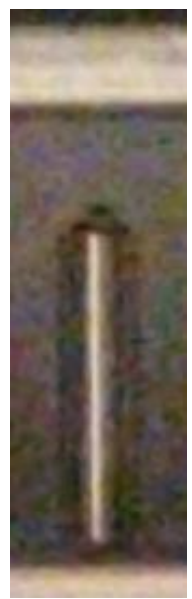

(c)

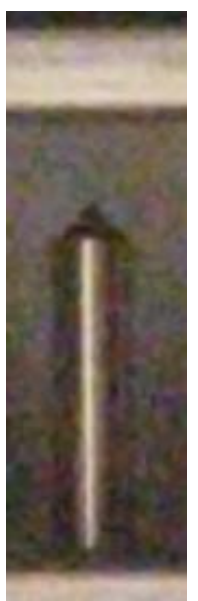

(d)

Figure 5.8: Unsteady Cone-Jet Formation of the Research Thruster Using Un-Doped Glycerol with the Emitters Charged to Different Voltages: at $4000 \mathrm{~V}$ (a), $4200 \mathrm{~V}$ (b), $4400 \mathrm{~V}$ (c), and $4600 \mathrm{~V}$ (d).

There did occur a time period during testing with the un-doped glycerol where a steady cone-jet was formed. This happened several minutes after the voltage sweep had been completed and the syringe pump had been turned off. This cone-jet formation at $4000 \mathrm{~V}$ is shown over a four second period in figure 5.9. With the pump off, the flow rate would have only been decreasing, meaning the reduced flow rate may have been what allowed the stable cone-jet to form. However, about 20 seconds after the cone-jet had become steady an air bubble disrupted the flow of propellant through the emitter. This bubble was likely present during most of the emission during this testing, and when it reaches near the tip of the emitter, it will begin to expand slightly due to the difference in pressure between the bubble and the vacuum chamber. This expansion would server to potentially increase the flow rates past the nominal value, and so perhaps an increased flow rate was necessary to achieve steady emission. This only occurred once and so the causes can't be determined from the single observation.

Unlike the Demonstration thruster testing, there was no visually observable difference in the emission between tests collecting data from the collection plate and those from the extractor. Figure 5.10 shows the two testing scenarios side by side at similar voltages; the collector plate example had the emitters set at $4600 \mathrm{~V}$ and the extractor example had the emitters set at $4560 \mathrm{~V}$. The cones during emission within the unsteady cone-jet look almost identical. This could be explained by the vacuum environment reducing the impact of having the pico-ammeter in the circuit on the emission quality. 


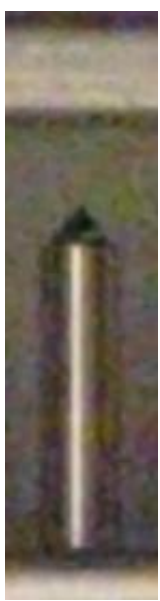

(a)

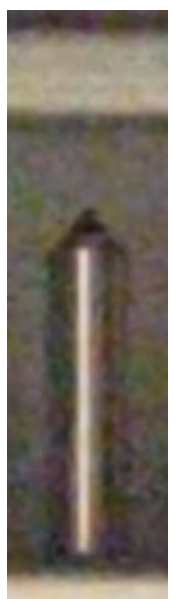

(b)

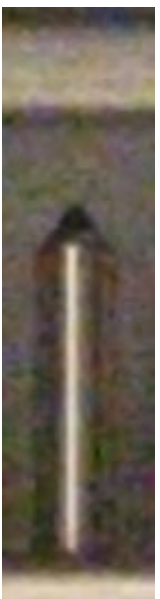

(c)

Figure 5.9: Progression Over 4 Seconds of the Research Thruster Emitting Un-Doped Glycerol in the Steady Cone-Jet Mode with the Emitter Charged to 4000 V: Approximately at Relative Times 0 s (a), $2 \mathrm{~s}(\mathrm{~b})$, and $4 \mathrm{~s}$ (c).

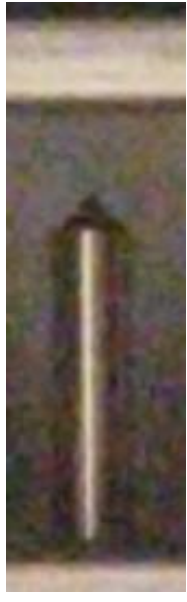

(a)

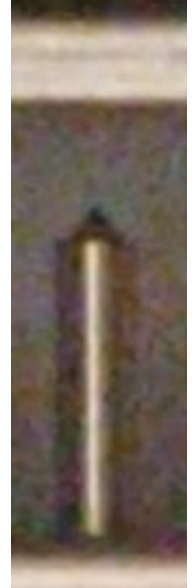

(b)

Figure 5.10: Comparison of the Research Thruster's Emission Quality Between Tests Using Un-Doped Glycerol Where Current is Collected from the Collector Plate with the Emitters at $4600 \mathrm{~V}$ (a) Versus from the Extractor with the Emitters at $4560 \mathrm{~V}$ (b).

Examples of the collector plate after testing are shown in figure 5.11. Enough propellant was deposited in a tight area to cause it to drip down the surface, and even off the collector entirely. A crude estimation of the beam divergence angle could be attained by relating the size of the deposition circle to the geometry of the emitter's position relative to the collector plate. However, because some deposited propellant would be undetectable to the naked eye, it was not worth pursuing within the scope of this thesis. It's interesting to note the differences between tests of similar lengths of time. There is a significant amount of conductive material with view factors of the emissions as it travels from the emitter to the collection plate, 
and so it is likely that even minute changes in the charge of these surfaces will affect the trajectory of the charged propellant droplets.
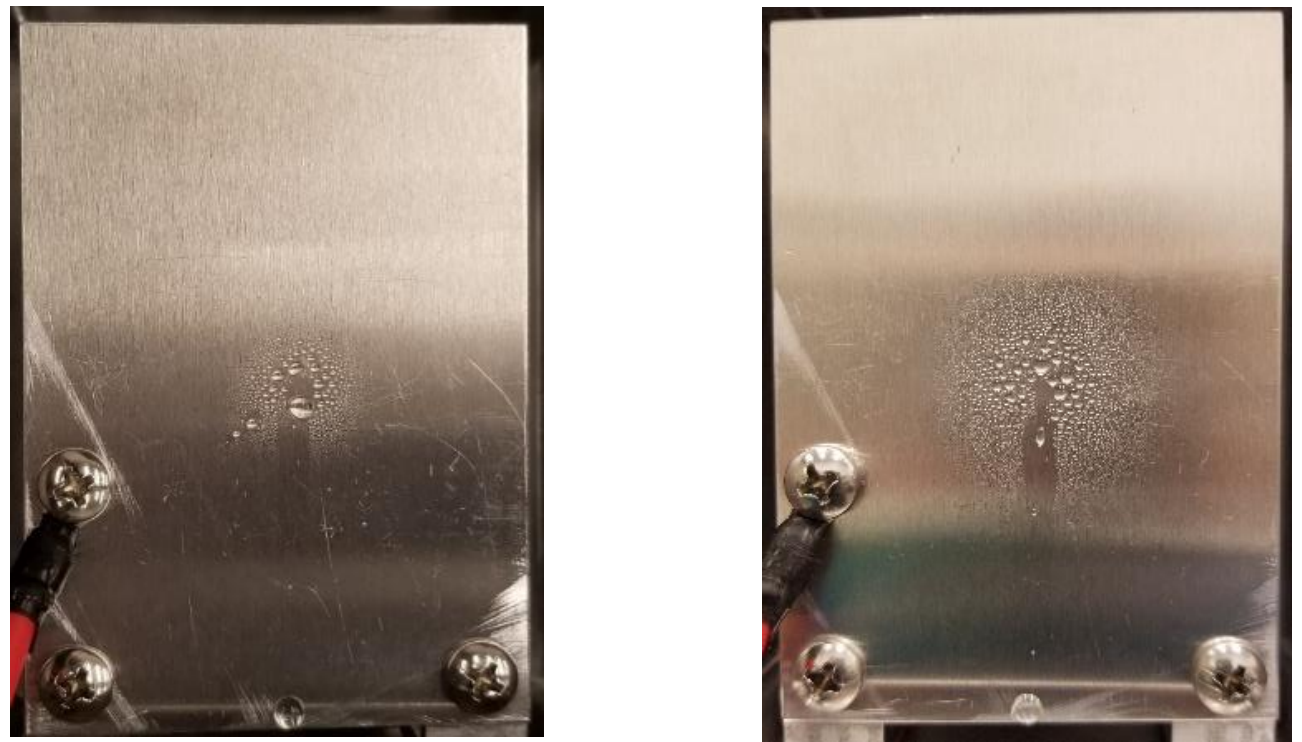

Figure 5.11: Two Examples of the Un-Doped Glycerol Propellant Deposited on the Collector Plate by the Research Thruster After Testing.

Testing using the glycerol doped with sodium iodide went better than with the un-doped glycerol. At all voltages tested, this propellant formed a steady cone-jet. This is undoubtedly due to the increase in electrical conductivity provided by the dopant, as it will respond to an electric field that much more. A conductivity increase of several orders of magnitude was expected, though the values vary greatly depending on the dopant concentration. There does appear to be some minor change in the shape of the emission conejet with changes in the applied voltage. The height of the cone seems to decrease with increasing voltage. This progression is shown in figure 5.12 for an increasing voltage. This is similar to the progression seen with the Demonstration thruster, though not as pronounced likely due to the narrow voltage range of the Research thruster testing.

The collector plate after testing with the doped glycerol looks very much different than those obtained with the un-doped glycerol. A picture of the collector plate can be seen in figure 5.13. There is a similar collection of propellant near the axis of the emitter as expected, however, the emission plume appears to have a significantly greater beam divergence with the doped propellant. This size collector plate and containment box was actually not large enough to collect all of the emissions, meaning a reasonable amount of current was not measured by the pico-ammeter. There is a considerable amount of propellant residue that 
has impacted the lower support metal holding the collection plate assembly, seen at the bottom of figure 5.13. Beyond the noticeable area where the propellant has bound together into large droplets, the pattern is like a fine mist of nearly undiscernible individual droplet size. It is clear after cleaning this plate that the misted area is indeed still liquid and not actually a solid deposition the sodium iodide itself, as was initially speculated upon seeing the results.

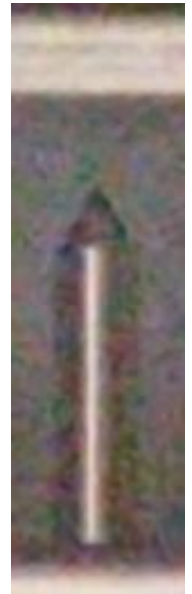

(a)

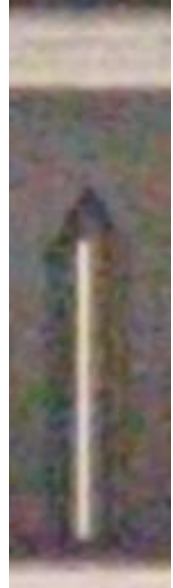

(b)

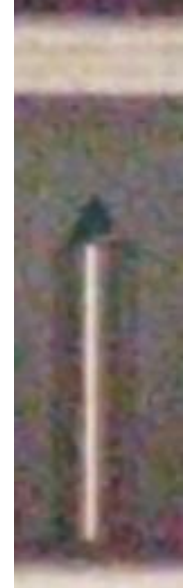

(c)

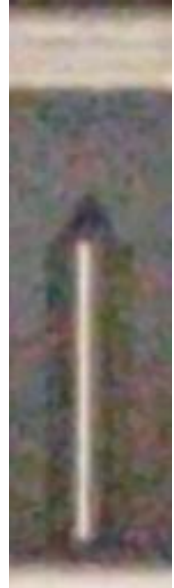

(d)

Figure 5.12: Steady Cone-Jet Formation of the Research Thruster Using Doped Glycerol with the Emitters Charged to Different Voltages: at 4000 V (a), 4150 V (b), 4300 V (c), and 4480 V (d).

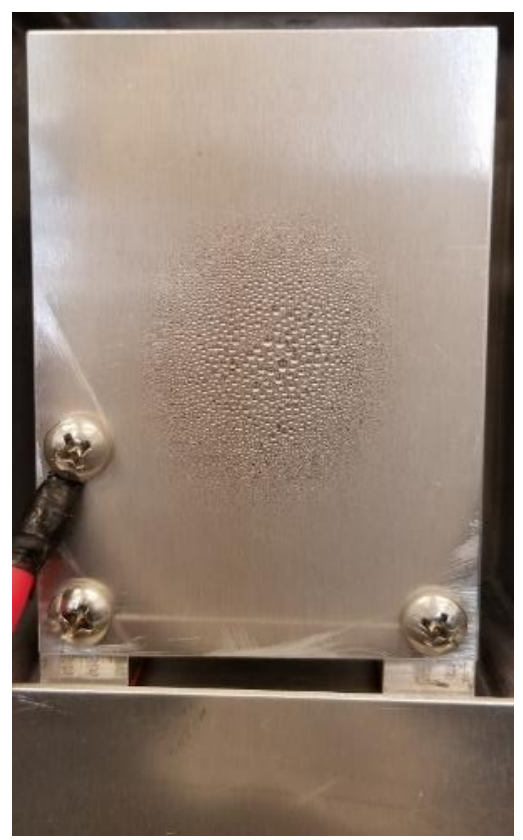

Figure 5.13: Propellant Deposition Pattern on the Collector Plate from Research Thruster Testing Using Doped Glycerol. 


\subsubsection{Data Results}

Data was collected on all of the dry and live tests conducted with the Research thruster at a sample rate of 8 $\mathrm{Hz}$ using the same LabView virtual interface used for the Demonstration thruster testing. The results were analyzed on a qualitative and quantitative basis for comparison between test sessions, and between results when using different propellants. Successful emission is clearly observed in the plotted data through comparison of the dry tests and the live tests. In total, four live tests using un-doped glycerol were conducted (two each when measuring currents from the collection plate and extractor), and one live test using the sodium iodide doped glycerol with current measurements taken from the collection plate.

The dry testing data collected from the Research thruster was used to develop an understanding of the background noise that would be present from the high voltage's presence near the conductive surfaces. Note that this noise is not from the equipment itself, but rather from the charge transfer from the high voltage components to the collection surfaces through the vacuum medium. The raw data shown in figure 5.14 is an example of a dry voltage sweep with current data from the collector plate. The absolute maximum values recorded correspond to the fast changing voltage at the beginning and end of the sweep, and so don't affect the nominal testing scenario. The background noise from ground while at $0 \mathrm{~V} \mathrm{~A}$ is observed to be on the order of $1 \times 10^{-11} \mathrm{~A}$. A clear trend of increasing magnitude in noise is observed with increasing voltage. Currents as high as $5 \times 10^{-10} \mathrm{~A}$ are seen near the times of highest applied voltage of $4500 \mathrm{~V}$. Interesting for this particular example is the periodic oscillation of current when $0 \mathrm{~V}$ is applied. This alludes to a slight disturbance in the common ground used for the testing, potentially caused by ground loops or other currents being drawn nearby in the lab or building.

An example of raw data for a dry test sweep collecting current data from the extractor is given in figure 5.15. The same trend as before is observed: an increasing magnitude of noise as voltage increases. Again, the absolute maximum currents recorded are during the beginning and end of the sweep and a similar minimum at $0 \mathrm{~V}$ applied $1 \times 10^{-11}$. For the noise where the highest voltages are applied, current magnitudes of about $1.5 \times 10^{-9} \mathrm{~A}$ are recorded. This is about an order of magnitude larger than the currents measured from the collector, which is explained primarily because of the proximity of the extractor to the 
charged emitters. Some large spikes in current align with the moments of changing voltage, which is to be expected as it is caused by the changing voltage since the two surfaces act as a capacitor.
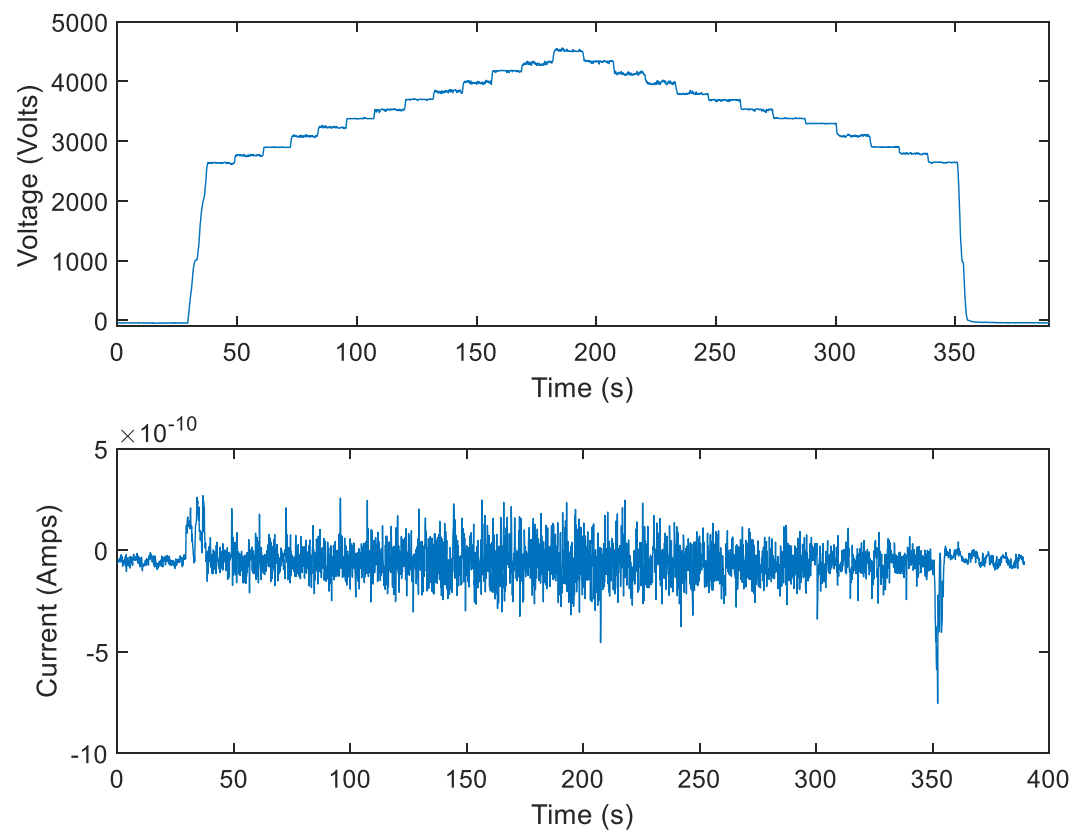

Figure 5.14: Typical Raw Data from the Collector Plate on the Research Thruster During a Dry Voltage Sweep.
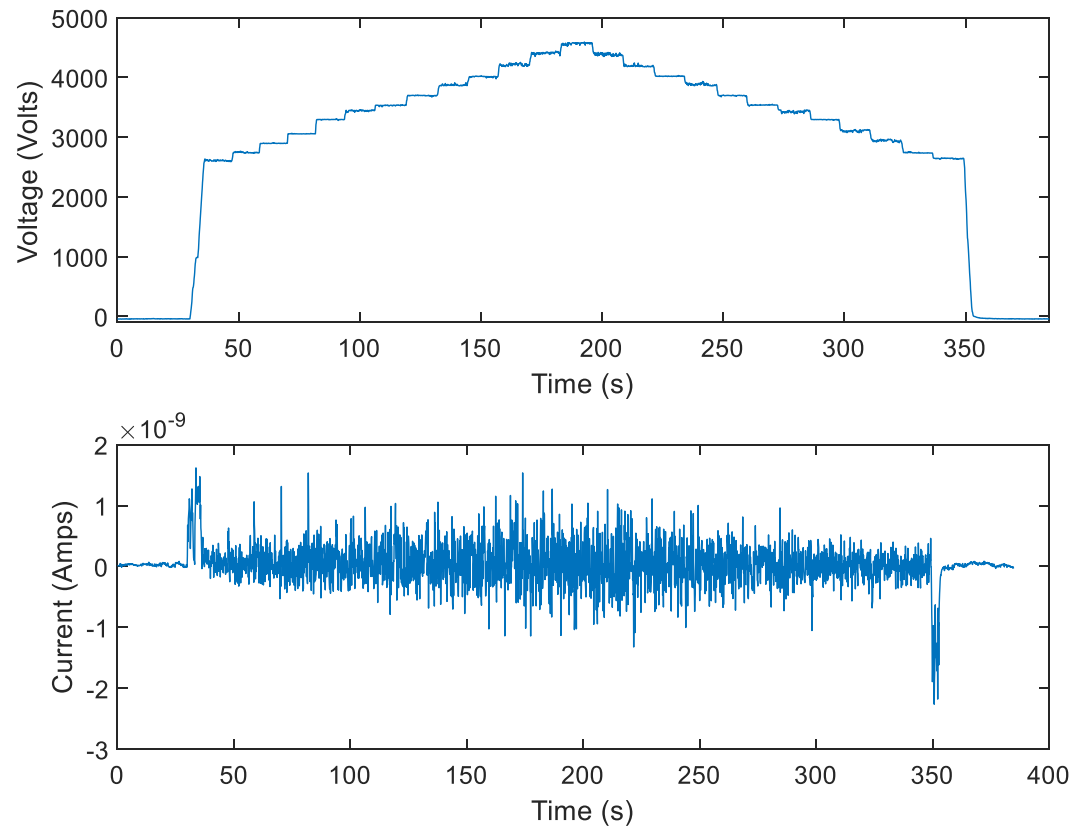

Figure 5.15: Typical Raw Data from the Extractor on the Research Thruster During a Dry Voltage Sweep. 
The noise maximums on the collector plate and the extractor for the Research thruster dry tests are less than half that of the Demonstration thruster dry tests. This reduction happens in spite of several differences in the thrusters that would cause a likely increase in noise, such as the increased areas of the main structure, emitters, and extractor, as well as the higher voltages that are applied to the emitters. These increases are overpowered by the reduction in noise from operating at much lower air pressures, as less air molecules are available to transfer charge from the emitters and main structure to the extractor. Across all of the dry testing with the Research thruster, the measured current magnitudes on the collector plate are within $6.6 \times 10^{-10} \mathrm{~A}$, and the measured current magnitudes on the extractor are within about $1.4 \times 10^{-9} \mathrm{~A}$.

The Research thruster live testing data aligns well with what would be expected after observing the visual results. Since the thruster was emitting in an unsteady cone-jet emission mode, there are frequent peaks of current and the data will shift between these peaks and the background noise which is an order of magnitude less or a greater difference. Figure 5.16 shows the raw data for one of the Research thruster tests measuring current data on the collection plate. Currents as high as $4.9 \times 10^{-8}$ The current spikes are very frequent as the emission is quickly oscillating in the unsteady cone-jet mode, and it doesn't appear to get noticeably less frequent unless there is a disruption altogether. After the initial onset of current, the magnitudes of current quickly decay before settling towards a steady values after several minutes with the emitters at the same voltage, though this is well after the voltage sweep. Several disturbances to point out over the course of the emission, such as the minor arc which is shown by the sudden drop in voltage at about the 150 second mark, resulting in the sudden negative current spike among what is all positive current. The arc could have been caused by a very briefly formed string of propellant bridging the gap between the emitter and the extractor, which is just as quickly vaporized by the relatively high current passed through it. The arcing event is so short and the currents small enough that it was undetectable on video. There is a brief void in collected current around the 470 second mark that is likely caused by a small air bubble that disrupts propellant flow or prevents a cone-jet from forming. The end of the test plotted in figure 5.15 is marked by the severe arcing around the 940 second mark, where the propellant has bridged the gap but is sufficient enough to not be vaporized by the relatively high current that is passed through it. Recall that the power supply is only capable of outputting $4 \mathrm{~mA}$ of current. 

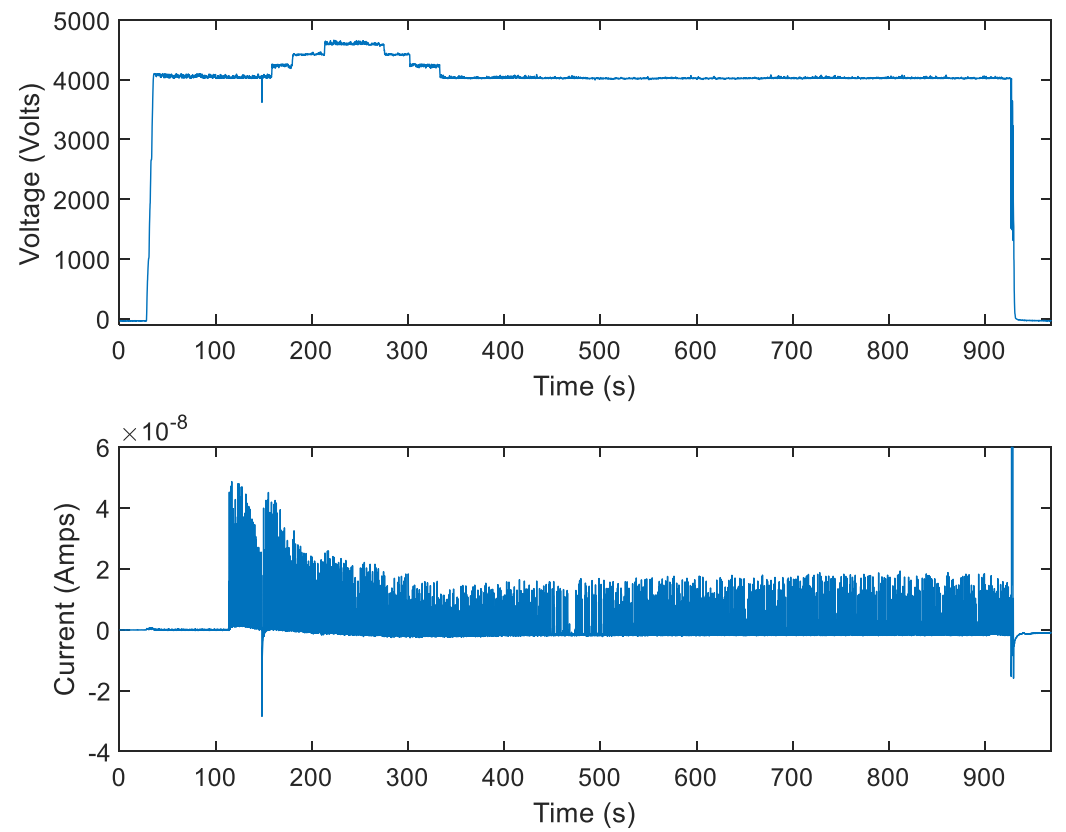

Figure 5.16: Raw Data from the Second Collector Plate Test of the Research Thruster During a Live Voltage Sweep.

Figure 5.17 shows the plots of the raw data corresponding to a test measuring the currents intercepted by the extractor. The positive current spikes are distinct from the noise of the measurements seen from the dry voltage sweep. Note that the intercepted currents are not orders of magnitude greater than the background noise seen during the dry tests. Positive currents as high as $5 \times 10^{-9} \mathrm{~A}$ are measured. It's desirable that the interception be small, but having the measured values so close to the background noise removes the possibility for accurate determination of the average currents, and thus the inefficiencies of the system. The unsteady cone-jet emissions causes the measured current to appear as sharp peaks rather than a consistent value, and the suddenness of the change in charge appears to have some residual effects as even the noise in the measurements increases significantly once emission has begun. More than just noise, the negative currents may indicate a flow of charge in the opposite direction intended, where propellant is emitting from the extractor onto the emitters and main structure. There is certainly enough surface area between the two where the electric field may be strong enough to achieve emission from the deposited propellant on the extractor. Over time, this may cause increased chance of thruster failure and reduced performance as more propellant is deposited on the extractor and affects the trajectory of newly accelerated droplets. Also notice 
the minor arc that took place around the 230 second mark, though this doesn't appear to have caused the same response in the current measurements that was seen from the arc during the collector tests.
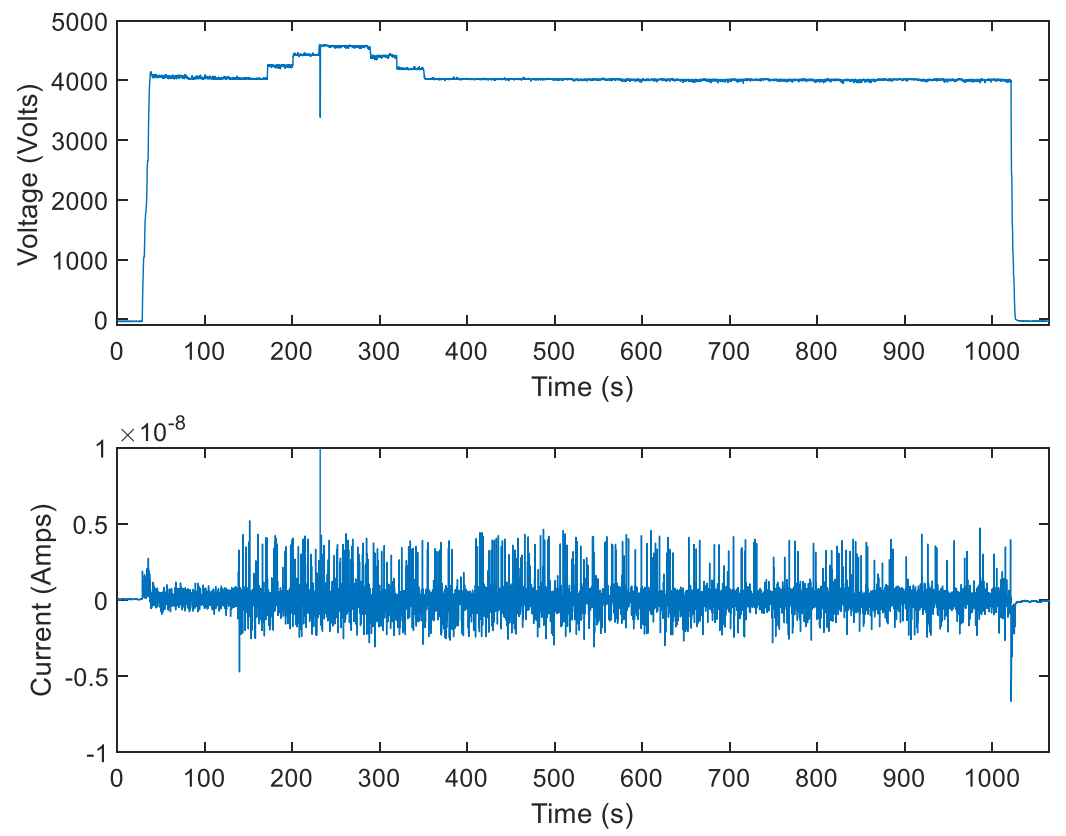

Figure 5.17: Raw Data from the Second Extractor Test of the Research Thruster During a Live Voltage Sweep.

The two tests on each of the collection surfaces showed reasonable repeatability for the Research thruster when using un-doped glycerol propellant. For the collector plate tests, the current magnitude is greatest near the first onset of emission, near $4.9 \times 10^{-8} \mathrm{~A}$, and decays over time to settle near about $1.8 \times 10^{-8}$ A. Tests measuring current on the extractor recorded consistent currents throughout the sweep near about $4 \times 10^{-9}$ A. Assuming that the entirety of the glycerol emissions are captured by either the collection plate or the extractor, the relative magnitudes in current shows that almost $17 \%$ of the current is being intercepted by the extractor in some cases. Though this is a rough estimate using data from two completely separated test sessions, it is an indication of the performance of this design as a whole.

Table 5.1 displays the calculated averages for the current measurements at each of the voltage steps in the sweep for the data of the second live test measuring current on the collector plate. This test data was chosen to for further analysis due to its more consistent operation over time. The second live test measuring the current intercepted by the extractor, shown in table 5.2, is chosen for the same reasons. The time average of the current does a poor job at representing the thruster's performance, since the thruster is emitting in an 
unsteady cone-jet which oscillates between high and no currents being measured and thus returns an average with a high standard deviation. As such, an average of only the peaks in current was included as well. This only takes into account the current measurements that are above a magnitude threshold set by the highest magnitudes of noise seen during the relevant dry voltage sweep tests, and so provides a better picture of the actual emission.

Table 5.1: Voltages and Corresponding Current Values from the Second Collector Plate Test of the Research Thruster Using Un-doped Glycerol Propellant.

\begin{tabular}{|l|l|l|}
\hline Voltage (V) & Time Average Current (nA) & Average Peak Current (nA) \\
\hline $4040.2+/-27.7$ & $7.205+/-13.40$ & $22.96+/-14.67$ \\
\hline $4233.7+/-17.7$ & $5.544+/-11.20$ & $21.68+/-12.00$ \\
\hline $4422.8+/-7.80$ & $3.974+/-8.989$ & $18.56+/-7.425$ \\
\hline $4601.2+/-17.6$ & $2.747+/-7.804$ & $15.31+/-5.964$ \\
\hline $4421.6+/-6.13$ & $0.8272+/-5.716$ & $11.37+/-3.995$ \\
\hline $4237.2+/-16.5$ & $0.4434+/-5.005$ & $9.096+/-4.206$ \\
\hline $4031.2+/-12.2$ & $0.3044+/-4.634$ & $8.500+/-3.534$ \\
\hline
\end{tabular}

Table 5.2: Voltages and Corresponding Current Values from the Second Extractor Test of the Research Thruster Using Un-doped Glycerol Propellant.

\begin{tabular}{|l|l|l|}
\hline Voltage (V) & Time Average Current (nA) & Average Peak Current (nA) \\
\hline $4026.8+/-7.35$ & $0.05233+/-1.103$ & $3.447+/-0.9765$ \\
\hline $4242.8+/-14.4$ & $0.1560+/-0.9441$ & $3.490+/-0.5799$ \\
\hline $4427.0+/-9.15$ & $0.09185+/-0.9847$ & $2.941+/-0.8186$ \\
\hline $4572.1+/-13.7$ & $0.1659+/-1.068$ & $3.058+/-0.6795$ \\
\hline $4401.9+/-19.0$ & $0.005874+/-0.9046$ & $3.008+/-0.5179$ \\
\hline $4190.6+/-9.91$ & $0.04492+/-0.9656$ & $3.128+/-0.6056$ \\
\hline $4020.7+/-4.99$ & $-0.001671+/-1.085$ & $3.251+/-0.5792$ \\
\hline
\end{tabular}

The data for the time average currents for the collector current data from table 5.1 is plotted in figure 5.18. Due to the large standard deviation error in the data, it is impossible to declare a trend in the relation of the voltage applied and the currents measured. Again, including the data points where the thruster is not emitting skews the average and the error calculations. The average of the emission peaks is plotted in figure 5.19 and shows a slightly improved picture compared to the time average. Though the error on the increasing side of the voltage sweep is still large, the error does consistently decrease as the voltage sweep continues. As the thruster continues to operate, it steadily approaches an equilibrium over time. The time spent on the voltage sweep does not accurately capture this process, as only a total of 60 seconds are spent at each voltage. 


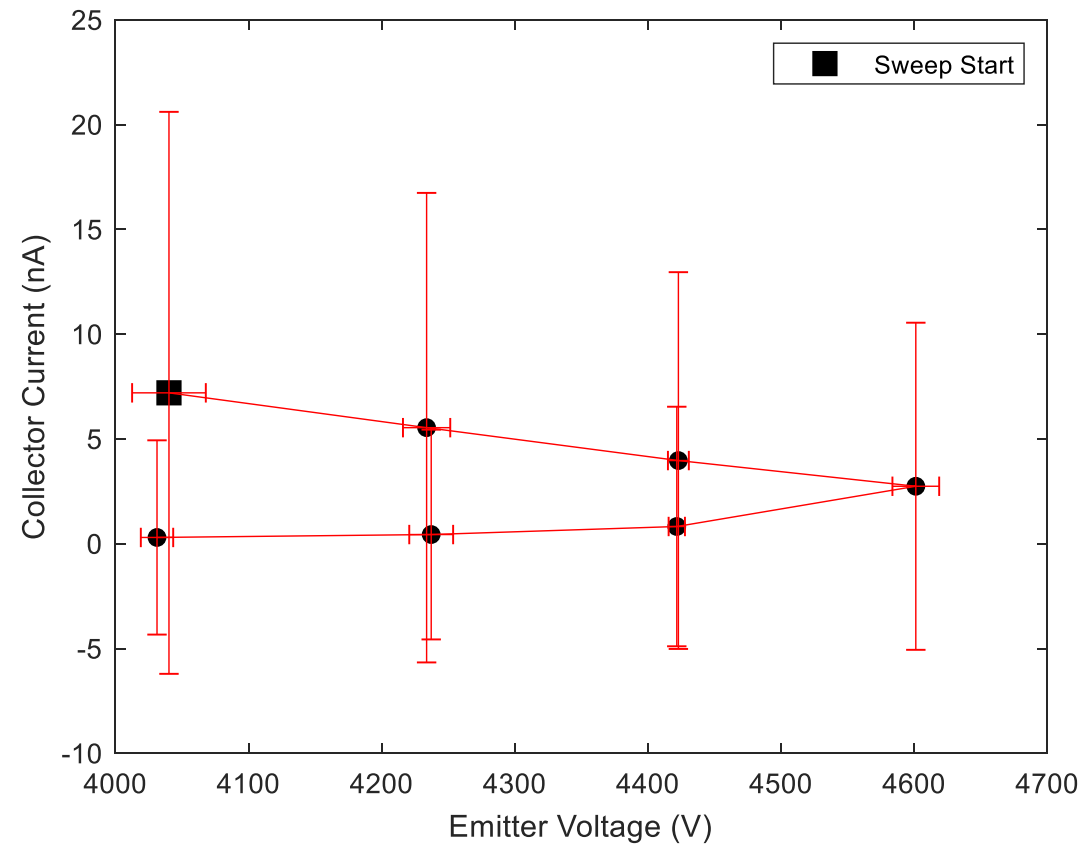

Figure 5.18: IV Trace Values Averaged Over Time for the Second Collector Plate Test of the Research Thruster Using Un-Doped Glycerol Propellant.

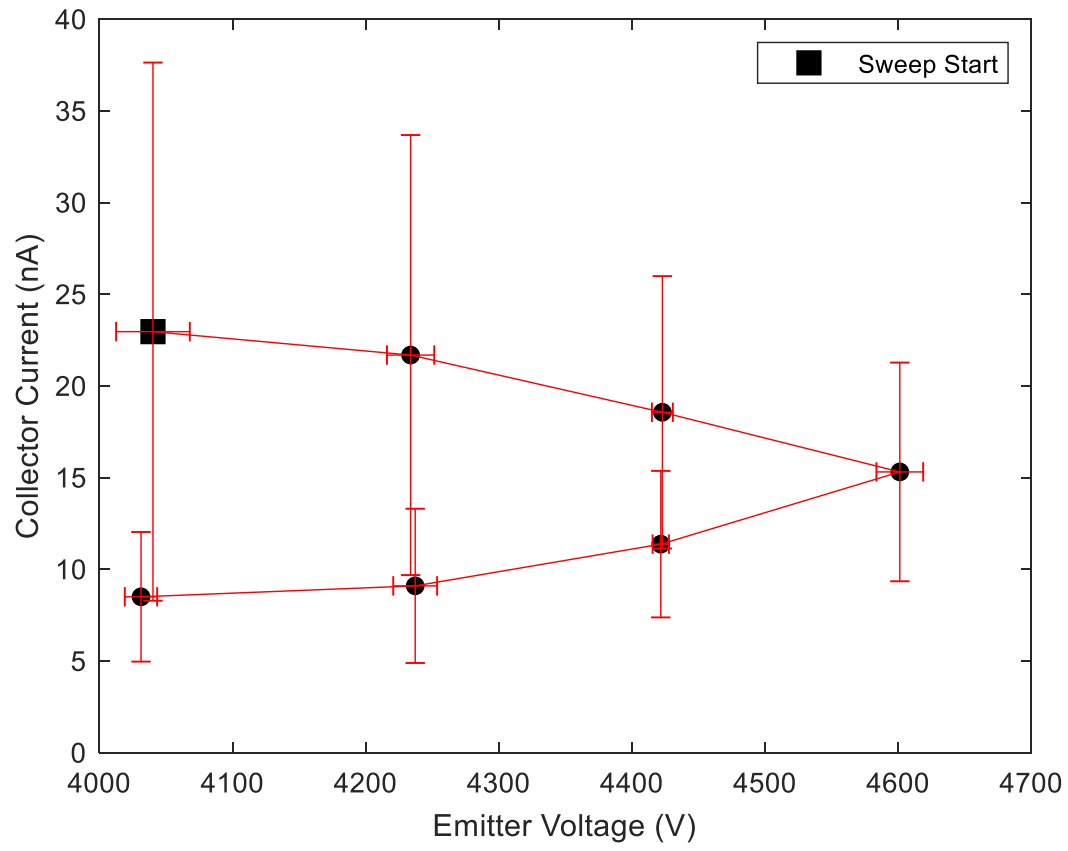

Figure 5.19: IV Trace Values Averaged Across the Peaks in Current for the Second Collector Plate Test of the Research Thruster Using Un-Doped Glycerol Propellant.

The data for the time average currents for the collector current data from table 5.1 is plotted in figure 5.20. As with the collector plate data, it is impossible to make any claims about any trends in the data due to 
the error associated with the values, though it's clear why this error is present. An unsteady cone-jet emission oscillates between the background noise and a higher current measurement during the actual emission, skewing the average and creating a large standard deviation. The average of the emission peaks is plotted in figure 5.21 and shows the expected change from excluding the portions of the data lacking emission. The average is not obviously positive though the error still prevents any worthwhile discussion.

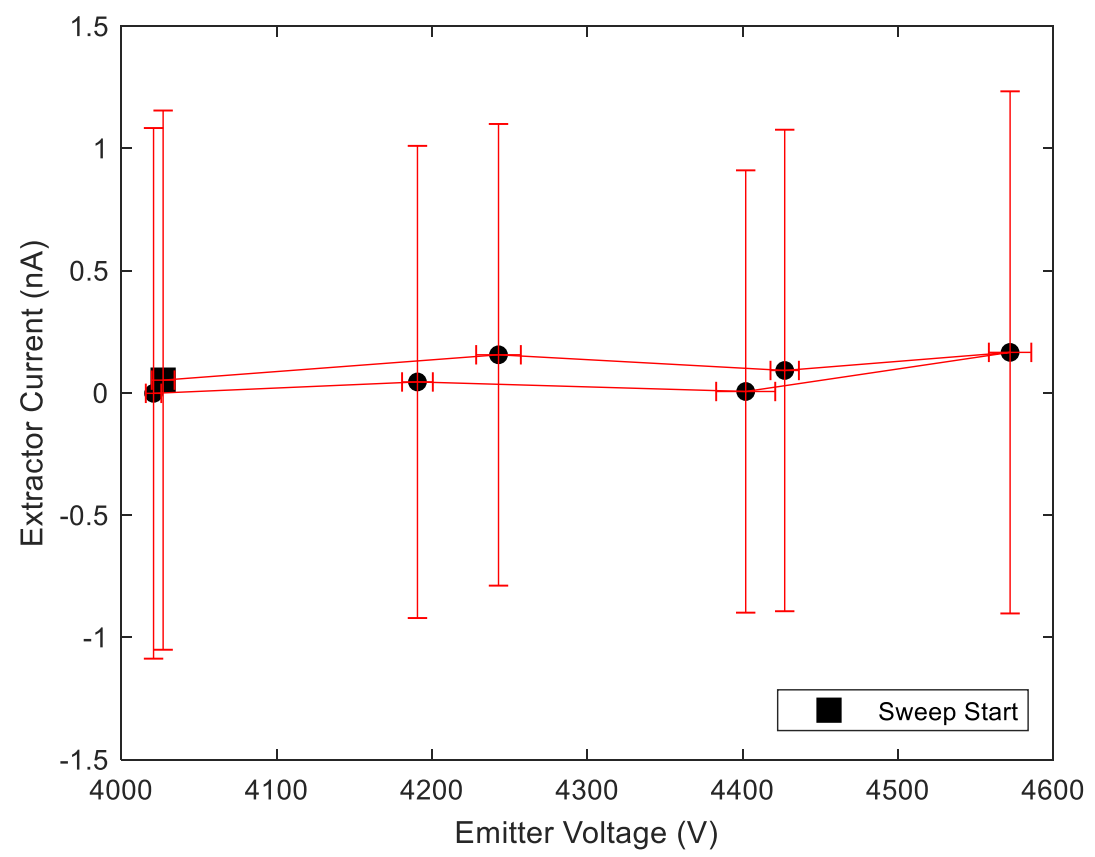

Figure 5.20: IV Trace Values Averaged Over Time for the Second Extractor Test of the Research Thruster Using Un-Doped Glycerol Propellant.

The raw data for the current measurements on the collector plate using sodium iodide doped glycerol are plotted in figure 5.21. Note that the data frequently goes out of the current sensing range of the picoammeter during this test, and so the equipment software returns a very large quantity to represent infinity, depicted by the vertical lines stretching above the other data points on the plot in same manner as the arcing seen on other data sets. Changing the range on the pico-ammeter would cause an increase in the background noise of the measured current by several orders of magnitude, and so it was deemed not necessary for the purpose of this testing. 


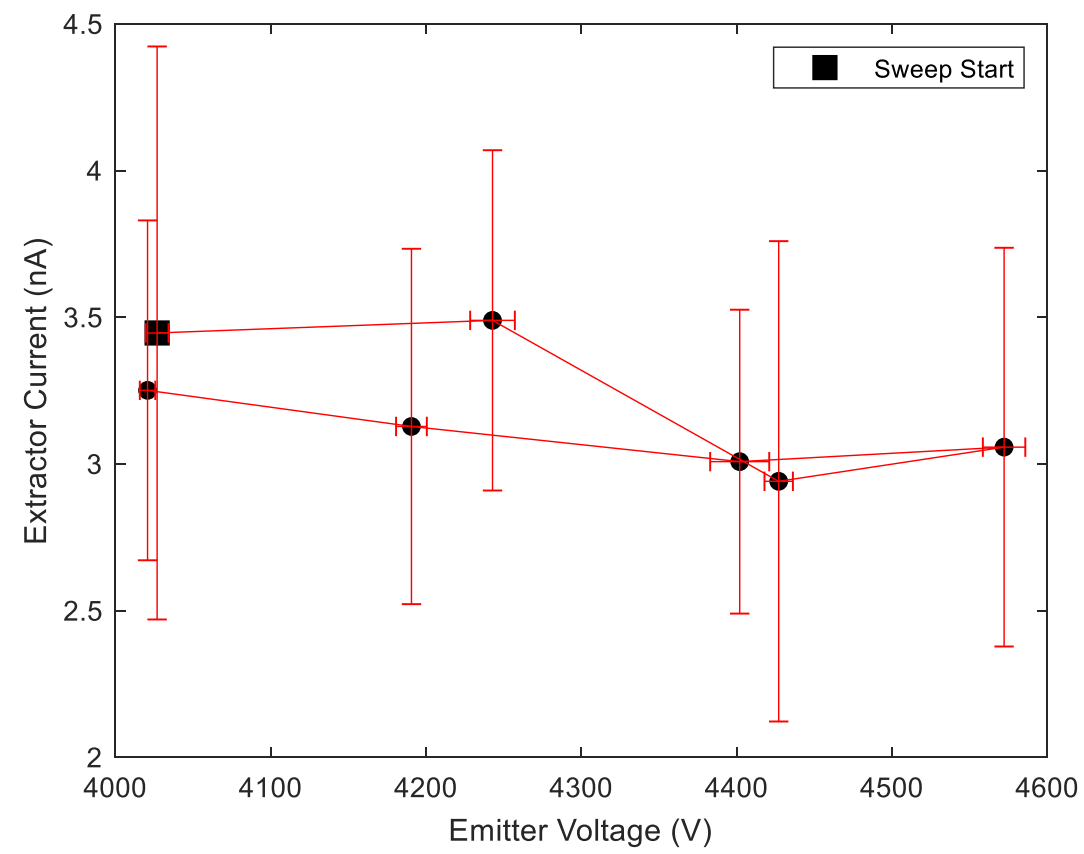

Figure 5.21: IV Trace Values Averaged Across the Peaks in Current for the Second Extractor Test of the Research Thruster Using Un-Doped Glycerol Propellant.

There is an increased frequency of minor arcing throughout the testing using the sodium iodide doped glycerol compared to the un-doped glycerol tests, which is indicated by a sharp drop in voltage and current in the respective plots. The frequency and isolation of these arcing events did not cause enough concern to abort the testing, and the events did not seem to significantly alter the data gathered. Current measurements wander across this range over time, and it's difficult to tell from the raw data whether or not the currents extracted are significantly affected by the applied voltage, as would be expected. Even past the voltage sweep, the currents continue to shift periodically, seemingly affected by some other primary influence. This influence is likely the amount of propellant that is deposited on the extractor rather than continuing to the collection plate, which was not measured for this propellant. If there are large amounts of propellant accumulating on the extractor, it would increase the intercepted current as the trajectory of the newly accelerated droplets is affected and more likely to be attracted to the extractor and the already deposited propellant. Other than the changing electric field lines due to this deposition, there is a high likelihood that the cross section of the extractor hole would be decreased as propellant builds up on the extractor. As this accumulation changes shape and moves as more propellant accumulates, there would be a somewhat unpredictable effect on the current able to pass through the extractor to the collector plate. If the obstructions 
happens to change in such a way that more current is allowed through, then that would be seen as an increase in current measured on the collector despite no voltage change by the operator. This is exactly what is seen with the raw data from this test.

The raw data for the current measurements on the collector plate using sodium iodide doped glycerol are plotted in figure 5.22. Note that the data frequently goes out of the current sensing range of the picoammeter during this test, and so the equipment software returns a very large quantity to represent infinity, depicted by the vertical lines stretching above the other data points on the plot in same manner as the arcing seen on other data sets. Changing the range on the pico-ammeter would cause an increase in the background noise of the measured current by several orders of magnitude, and so it was deemed not necessary for the purpose of this testing. There is an increased frequency of the minor arcing throughout the voltage sweep compared to the un-doped glycerol tests, which is indicated by a sharp drop in voltage and current in the respective plots. The frequency and isolation of these arcing events did not cause enough concern to abort the testing, and the events did not seem to significantly alter the data gathered. Current measurements wander across this range over time, and it's difficult to tell from the raw data whether or not the currents extracted are significantly affected by the applied voltage, as would be expected. Even past the voltage sweep, the currents continue to shift periodically, seemingly affected by some other primary influence. This influence is likely the amount of propellant that is deposited on the extractor rather than continuing to the collection plate, which was not measured for this propellant. If there are large amounts of propellant accumulating on the extractor, it would increase the intercepted current as the trajectory of the newly accelerated droplets is affected and more likely to be attracted to the extractor and the already deposited propellant. Other than the changing electric field lines due to this deposition, there is a high likelihood that the cross section of the extractor hole would be decreased as propellant builds up on the extractor. As this accumulation changes shape and moves as more propellant accumulates, there would be a somewhat unpredictable effect on the current able to pass through the extractor to the collector plate. If the obstructions happens to change in such a way that more current is allowed through, then that would be seen as an increase in current measured on the collector despite no voltage change by the operator. This is exactly what is seen with the raw data from this test. 

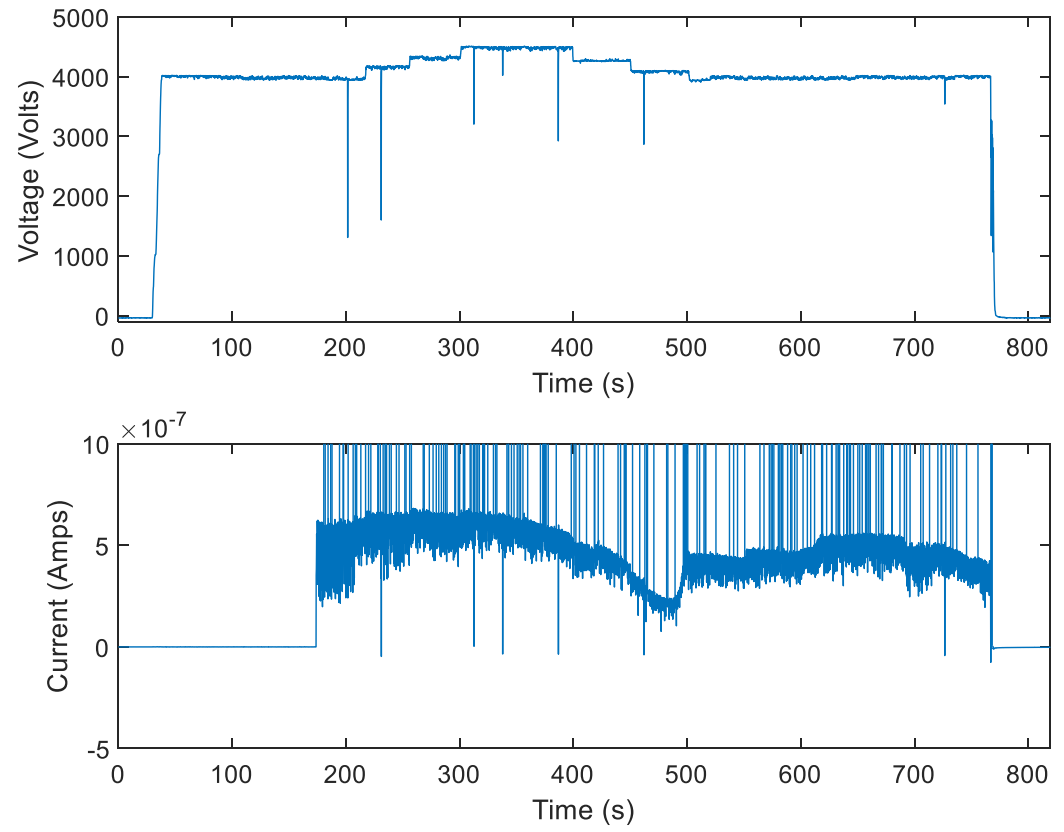

Figure 5.22: Raw Data from the Collector Plate of the Research Thruster During a Live Voltage Sweep Using Sodium Iodide Doped Glycerol Propellant.

Table 5.3 displays the calculated averages for the current measurements at each of the voltage steps in the sweep for the data of the live test measuring current on the collector plate. As the thruster was operating in a steady cone-jet mode, and the current was not oscillating, the time average is also the peak average as all values surpass the threshold set by the background noise seen in the dry testing.

Table 5.3 Voltages and Corresponding Current Values from the Collector Plate Test of the Research Thruster Using Sodium Iodide Doped Glycerol Propellant.

\begin{tabular}{|l|l|}
\hline Voltage (V) & Current (nA) \\
\hline $3961.2+/-25.5$ & $497.2+/-121.9$ \\
\hline $4157.3+/-28.7$ & $573.0+/-89.17$ \\
\hline $4313.0+/-20.5$ & $597.1+/-67.84$ \\
\hline $4484.2+/-26.9$ & $571.8+/-74.34$ \\
\hline $4266.3+/-8.24$ & $443.0+/-63.91$ \\
\hline $4085.7+/-17.6$ & $281.5+/-100.6$ \\
\hline $3983.9+/-19.6$ & $405.2+/-59.06$ \\
\hline
\end{tabular}

The data for the time average currents for the collector current data from table 5.3 is plotted in figure 5.23. The error associated with the average values is much more manageable compared to the test using undoped glycerol, though the trend in current is not what would be expected, as discussed with the raw data, there is clearly some other primary influence that is affecting the current imparted on the collection plate rather than the voltage applied to the emitters. Over a voltage sweep, it is expected that the extracted current 
will increase as voltage increase. This trend is seen for the beginning of the increasing half of the sweep, but at the highest voltage tested, the current starts to decrease due to other influences previously discussed.

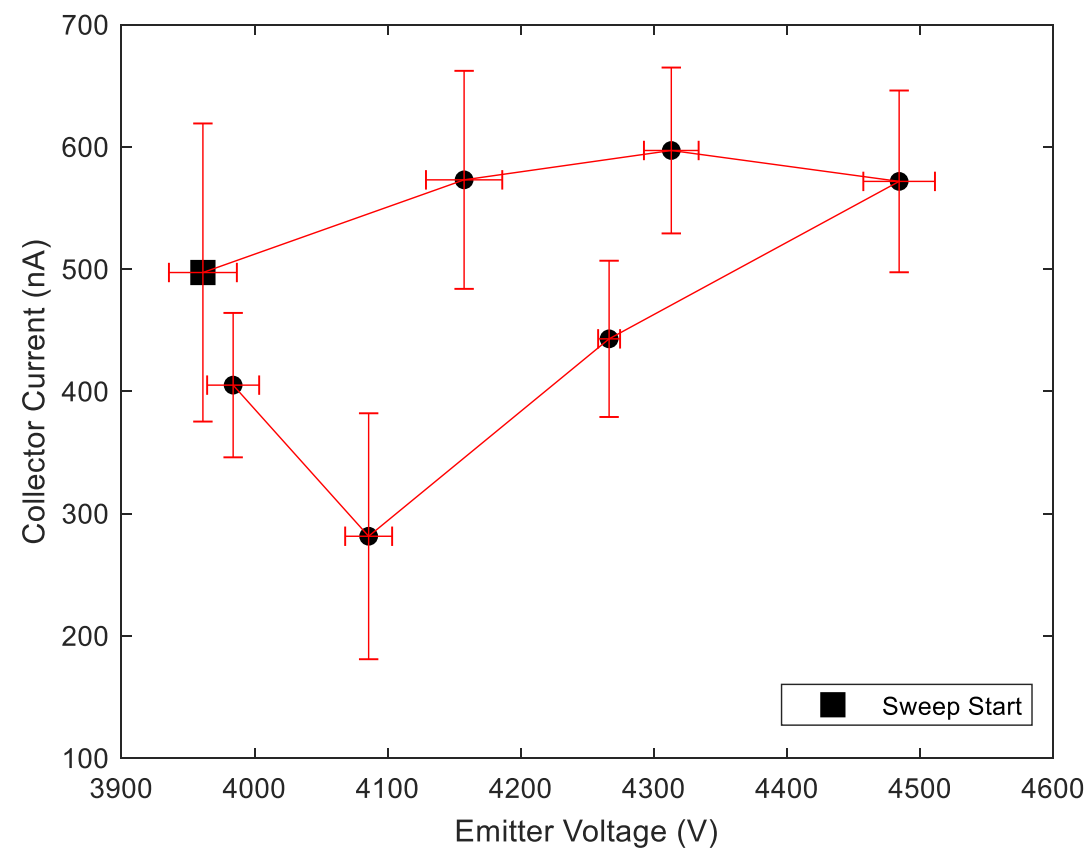

Figure 5.23: IV Trace Values Averaged Over Time for the Collector Plate Test of the Research Thruster Using Sodium Iodide Doped Glycerol Propellant.

\subsection{Discussion}

While the testing of the Research thruster was successful in the goal of achieving quantified emission using two propellants, there is much room for improvement and some known errors that can corrected through continued design iterations. There were several assumptions in the design that did not hold past testing with un-doped, and the addition of the dopant caused some unexpected results.

Some interception of the emissions by the extractor is expected, as it is unavoidable that some particles are attracted to the extractor. Inefficiencies like this are common throughout the field, as explained by R. Wirz's presentation on the subject of electrosprays in May 2019 [29]. Though when the concept of interception is thought of, the surface of the extractor facing the emitters usually is the initial thought. What was observed throughout the testing in this thesis is the recirculation of propellant particles that escape through the extractor but return to deposit themselves onto the outward facing surface. Some examples of 
this phenomenon after various tests are shown in figure 5.24. It's interesting to note that the Research thruster testing where current data was collected from the extractor had essentially no propellant on the outside face of the extractor. This is likely for reasons similar to how the emissions of the Demonstration thruster were greatly stunted by having the pico-ammeter connected to the extractor. While this sort of deposition may not have a significant effect towards causing the thruster to fail, it certainly contributes to losses in the instantaneous performance of the thruster. Coupled with the fact that some emissions are not collected by either the collection plate or the extractor, accurate performance calculations are unobtainable.

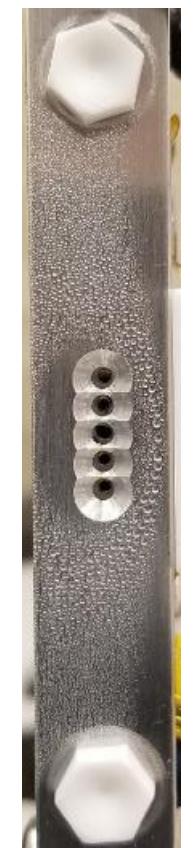

(a)

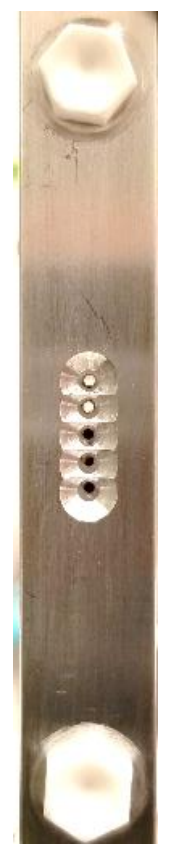

(b)

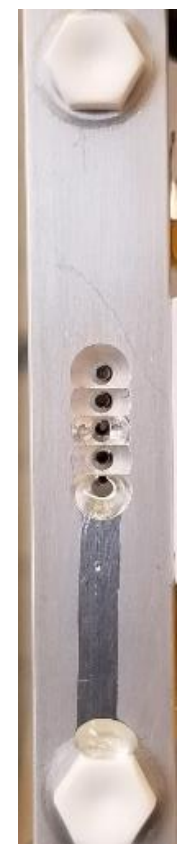

(c)

Figure 5.24: Propellant Deposition on the Outside Face of the Extractor on the Research Thruster After the Second Collector Plate Test Using Un-Doped Glycerol (a), the Second Extractor Test Using Un-Doped Glycerol (b), and the Collector Plate Test Using Sodium Iodide Doped Glycerol (c).

Intermittent arcing also played a role in affecting the data enough so that accurate calculations were difficult. This manifested as invisible breakdown that would cause the pico-ammeter to have erroneous current output for several seconds or more. Though they don't cause any failures of the thruster, it still affects the performance significantly. The charge of these arcs is likely traveling a path that jumps along many droplets of propellant as it travels near and through the emitter. There is no clear way to combat this directly, though systems with different dimensions and designs may be less likely to endure events such as these. 
The most common failure mode for the Research thruster is a bridge of propellant shorting the gap between the emitter and the extractor, causing repeated arcing through the propellant. Figure 5.25 shows an example of the severity of the arcing the can occur. Though the current delivered by the power supply is 4 $\mathrm{mA}$, it's not safe for arcing to occur if the pico-ammeter is connected, as damage may occur. When this arcing is observed the test was aborted immediately.

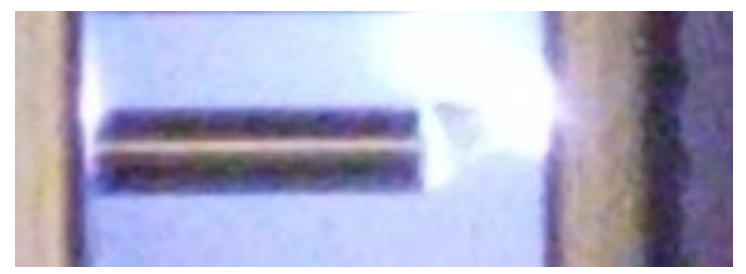

Figure 5.25: Example of Electrical Arcing During a Failure of the Research Thruster Where Propellant Had Bridged the Gap Between the Emitters and the Extractor.

An effort was made throughout this thesis to observe any physical degradation as a result of the thruster's operation over time. Microscope images of the emitters and extractors were taken before and after the testing was conducted, though no visible changes are apparent at 80 times magnification. These images are shown in figure 5.26. The research avenue of characterizing the degradation of an electrospray would likely involve work going well beyond that of this thesis.

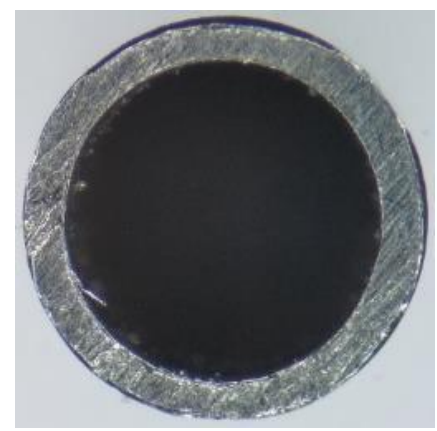

(a)

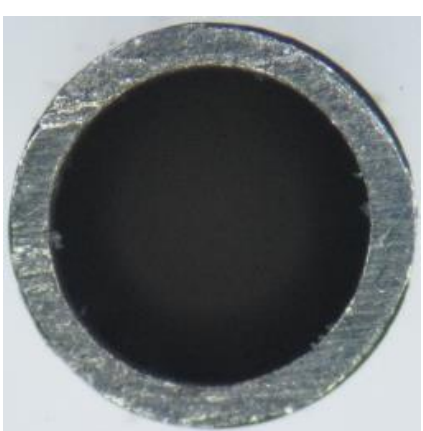

(b)

Figure 5.26: Comparison Between Before and After Testing of the Needle End on the Most Used Research Thruster Emitter Needle. 


\section{Chapter 6}

\section{CONCLUSION}

Overall, the work conducted in this thesis was successful in designing and testing electrospray thrusters which perform well for their intended purposes. The Demonstration thruster served well to easily provide visualization of an operating electrospray thruster, and the Research thruster was shown to have value for additional testing and research with electrosprays. With any project, there are many avenues for improvement based on the lessons learned from its completion.

\subsection{Conclusions}

Two designs of electrospray thruster were fabricated and tested to confirm their successful operation. The Demonstration thruster design is tailored towards visualization of the core concepts of an electrospray, while the Research thruster design aims to become a platform in which more rigorous research may be conducted. A simple diagnostic technique using a pico-ammeter to directly measure the current incident on a collection plate sized to collect all of the emitted propellant, or the extraction grid on the thrusters themselves. Measured currents are plotted against the voltage applied to the emitters to determine any relationships between the two.

The Demonstration thruster served its purpose of a basic representation of an electrospray thruster and the physical phenomena present during their operation, though the data collection is muddled by interference from the atmosphere. Current measurements taken from the collection plate and extractor that reveal successful emission are sparse and inconsistent due to the charged droplets' interaction with the high density of air molecules in atmospheric conditions. When successful measurements were made, the maximum currents recorded were $3.8 \times 10^{-9} \mathrm{~A}$ on the collection plate and $9.7 \times 10^{-8} \mathrm{~A}$ on the extractor, where the emitter voltage was swept from $2100 \mathrm{~V}$ to $3700 \mathrm{~V}$. The difference illustrates how much charge on the propellant is lost as it travels through the air.

Research thruster testing using un-dope glycerol gave currents as high as $4 \times 10^{-8} \mathrm{~A}$ on the collection plate and $5 \times 10^{-9} \mathrm{~A}$ on the extractor when emitter voltage was swept from $4000 \mathrm{~V}$ to $4500 \mathrm{~V}$ for a single 
emitter setup. Single emitter testing using glycerol doped with sodium iodide at $20 \%$ by weight gave currents as high as $7 \times 10^{-7} \mathrm{~A}$ on the collection plate. Using un-doped glycerol as propellant, comparing currents measured on the extractor versus the collection plate reveal an interception rate as high as $17 \%$ of the current extracted from the emitter. The testing confirmed that the Research thruster is capable of operating as an electrospray worth further study and application. Some changes to the testing diagnostic setup would be beneficial to testing, such as an additional pico-ammeter to measure currents on the collection plate and extractor simultaneously. Additional design work is necessary to achieve reliable multi-emitter emission.

There are clear paths to take beyond the work done in this thesis, which serves as only a starting point for electrospray research efforts at Cal Poly, and the Demonstration thruster and Research thruster are well suited to that end due their accessibility and robustness.

\subsection{Future Work}

The work completed in this thesis is the beginning efforts of electrospray research efforts at Cal Poly, and its legacy is as much the physical thrusters as it is the first-hand operational knowledge gained only by repeated efforts to create a properly functioning electrospray thruster. Many opportunities for future work are presented as a result of the successes and short comings of this electrospray research. There are some basic modifications to the Research thruster that are necessary to improve the function of the system as a whole, such as increasing the size of the collector plate so that all of the emissions are captured for all propellant choices. More rigorous testing of the Research thruster would be necessary to better define its performance, as well as additional diagnostics that can characterize different aspects of an electrosprays performance.

Foremost is the need for more or better electrical equipment. The glassman power supply used in this thesis is very old and so does not have many of the inputs and outputs that make testing much more centralized and simple, such as serial port or any communication port that can be connected to a computer. Only having the one pico-ammeter throughout the course of this testing certainly increased the complexity of the testing procedure as it essentially doubled the testing sittings that needed to be completed. More than operational concerns, it prevented any accurate comparison of the extractor and collector currents, as these currents were recorded during separate instances of thruster operation. 
Additional work that doesn't require much modification or increased depth of analysis is to use the thruster in its current state with a wider range of propellant types. Best suited for this are other chemical dopants for glycerol, of which there are many to choose from that have been used in previous research with colloids. Another simple change that can be made is the application of smaller capillary emitters, which would become closer in size to those used currently in research, though more advanced methods of the video capturing would be necessary to see the cone-jet formation well enough. Other worthy propellants such as ionic liquids may require more modification to the existing system to be successful. An attempt was made to use ionic liquid EMI-BF4 as part of this thesis, but the reduced viscosity and surface tension relative to glycerol created some interesting effects in the propellant feed lines that prevented any prolonged testing from being conducted.

A fair amount of testing was dedicated to having uniform emission across a linear array of five emitters, though success in this endeavor was not fully realized. A focused look could be taken into how to achieve uniform flow across the five emitters present currently, or any number of multiple emitters. It would be beneficial from an operational and monetary perspective to avoid simply having five separate propellant feed lines and syringes. A brute force approach like this would require additional feedthroughs and more capable pumping equipment.

An important aspect of electrospray research at Cal Poly is what can be reasonably accomplished with the facilities available, both using this thesis work and beyond it. Furthermore, research that can contribute to the electrospray community must be pursued. An example of a potential avenue is thruster lifetime, exploring how components degrade over time from thruster operation. Adaptations to the Research thruster can be made to accommodate this kind of testing, though other equipment would be necessary for adequate analysis of components, such as optical microscopes and the like. The simple design of the Research thruster's components allows for quick machining and replacement, which would be often necessary when working with degraded parts. 


\section{BIBLIOGRAPHY}

[1] D. N. Baker, and S. P. Worden, The Large Benefits of Small Satellite Missions, EOS, Vol. 89, pp. 301-312, 2008.

[2] NASA, COTS Components in Spacecraft Systems: Understanding the Risk, Technical Update, NASA, 2014, https://www.nasa.gov/sites/default/files/atoms/files/cots.pdf [Accessed July 2019].

[3] CubeSat, Developer Resources, http://www.cubesat.org/ [Accessed July 2019].

[4] M. M. Micci and A. D. Ketsdever, Micropropulsion for Small Spacecraft, Progress in Astronautics and Aeronautics, Vol. 187, AIAA, 2000.

[5] MIT OpenCourseWare, Session 20: Electrospray Propulsion, https://ocw.mit.edu/courses/ aeronautics-and-astronautics/16-522-space-propulsion-spring2015/lecturenotes/ MIT16_522S15_Lecture20.pdf [Accessed June 2019].

[6] C. Ma, and C. N. Ryan, The Design and Characterization of a Porous-emitter Electrospray Thruster (PET-100) for Interplanetary CubeSats, 7th Interplanetary CubeSat Workshop May 2930, iCubeSat, Paris, France, 2018.

[7] G. P. Sutton, and O. Biblarz, Rocket Propulsion Elements, Eighth Edition, Wiley, Hoboken, NJ, 2010.

[8] R. L. Grimm, Fundamental Studies of the Mechanisms and Applications of Field-Induced Droplet Ionization Mass Spectrometry and Electrospray Mass Spectrometry, PhD Thesis, California Institute of Technology, 2006.

[9] J. Ziemer, et al., Colloid Microthruster Flight Performance Results from Space Technology 7 Disturbance Reduction System, 35th International Electric Propulsion Conference October 8-12, Georgia Institute of Technology, GA, 2017.

[10] D. Krejci, et al., Demonstration of the IFM Nano FEEP Thruster in Low Earth Orbit, 4S Symposium May 28-June 1, Sorrento, Italy, 2018.

[11] J. Rosell-Llompart, J. Grifoll, and I. G. Loscertales, Electrosprays in the cone-jet mode: From Taylor cone formation to spray development, Journal of Aerosol Science, Vol. 125, 2018.

[12] T. Morris, C. Malardier-Jugroot, and M. Jugroot, Characterization of electrospray beams for micro-spacecraft electric propulsion applications, Journal of Electrostatics, Vol. 71, 2013.

[13] S. Verdoold, , L. L. F. Agostinho, C. U. Yurteri, and J. C. M. Marijnissen, A generic electrospray classification. Journal of Aerosol Science, Vol. 67, pp. 87-103, 2014.

[14] F. M. Pranajaya, and M. A. Cappelli, Performance Studies of a Colloid Thruster System, 27 International Electric Propulsion Conference October 15-19, Pasadena, CA, 2001.

[15] A. M. Ralph, A. G. Hsu, J. A. Young, and T. J. Curtiss, Characterization of a Single Emitter Electrospray Thruster, Technical Report, Aerospace Corp., 2016. 
[16] H. Dastourani, M. R. Jahanama, A. Eslami-Majd, A physical insight into electrospray process in cone-jet mode: Role of operating parameters, International Journal of Heat and Fluid Flow, Vol. 70, 2018.

[17] Busek Co., Inc., Electrospray Thrusters, http://www.busek.com/technologies_espray.htm [Accessed June 2019].

[18] Accion Systems Inc., Tiled Ionic Liquid Electrospray, https://www.accion-systems.com/tile [Accessed June 2019].

[19] NASA JPL, Electrospray Propulsion, https://microdevices.jpl.nasa.gov/capabilities/advancedmicrofabrication-technologies/electrospray-propulsion/ [Accessed June 2019].

[20] R. Krpoun, Micromachined Electrospray Thrusters for Spacecraft Propulsion, PhD Thesis, Swiss Federal Institute of Technology Lausanne, Lausanne, Switzerland, 2009.

[21] M. A. Lieberman, A. J. Lichtenberg, Principles of plasma discharges and materials processing, 2nd Edition, Wiley-Interscience, Hoboken, NJ, 2005.

[22] B. Ferda, Retarding Potential Analyzer Theory and Design, Princeton University, 2015.

[23] S. Dandavino, et al., Microfabricated electrospray emitter arrays with integrated extractor and accelerator electrodes for the propulsion of small spacecraft, Journal of Micromechanics and Microengineering, Vol. 24, 2014.

[24] J. Perel, T. Bates, J. Mahoney, R.D. Moore, and A. Y. Yahiku, Research on a Charged Particle Bipolar Thrustor, AIAA Electric Propulsion and Plasmadynamics Conference September 11-13, Colorado Springs, CO, 1967.

[25] Massachusetts Institute of Technology, Electrospray thruster engineering, http://spl.mit.edu/ electrospray-thruster-engineering [Accessed June 2019]

[26] B. Q. T. Si, D. Byun, and S. Lee, Experimental and theoretical study of a cone-jet for an electrospray microthruster considering the interference effect in an array of nozzles, Journal of Aerosol Science, Vol. 38, pp. 924-934, 2007.

[27] Engineering ToolBox, Surface Tension, https://www.engineeringtoolbox.com/surface-tensiond_962.html [Accessed June 2019].

[28] ESI Group, CFD-ACE+, https://www.esi-group.com/software-solutions/virtual-environment/cfdmultiphysics/ace-suite/cfd-ace [Accessed July 2019].

[29] R. Wirz, Improved electrospray life and performance through coupled experiments and modeling, AFRL Electrospray / Propulsion in Space Workshop May 20-22, Los Angelos AFB, 2019.

[30] Physical Properties of Glycerine and Its Solutions, Glycerine Producers' Association, New York, 1963.

[31] M. Gamero-Costaño, and V. Hruby, Electrospray as a Source of Nanoparticles for Efficient Colloid Thrusters, Journal of Propulsion and Power, Vol. 17, 2001. 
[32] V. L. Piscane, The Space Environment and its Effects on Space Systems, AIAA, Reston, VA, 2008. 


\title{
APPENDICES
}

\author{
Appendix A \\ ADDITIONAL CFD VISUALIZATIONS
}

\section{Validation Case}

Mesh consists of 800 nodes and 703 cells. Left surface set to 0 V. Right surface set to 1000V. Separation distance in one meter. Simulation ran for 250 iterations.

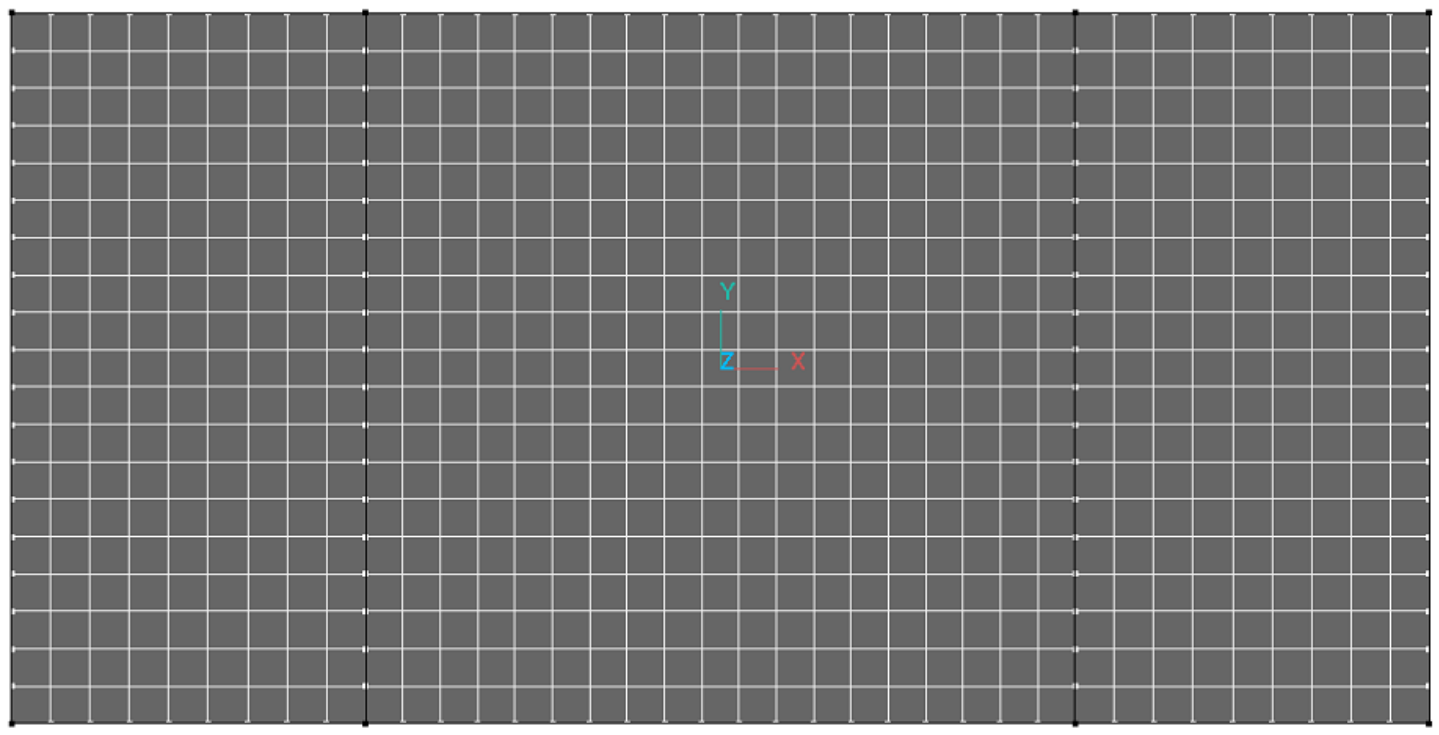

Figure 1: Mesh of the validation case, representing two flat surfaces separated by a distance.

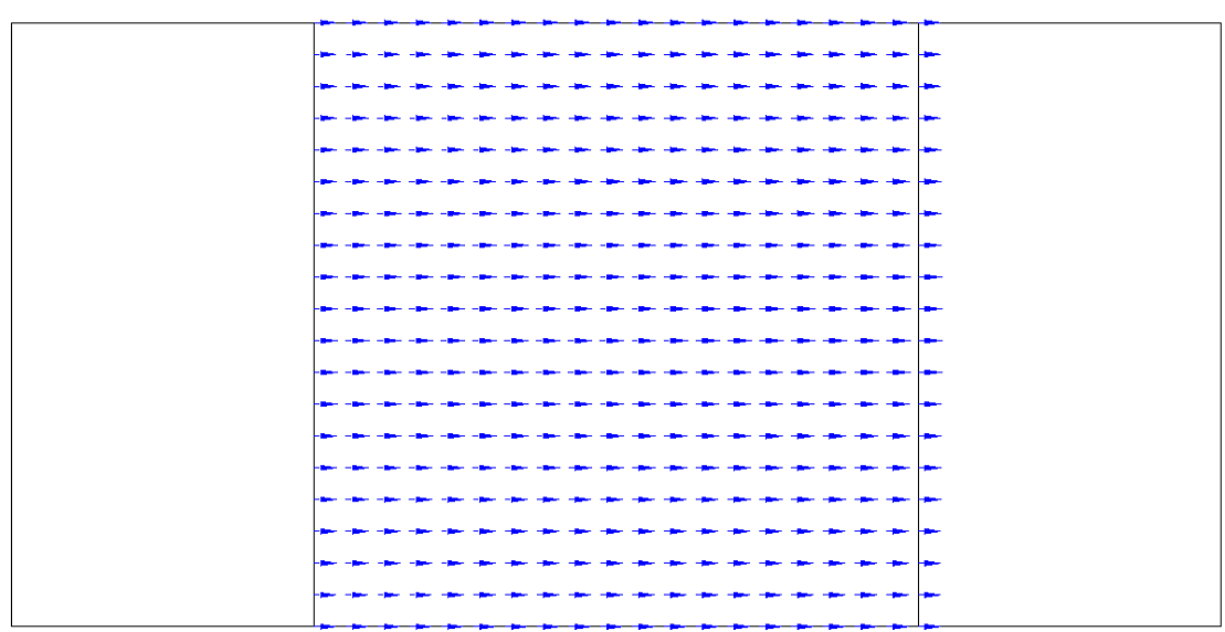

Electric Field - 1000 V/m

Figure 2: Resulting electric field vector field of the validation case. 


\section{Demonstration Thruster}

Mesh consists of 9470 nodes and 8890 cells. Main structure and emitter on left are set to $2700 \mathrm{~V}$. Extractor on right is set to $0 \mathrm{~V}$. Extractor distance is set to 0.1 inches. Simulation ran for 100 iterations.

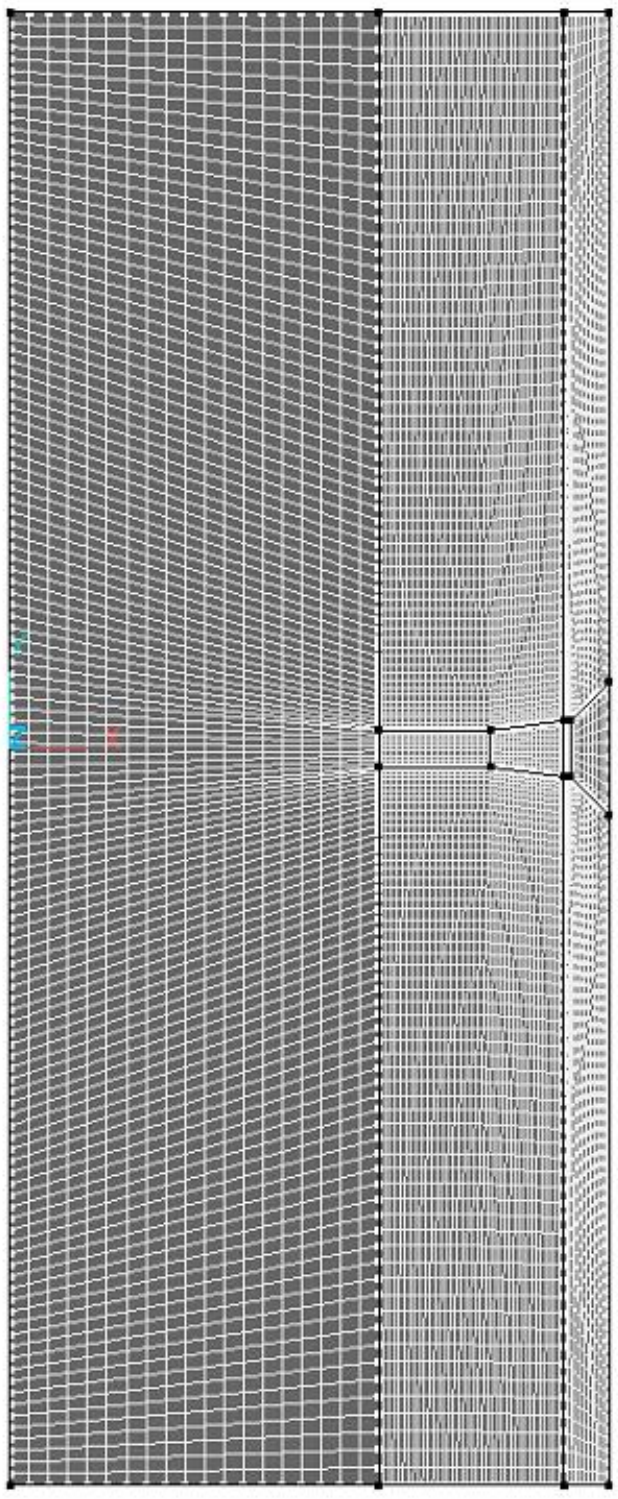

Figure 3: Mesh used for CFD analysis on the Demonstration Thruster. 


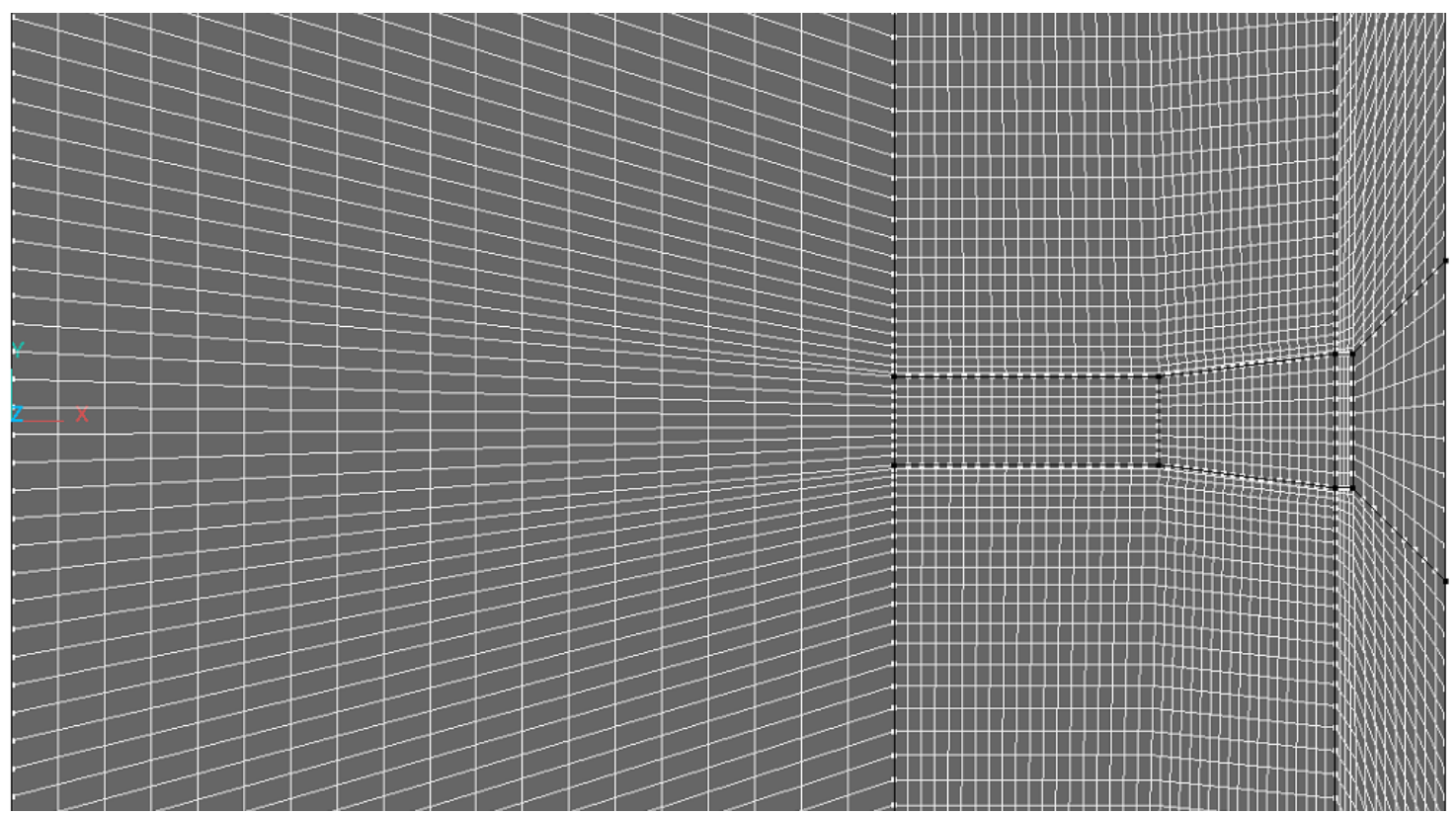

Figure 4: Close up on mesh near emitter used for CFD analysis on the Demonstration Thruster.

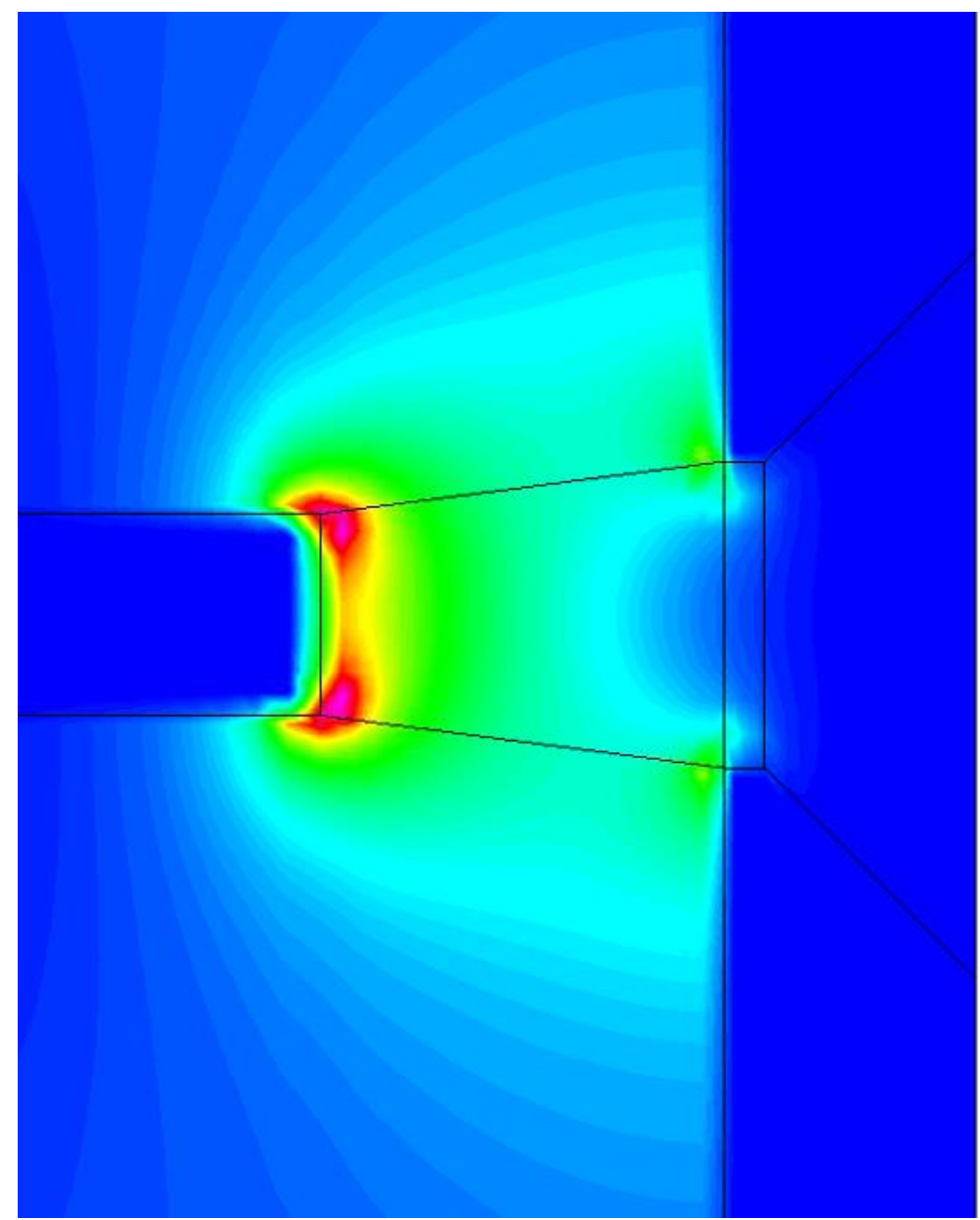

Electrostatic Energy - J

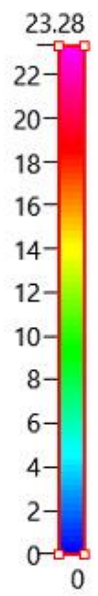

Figure 5: Electrostatic energy plot near the single emitter and extractor on the Demonstration thruster. 


\section{Research Thruster}

Mesh consists of 13234 nodes and 12064 cells. Main structure and emitter on left are set to $3700 \mathrm{~V}$. Extractor on right is set to $0 \mathrm{~V}$. Extractor distance is set to 0.07 inches. Simulation ran for 100 iterations.

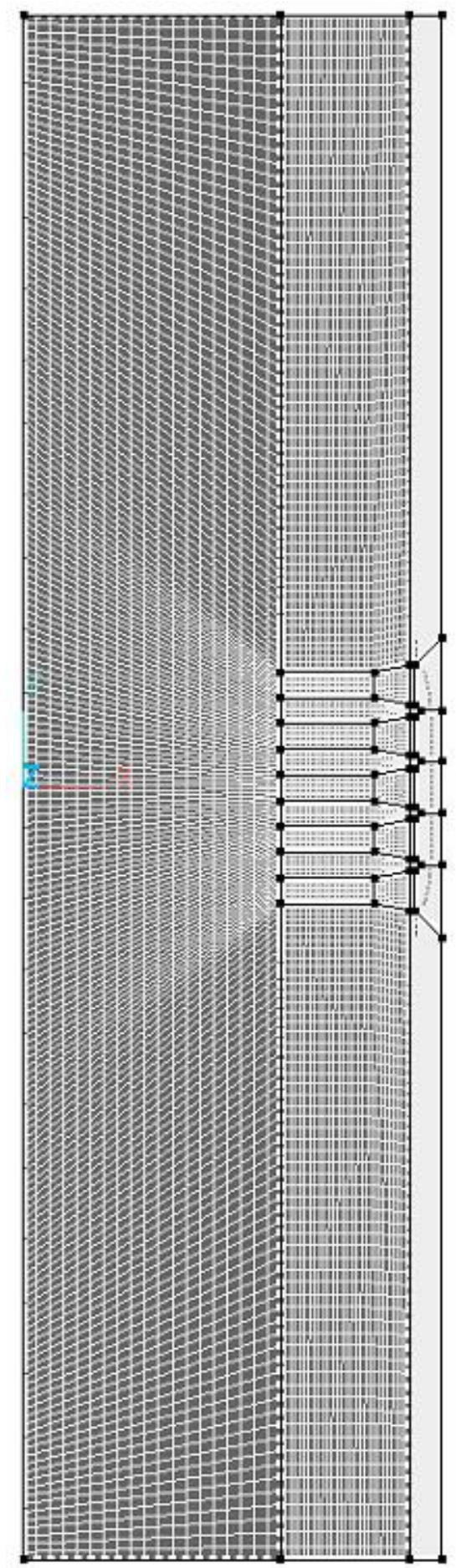

Figure 6: Mesh used for CFD analysis on the Research Thruster. 


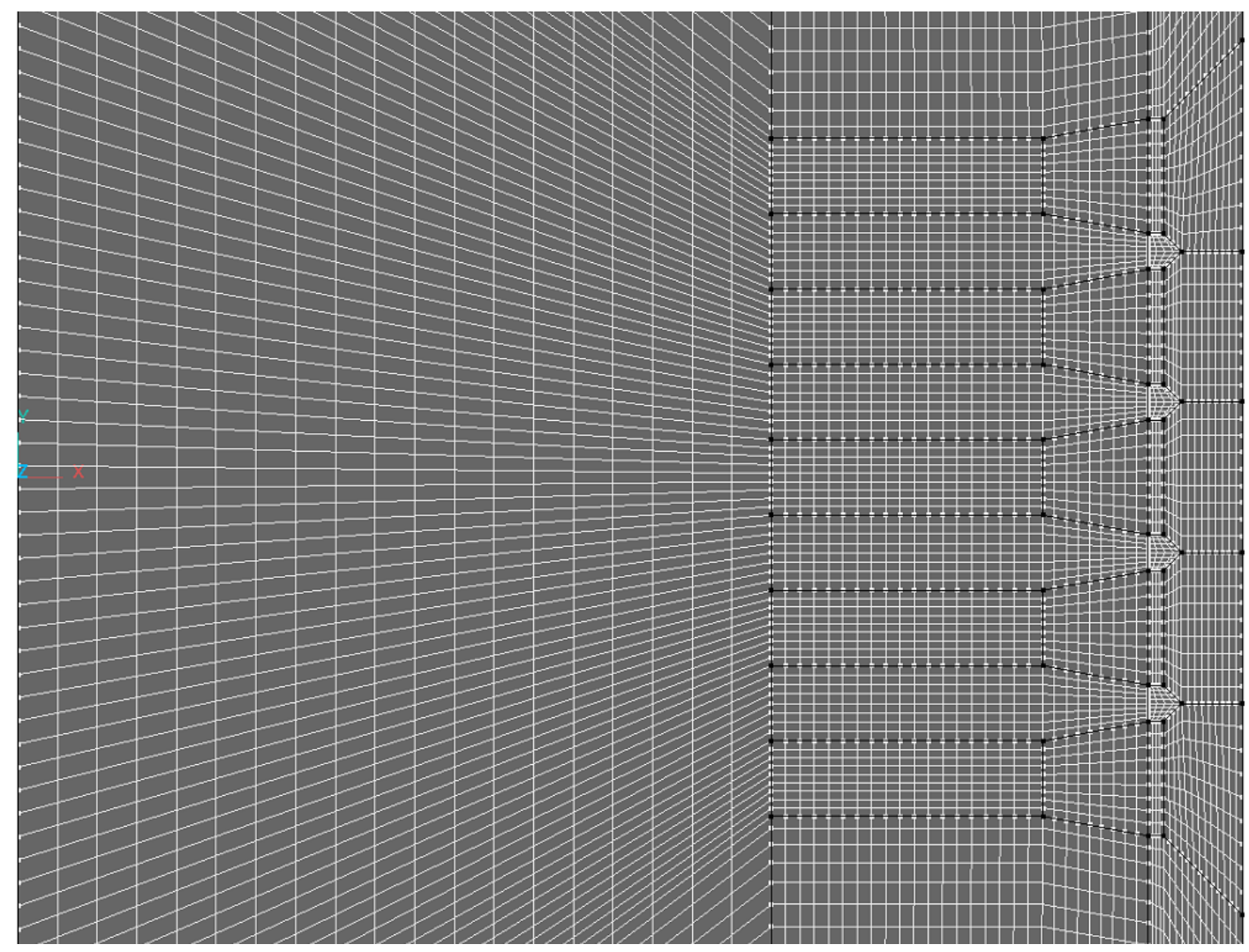

Figure 7: Close up on mesh near emitters used for CFD analysis on the Research Thruster. 


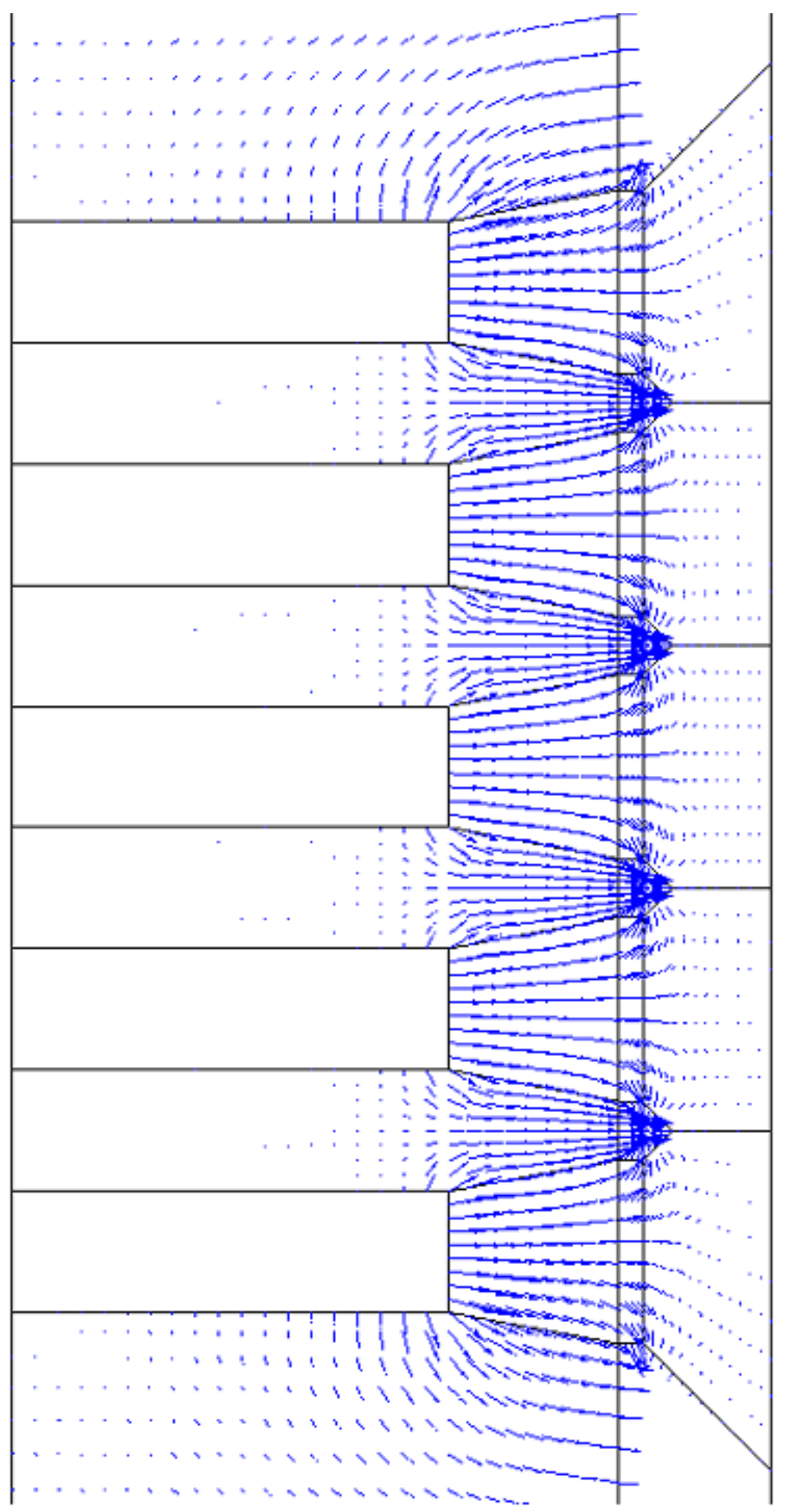

Figure 8: Electric field strength vector plot for all five emitters and extractor on the Research thruster. 


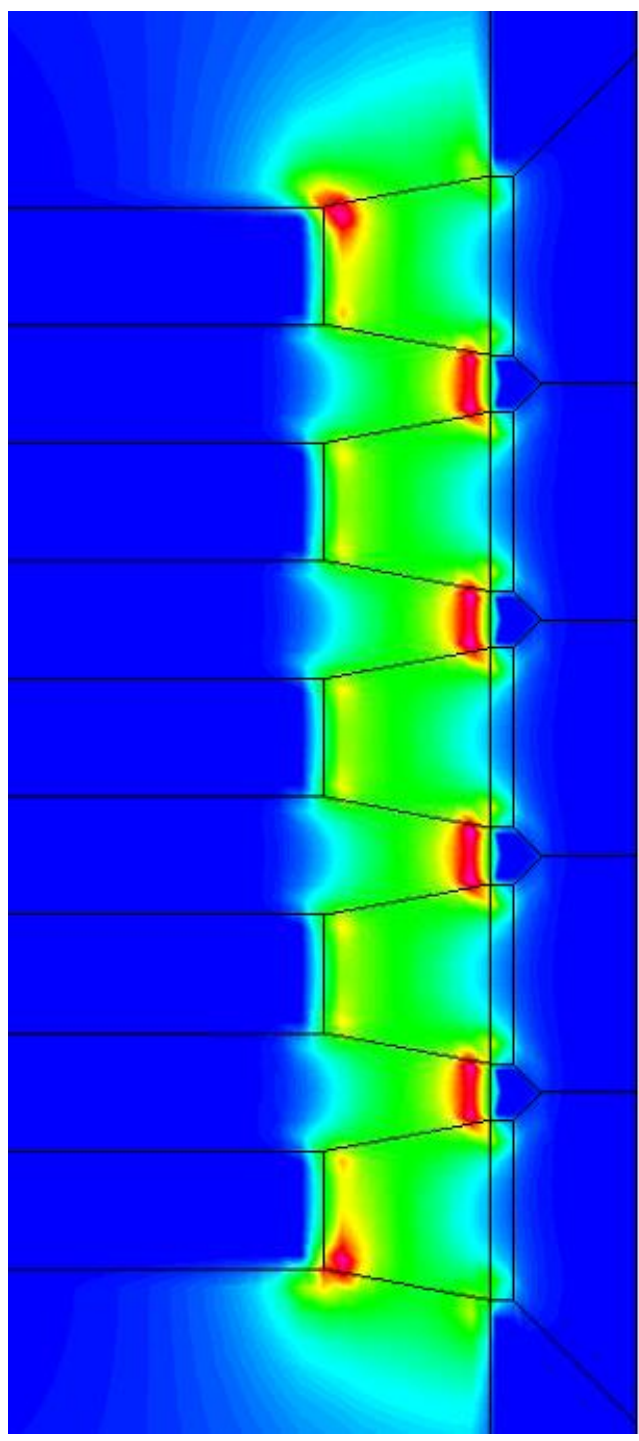

\section{Electrostatic Energy - J}

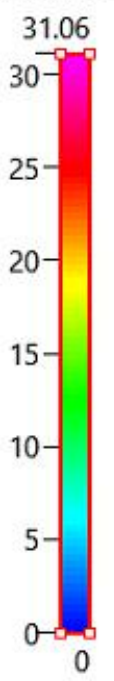

Figure 9: Electrostatic energy plot for all five emitters and extractor on the Research thruster. 
Appendix B

THRUSTER ASSEMBLY AND OPERATION GUIDE

\section{Demonstration Thruster}

\section{Assembly}

The Demonstration thruster can be assembled in full with the following tools:

- \#2 Phillips screwdriver

- 7/16" Wrench/socket wrench

All parts necessary for the main thruster assembly are shown in figure 1. All machine screws and nuts are in \#10-32 thread. All fasteners are stainless steel with the exception of the Nylon hardware meant to electrically isolate the extractor from the emitter and structure.

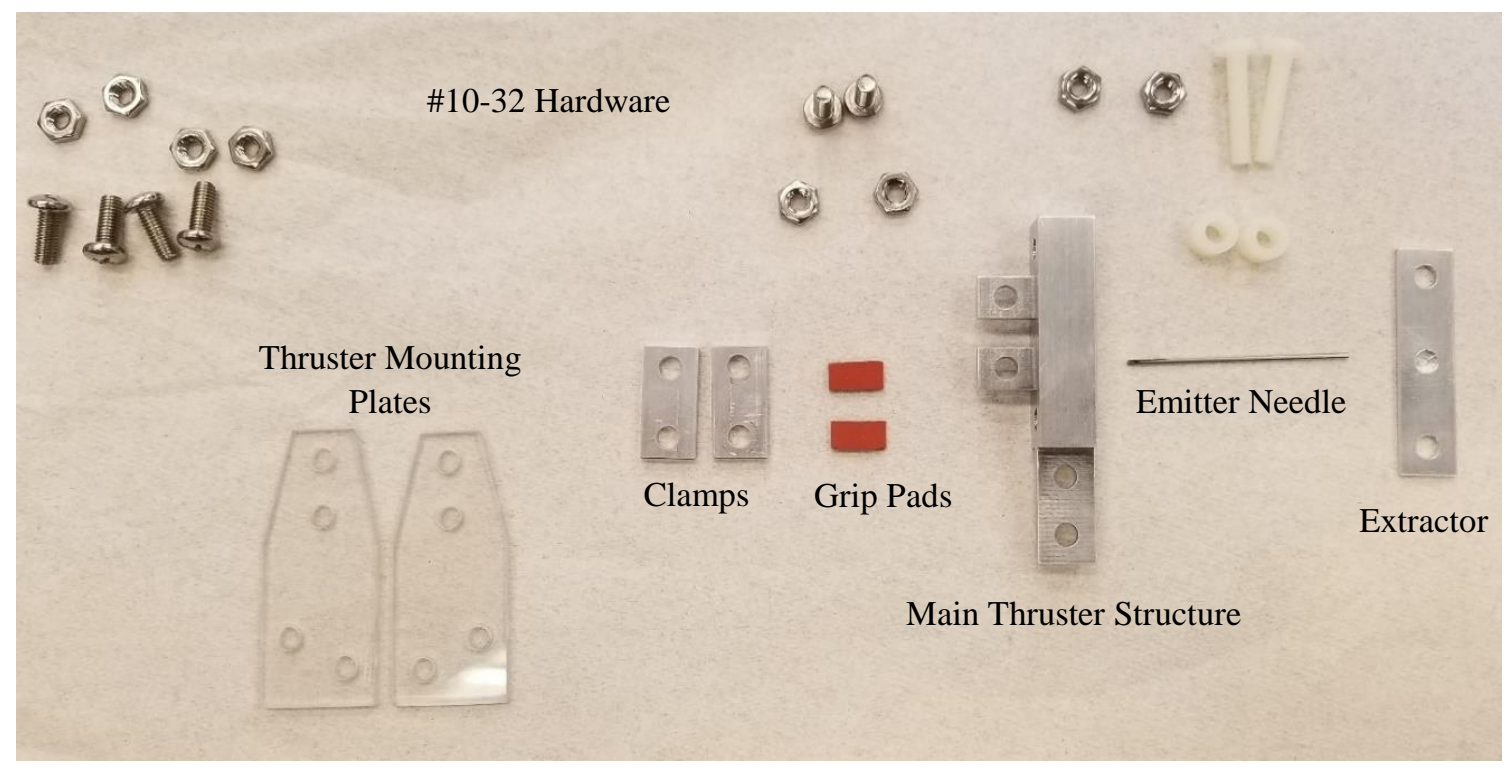

Figure 1: All parts for the Demonstration thruster laid out for assembly.

Assembly starts with the primary thruster components. An exploded CAD assembly model can be seen in figure 2 .

1. Insert the emitter needle through the hole in the main thruster structure. Have the tip of the emitter extend just past the structure face for now.

2. Loosely secure the clamp and the grip pads on the emitter to fix the emitter in its position temporarily. Use $1 / 2$ " length stainless screws and a stainless nut.

3. Use the nylon hardware and stainless nuts to attach the extractor to the main thruster structure. The spacers are in-between the parts and the screw heads are on the extractor side.

4. At this point the distance between the extractor and emitter can be set using the precision spacers shown in figure 3 . There are five sizes provided; from 0.060 " to 0.100 " in 0.010 " intervals. Start by holding the spacer of the inside face of the extractor and then moving the emitter needle forward until it is flush with the spacers. Rotate the emitter in place to remove any distortion in the grip pads that will act like a spring and move the emitter after its let go. Firmly tighten the clamps until they are in full contact with the tabs on the main thruster structure. 
5. Attach the main thruster structure to the polycarbonate plates using $1 / 2$ " length stainless screws and stainless nuts.

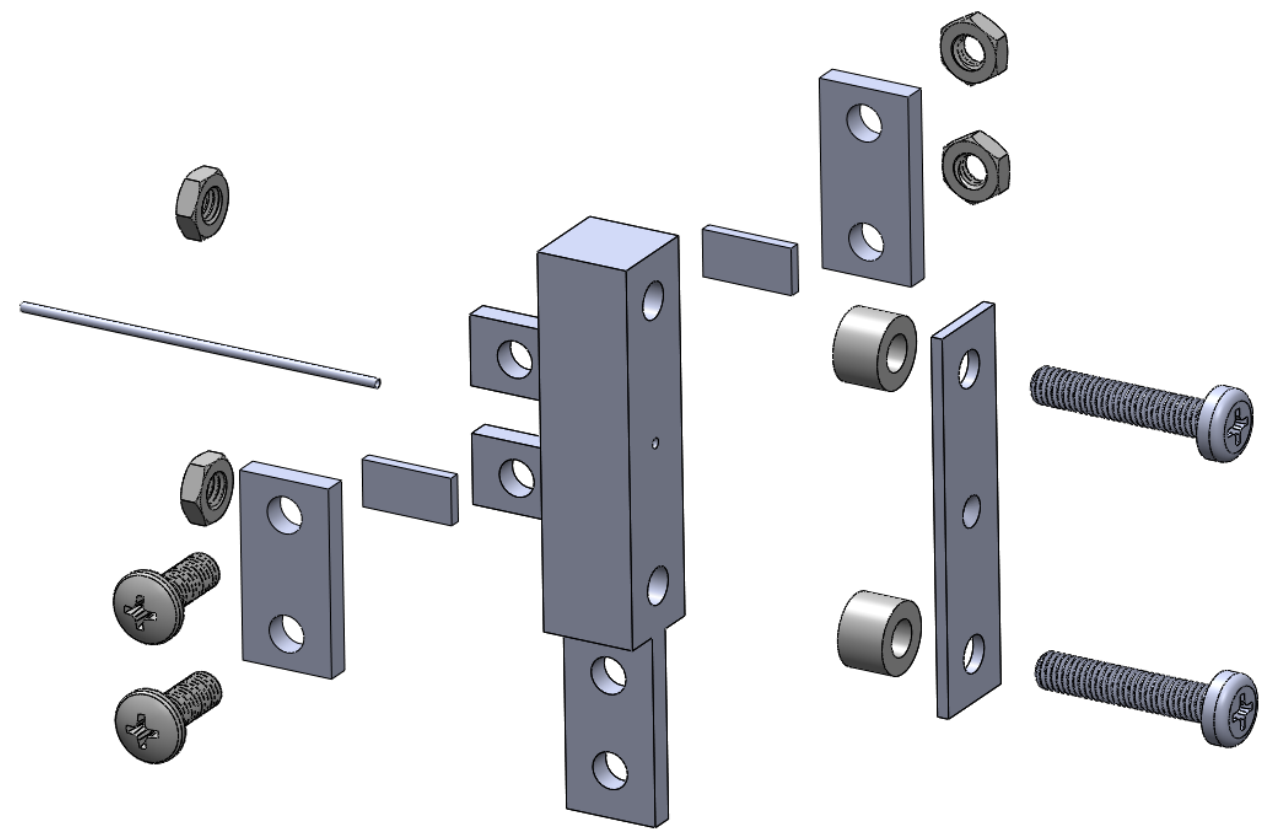

Figure 2: Exploded view of the Demonstration thruster assembly.

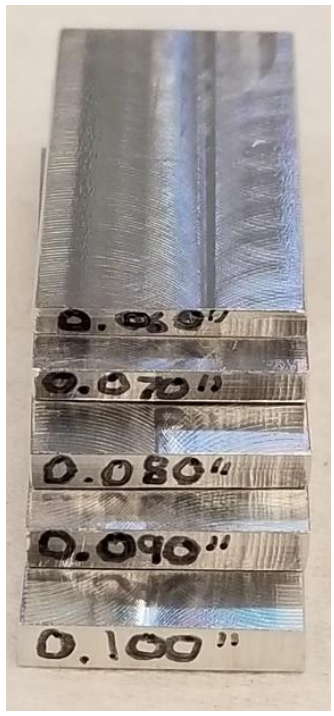

(a)

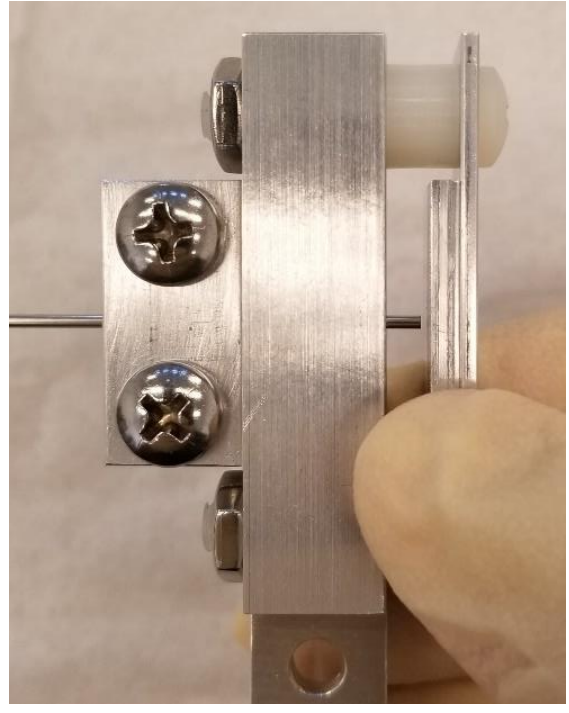

(b)

Figure 3: Precision spacers (a) and distance setting procedure example (b).

To set the thruster up in a testing capacity, as can be seen in figure 4, follow this procedure. The wires described here are all 22 AWG HV wire insulated with red silicone. Ring terminals are all tin coated copper and sized to work with \#10 screws.

1. Attach the wire leads to the extractor and the main thruster structure. The HV lead for the main thruster structure is 65 " long and has ring terminals on both sides. Attach this lead onto the face furthest from the extractor using the top nylon screw and stainless nut as the anchor. The lead to the 
extractor is 14" long and has a ring terminal on one end and a male BNC connector on the other end. Attach the extractor lead on the outside face of the extractor using the top nylon screw as the anchor. A close up of this step is shown in figure 5.

2. At this point, the axes of the emitter and the extractor hole can be aligned. Do this by looking down the bore of the emitter and aligning it by eye to the hole in the extractor. The extractor will be able to move in two directions to align well enough. Tighten the nylon screws when aligned. An example of this is shown in figure 6.

3. Attach the polycarbonate mounting plates to the L-bracket on the plywood base using $3 / 4$ " stainless crews and nuts.

4. Attach the collection plate to its L-bracket on the plywood base using nylon screws and stainless nuts. The collection plate is separated from the L-bracket using silicone strips. A close up of this can be seen in figure 7 .

5. Attach the PTFE plate to the back side of the thruster using Kapton tape. Try to have this extend past the extractor as little as possible.

6. Connect the wire lead to the collector plate using a 1/2" stainless screw and nut. This lead is 13 " long and has a ring terminal on one end and a male BNC connector on the other end.

7. If necessary, assemble the polycarbonate box using 1/4" long \#8-32 screws and brackets. This is not attached to the plywood base in any way.

8. The syringe pump is placed on the end of the plywood base away from the thruster assembly. The syringe needle is connected to the emitter using a 9" length of $1 \mathrm{~mm}$ ID silicone tube.

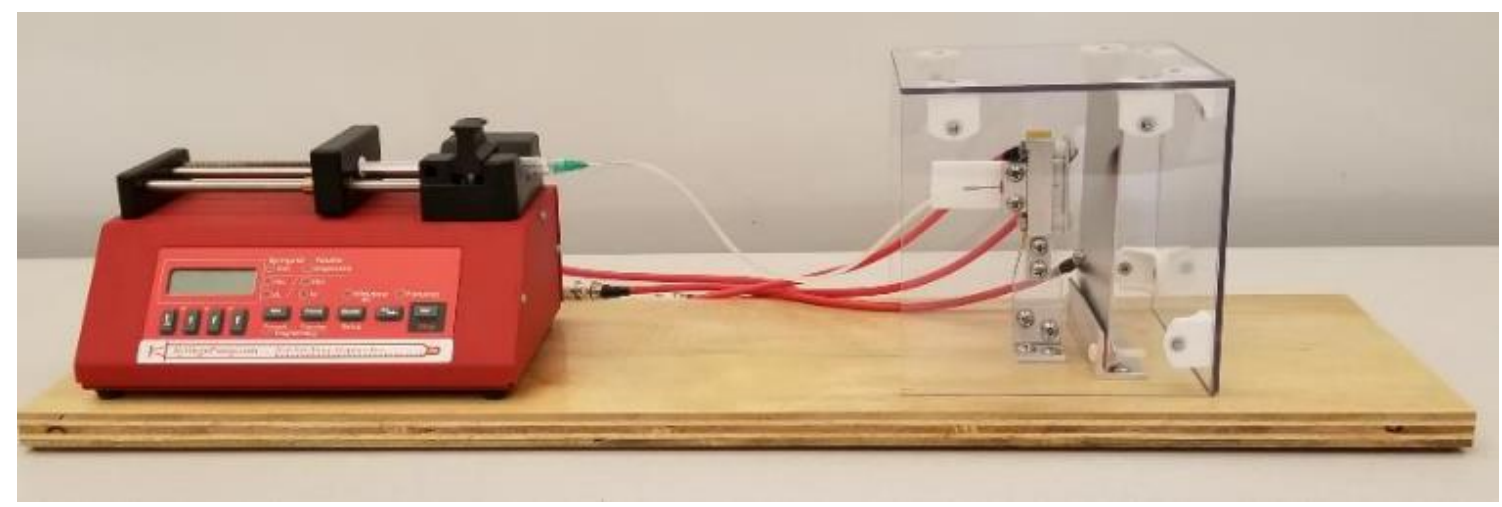

Figure 4: Full assembly of Demonstration thruster test setup.

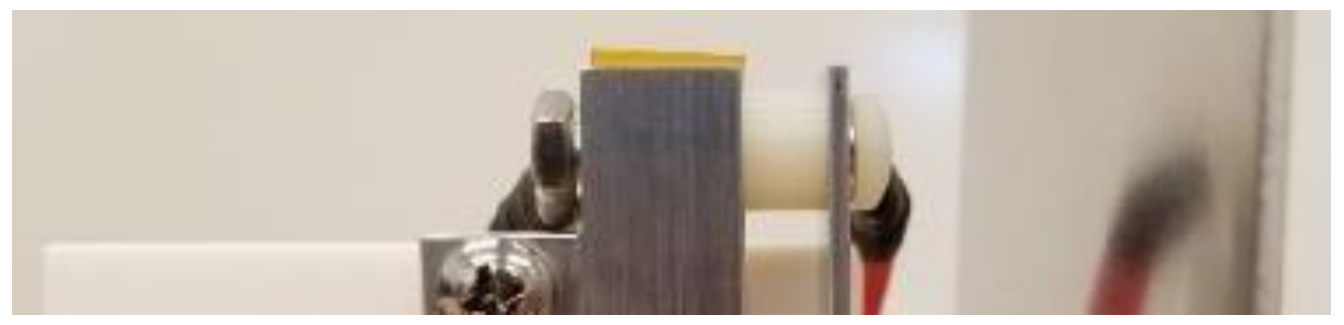

Figure 5: Close up of thruster lead connections. 


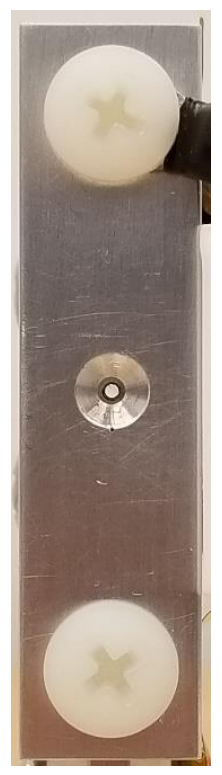

Figure 6: Close up emitter and extractor alignment.

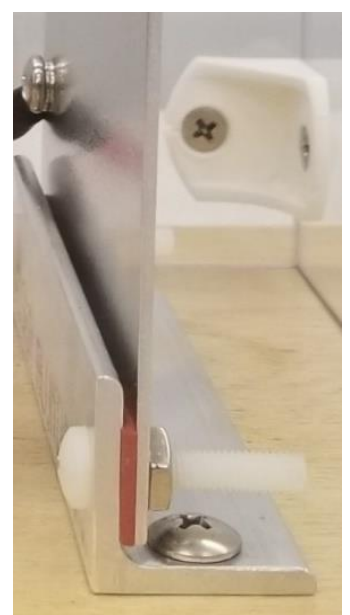

Figure 7: Close up of collector plate fasteners.

\section{Diagnostic Equipment Connections}

Attachments to the diagnostics equipment are as follows. A simplified electrical diagram is given in figure 8.

1. High voltage is delivered to the thruster using a series of cables and wires. Starting a $10 \mathrm{ft}$ RG-8U cable with a custom input on one end and a male PL-2590 connection on the other. A picture of the custom end is given in figure 9(a). This connects to a male to male PL-259 adaptor and then a 24" RG-8U cable with male PL-259 Cables on both ends, where one end of this cable is soldered to a 22 AWG HV wire that leads to a ring terminal. This special cable is shown in figure 9 (b). This ring terminal is connected to the wire leading to the main thruster structure using a $1 / 2$ " stainless screw and nut.

2. Also from the high voltage connection is a lead to the HV probe for the oscilloscope. This lead is a 15 " 22 AWG HV wire with ring terminals on both ends. One terminal is connected to the same junction as in the previous step and the other in on the probe. The three-way junction is shown in 
figure 10. The probe has a BNC cable that connects to the oscilloscope and a ground wire that is connected to ground with an alligator clip.

3. Connections for the pico-ammeter were made using BNC cables connected to the male BNC connectors on the wires connected to the thruster components. The BNC cables used also have male connectors on both sides, so male-to-male BNC adaptors were used. To facilitate easier switching between measuring currents on the extractor and collector surfaces, a shorter BNC cable was connected to the pico-ammeter and was connected to either of the two BNC cables leading to the thruster components. This removes the need to switch out the cables at the back of the equipment rack.

4. Ground connections for the power supply, pico-ammeter, HV probe, and BNC cables are all consolidated to a single mounting point of the equipment rack to avoid ground loop interferences, as seen in figure 11 .

5. Data connections to a computer from the pico-ammeter and oscilloscope are necessary for data collection. The pico-ammeter communicates through RS-232 DB 9 output, so an adaptor going from DB 9 to USB A 2.0 was used to connect with the computer. The oscilloscope communicates through USB B 2.0, so a USB B 2.0 to USB A 2.0 cable was used to connect with the computer.

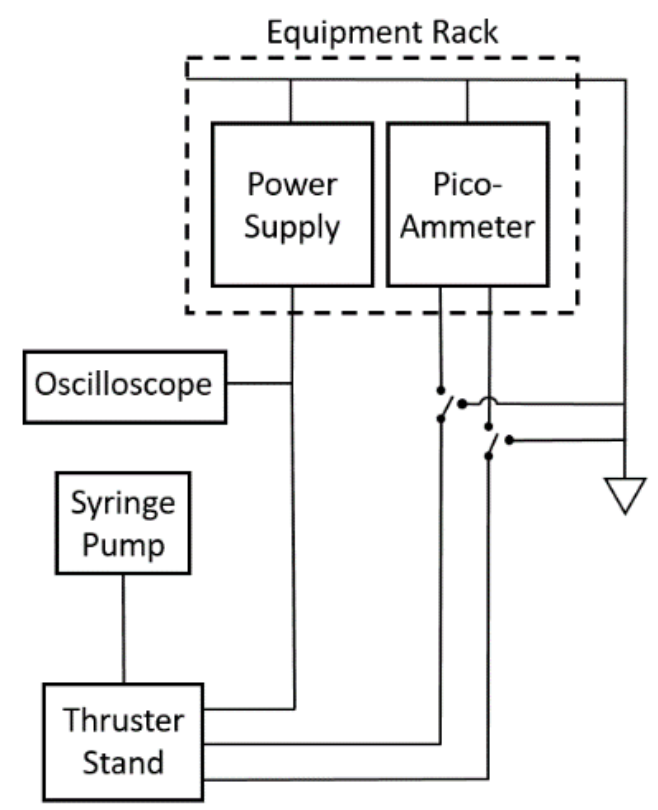

Figure 8: Electrical diagram of the Demonstration thruster setup 


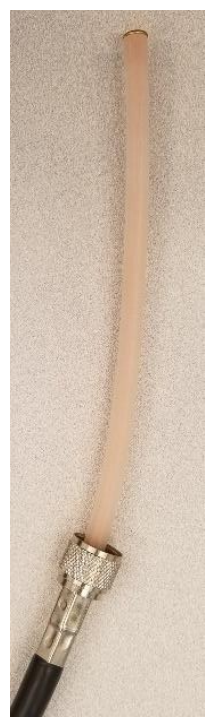

(a)

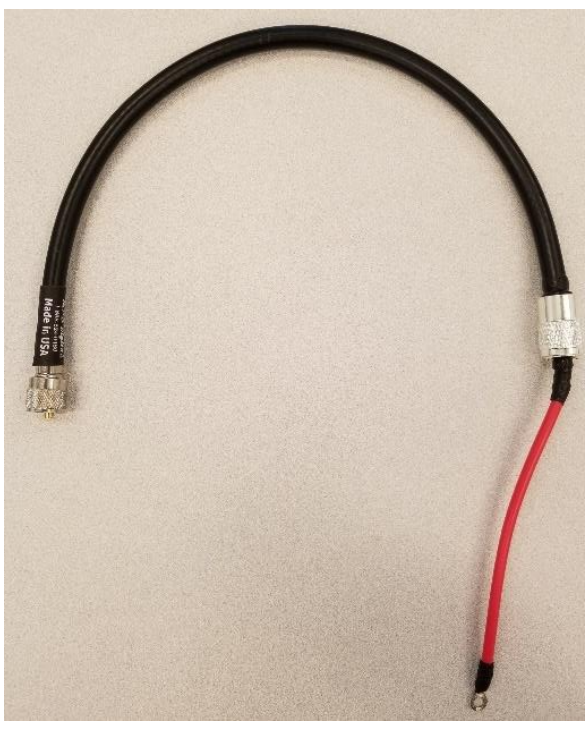

(b)

Figure 9: Custom input to power supply (a) and the RG-8U cable to HV Wire component (b).

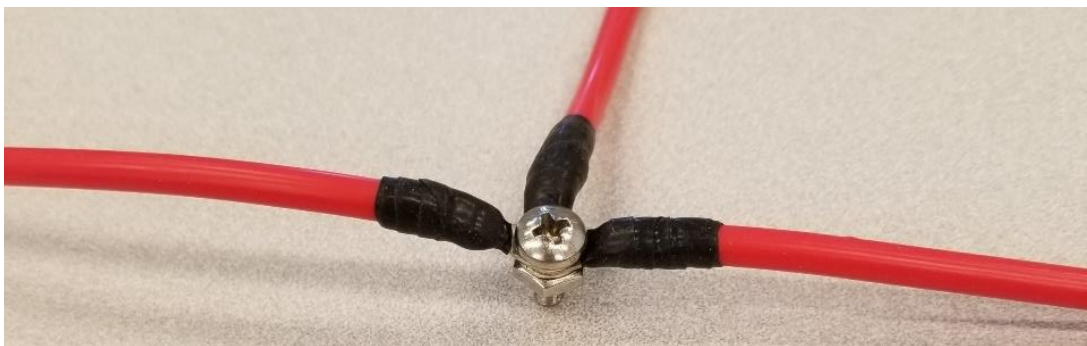

Figure 10: Three-way high voltage junction.

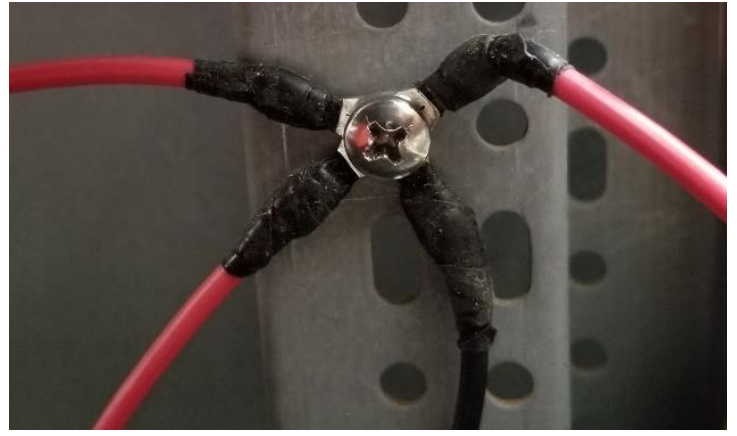

Figure 11: Ground connections for diagnostic equipment on equipment rack.

\section{Research Thruster}

\section{Assembly}

The Research Thruster can be fully assembled with the following tools:

- \#2 Phillips screwdriver

- $5 / 16$ "Wrench/socket wrench 
- 7/16" Wrench/socket wrench

- $1 / 16 "$ Hex Allen wrench

- 3/16" Hex Allen wrench

- $1 / 4$ " Hex Allen wrench

All parts required for the main thruster assembly are shown in figure 12. All fasteners are in \#10-32 thread with the exception of the \#6-32 set screws for the emitter block. All fasteners are stainless steel with the exception of the PTFE spacers and machine screws meant to electrically isolate the extractor.

Assembly of the full testing setup begins with the thruster assembly itself. An exploded CAD assembly model can be seen in figure 13 .

1. Secure the emitter block to the main thruster structure by tightening the set screws against the tabs of the emitter block. After this point, the assembly is very similar to the Demonstration thruster.

2. Insert the five emitters into the holes through the emitter block so they are just past the outer face of the emitter block.

3. Loosely secure the emitters in place by tightening the clamps and grip pads over the emitters using the $1 / 2$ " machine screws and nuts.

4. Use the PTFE hardware and stainless steel nuts to attach the extractor the main thruster structure, with the spacer in-between the two components and the head of the screw on the outside face of the extractor.

5. At this point the distance between the extractor and emitter can be set using the precision spacers shown in figure 3 (a). There are five sizes provided; from 0.060 " to 0.100 " in 0.010 " intervals. Start by holding the spacer of the inside face of the extractor and then moving the emitter needle forward until it is flush with the spacers. Rotate the emitters in place to remove any distortion in the grip pads that will act like a spring and move the emitter after its let go. Firmly tighten the clamps until they are in full contact with the tabs on the main thruster structure. An example of this process is shown in figure 14.

6. Attach the main thruster structure to the PTFE mounting plates using 3/4" length stainless screws and stainless nuts. 


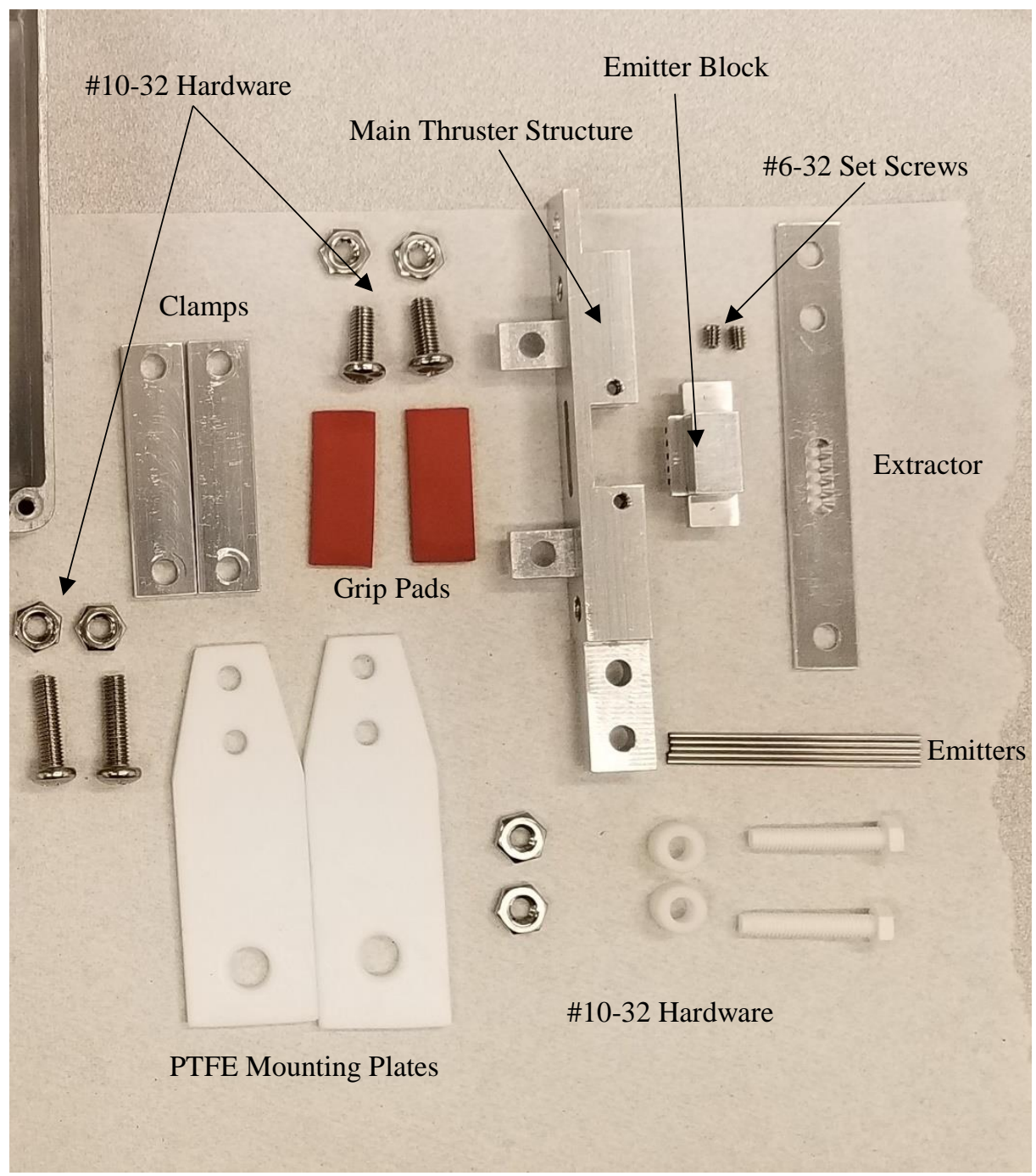

Figure 12: All parts of Research thruster laid out prior to assembly. 


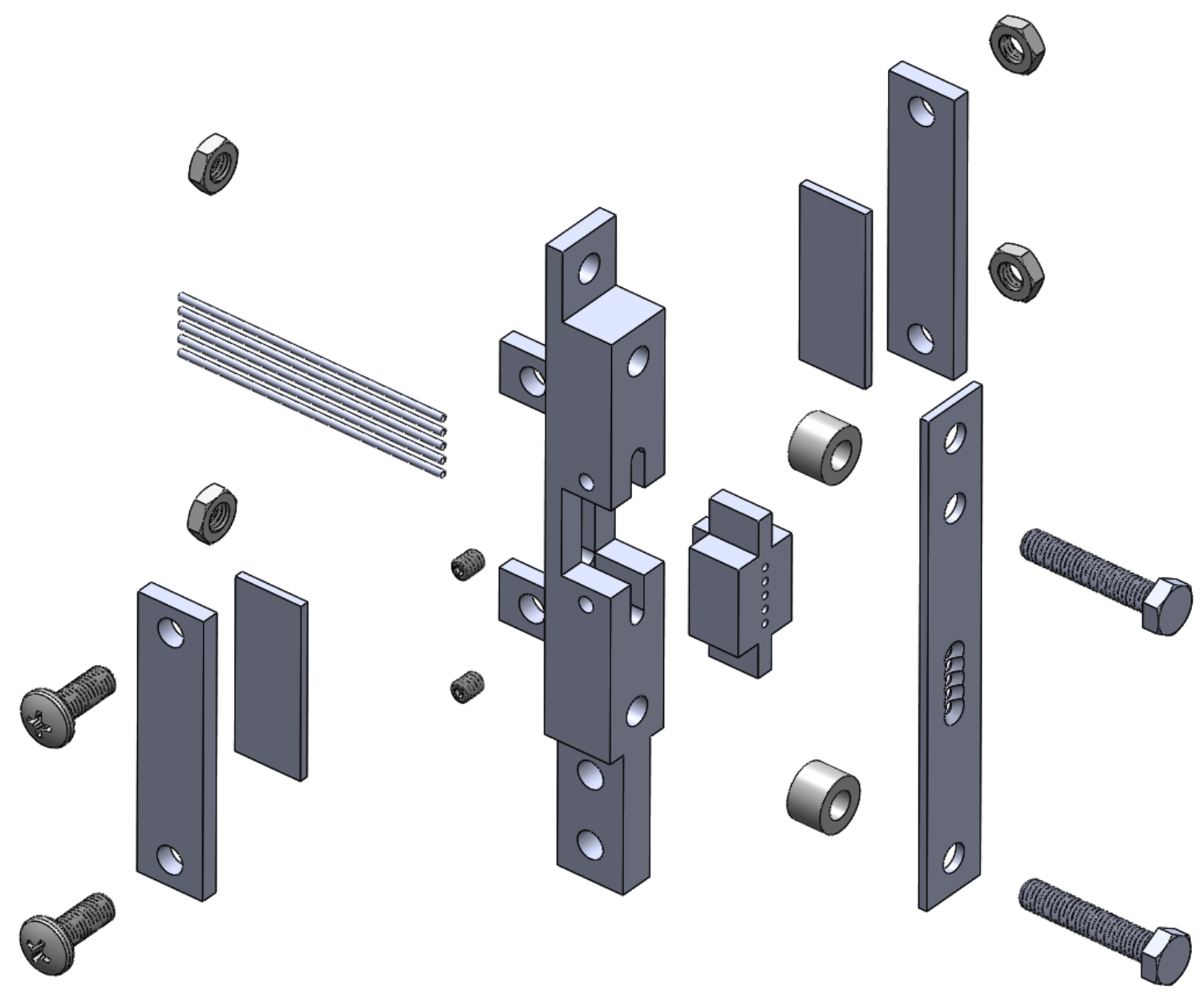

Figure 13: Exploded 3D CAD model of the Research thruster assembly.

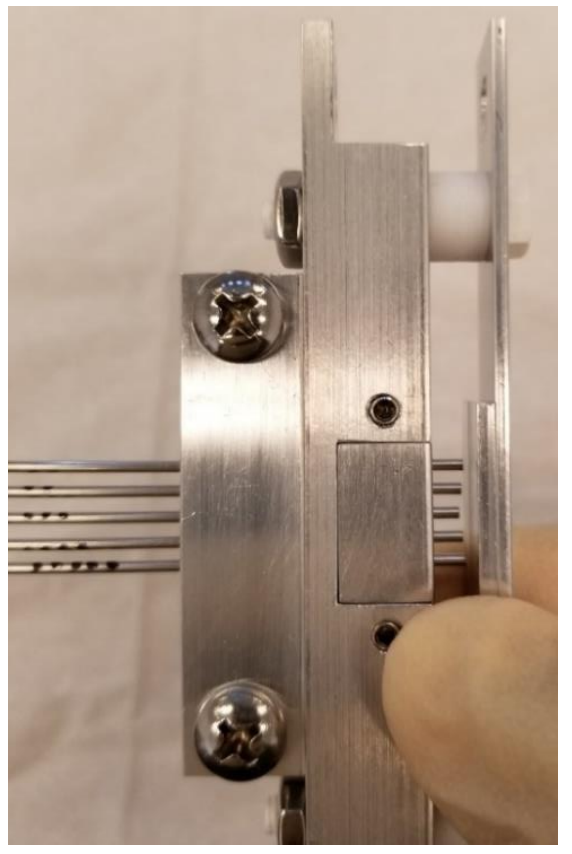

Figure 14: Extractor separation setting process example. 
The procedure to set the Research thruster up in a testing capacity, as can be seen in figure 15, is as follows. The wires described here are all 22 AWG HV wire insulated with red silicone unless otherwise specified. Ring terminals are all tin coated copper and sized to work with \#10 screws.

1. Assemble the emission containment box as shown in figure 15(a). An exploded view of this assembly is given in figure 15(b). The collector plate is separated from the outer box by silicone strips and PTFE screws. The wire leading to the collector plate is 37 " long and has a ring terminal on one end and a male BNC connector on the other.

2. Assemble the propellant line holder as shown in figure 16 . The two plates are simply attached using Kapton tape.

3. Assemble the test support structure apparatus as shown in figure 17. This structure is made using 1.5 " T-slotted single rails and various brackets. All fasteners for this assembly are in 5/16"-18 thread and stainless steel.

4. Attach wires to thruster components as shown in figure 18. The wire leading to the main thruster structure is 36 " long and has a ring terminal one end and a copper alligator clip on the other. The wire leading to the extractor is 37 " long and has a ring terminal on one end and a male BNC connector on the other.

5. With the wires attached to the thruster components, now is the best time to adjust the alignment of the emitter and extractor hole axes. An example of this alignment is shown in figure 19.

6. Mount all three previous assemblies onto the test support structure in a position that is aligned with the chamber viewport when placed inside the vacuum chamber.

7. For single emitter testing, assemble the propellant line as shown in figure 20 . The assembly is made from short sections of tube and a plastic 1/16" tubing tee. Flow is stopped at one end of the tees using a $1 / 16$ " diameter metal rod. Additional flow resistance for the propellant was added in the form of a Kimwipe wad, formed from tightly rolling a $1 / 4 \times 13 / 16$ " strip of the fabric around its shorter axis. The wad was inserted into the silicone tubing using narrow tweezers. Line to emitter is 1.5 ". Line from feed through is 23 ".

8. The propellant distribution assembly used for multi-emitter testing is shown in figure 21 . This assembly uses five plastic $1 / 16$ " tubing tees and short connecting pieces of silicone tubing of about $3 / 8$ " to allow propellant to flow into the five emitters. The silicone tubes leading to the emitters from the tees are 2.75" long. Again, flow is stopped at one end of the tees using a 1/16" diameter metal rod to seal against an arbitrary length of tubing. Additional flow resistance for the propellant was added in the same form of a Kimwipe wad as before, formed from tightly rolling a 1/4 x 13/16" strip of the fabric around its shorter axis. The wad was inserted into each of the silicone tubes using narrow tweezers.

9. The outer propellant distribution system, which directs flow to either the bleed line or to the thruster, is assembled as shown in figure 22. The tubing that leads creates the bleed line is 13 " long, and leads to the front most fluid feedthrough. The tubing that leads to the thruster is 5.5 " long and leads to the rearmost fluid feedthrough. The short section of tubing connecting to the syringe needle is 3 " long.

10. On the inside of the chamber the bleed line is fed into a glass beaker using a 5 " long section of tubing, as shown in figure 23. A piece of aluminum foil is used to prevent splatter from exiting the beaker and contaminating the chamber.

11. Secure the glass syringe with additional silicone grip pads in the syringe pump clamp as shown in figure 24, where the pad to the top of the picture goes in between the top of the syringe and the spring loaded clamp head. Without these pads, the syringe barrel will slip due to the vacuum pressure.

12. A picture of the total in-chamber assembly is given in figure 25 . 


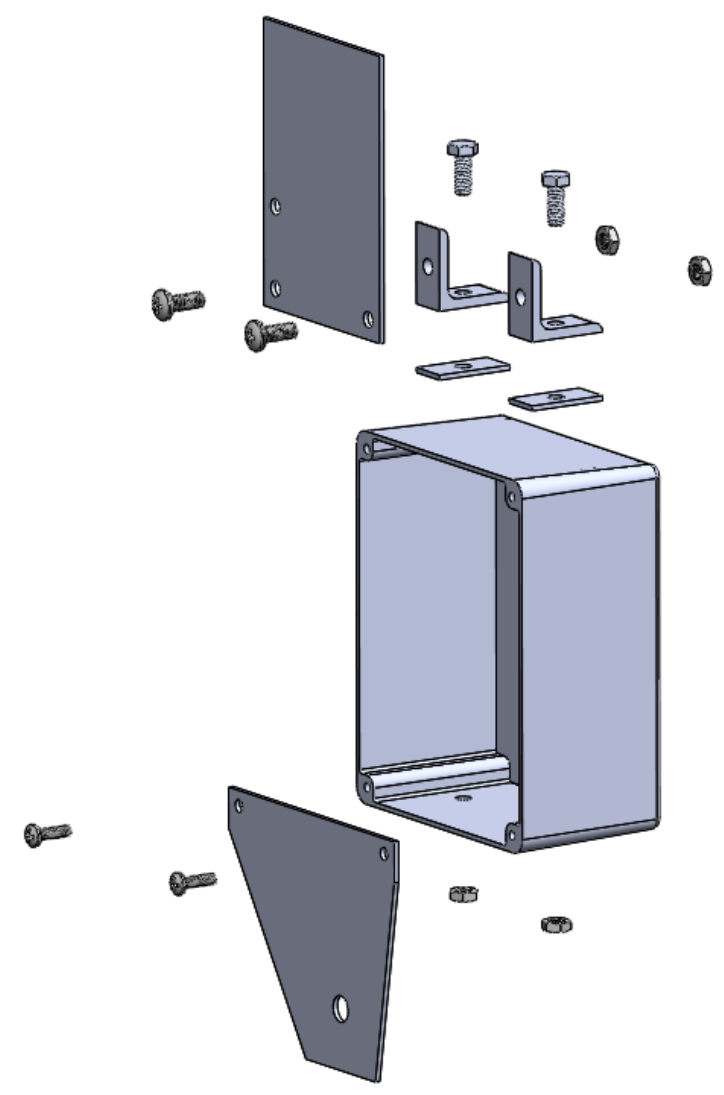

Figure 15: Exploded view of emission containment system.

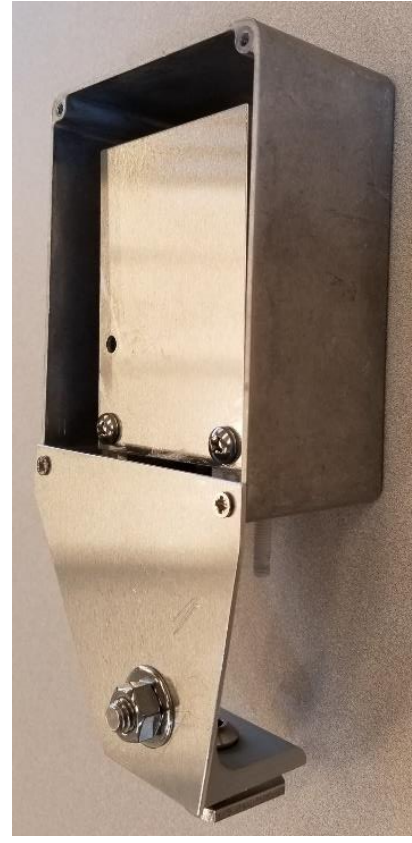

(a)

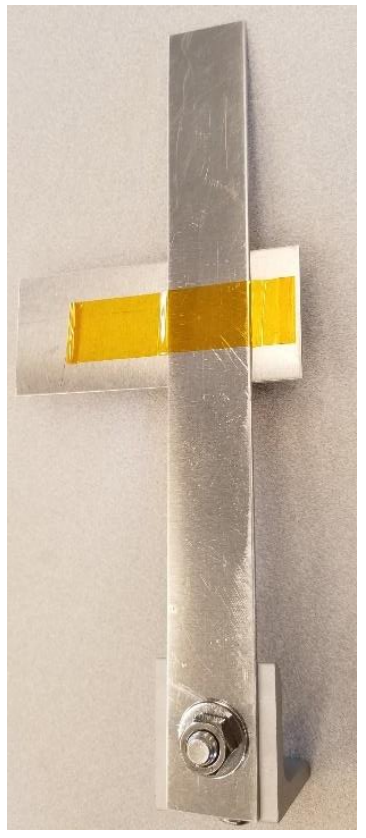

(b)

Figure 16: Assembled splash box (a) and propellant line holder (b). 


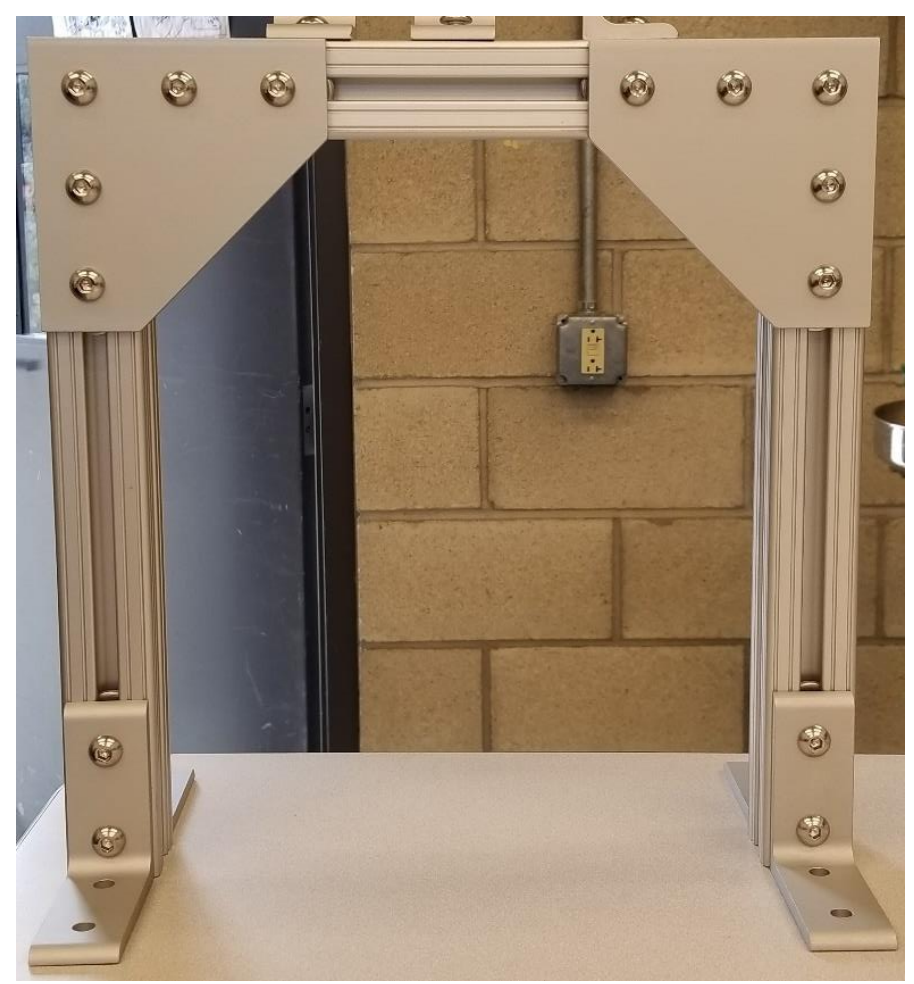

Figure 17: Chamber test support structure apparatus.

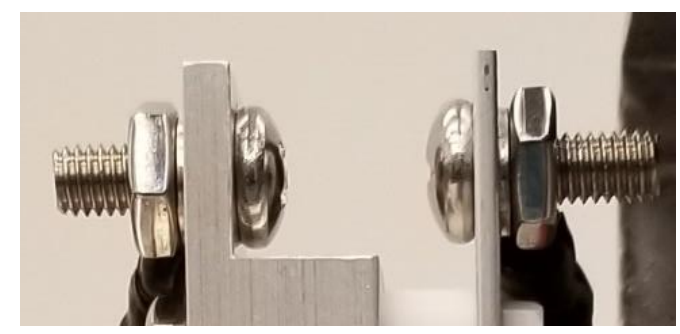

Figure 18: Close up of wire connections to thruster

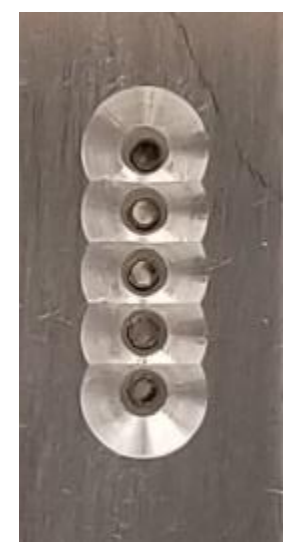

Figure 19: Research thruster emitter axis alignment example. 


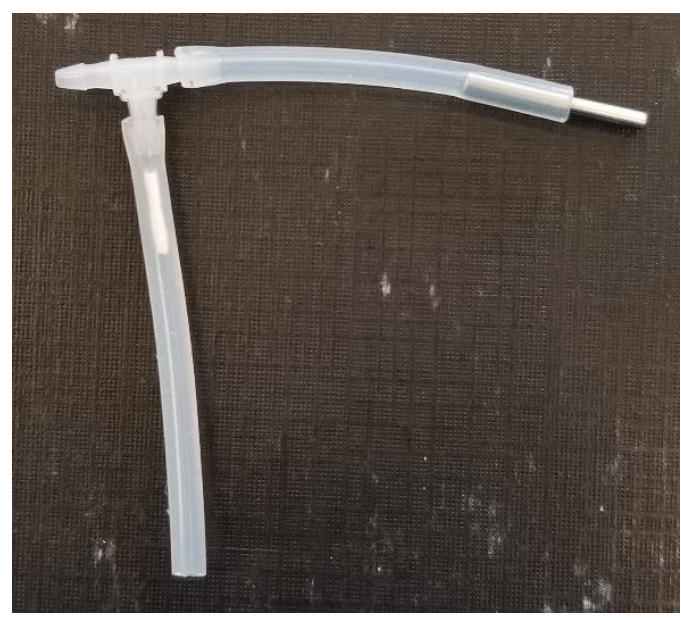

Figure 20: Single emitter prop tee assembly.

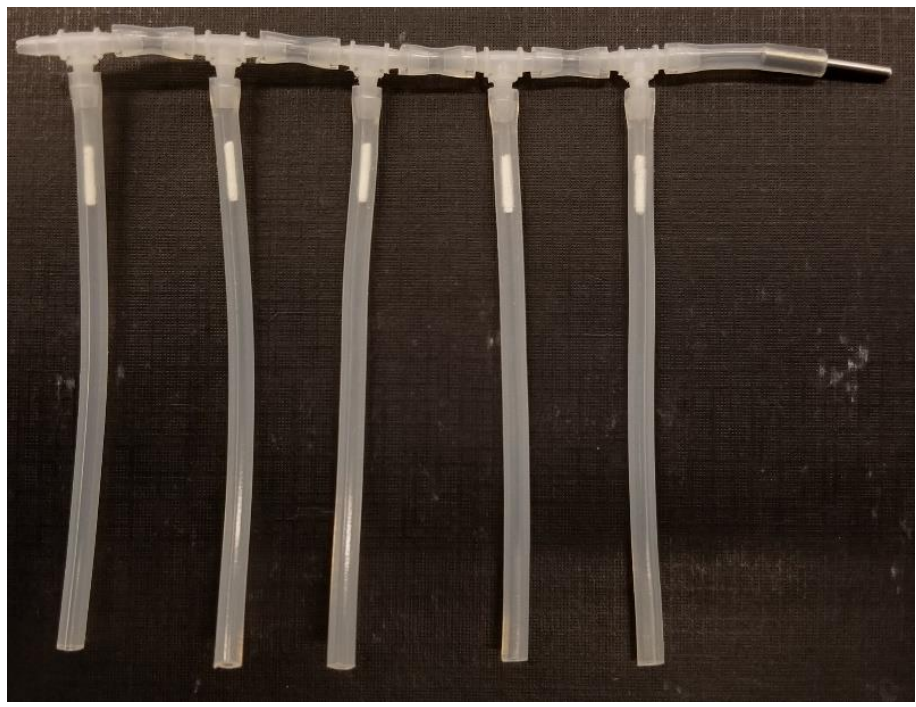

Figure 21: Multi-emitter propellant distribution assembly.

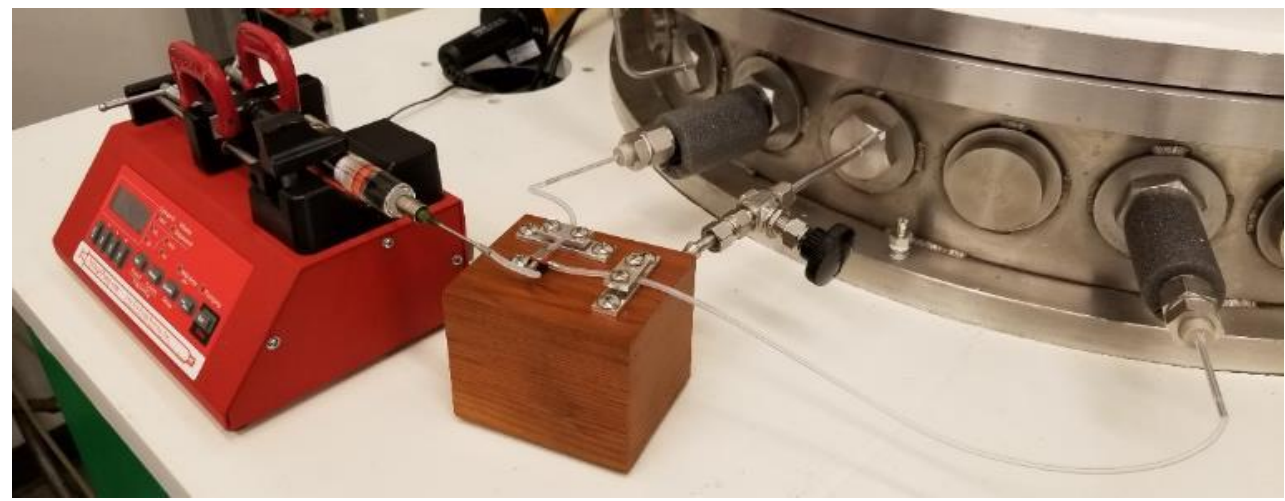

Figure 22: Propellant distribution system on the outside of the chamber. 


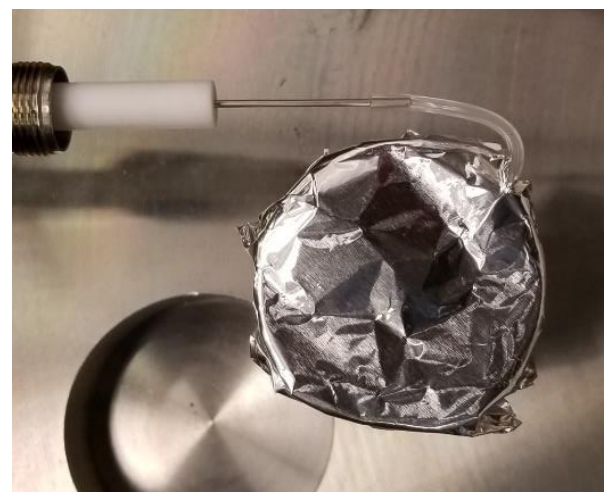

Figure 23: Bleed line path to glass beaker on the inside of the chamber.

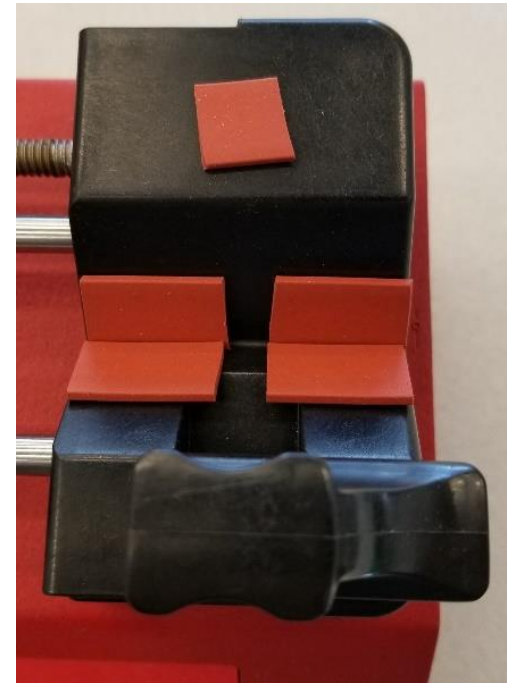

Figure 24: Grip pads used to secure glass syringe in syringe pump clamp. 


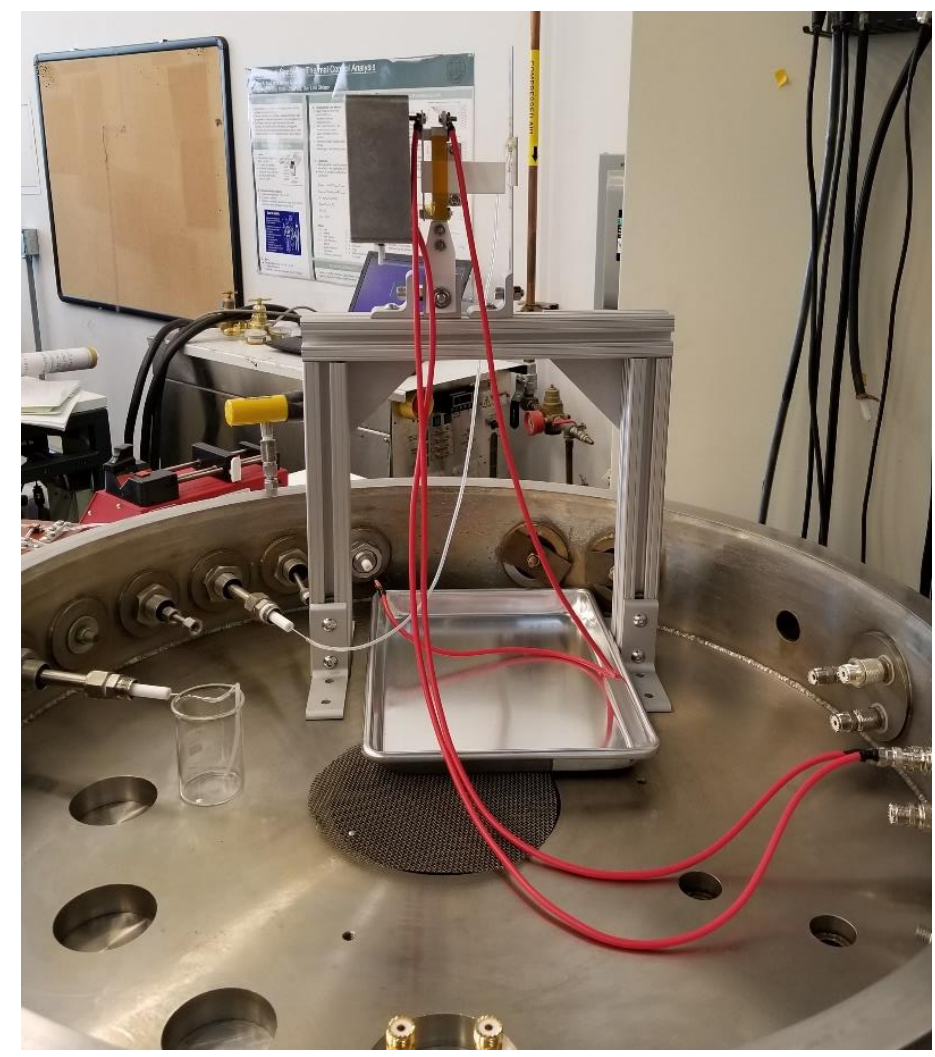

Figure 25: Total assembly of thruster testing setup inside of the vacuum chamber.

\section{Vacuum Chamber Connections and Feedthroughs}

The feedthroughs required for testing of the Research thruster are defined here:

1. The HV power feedthrough used is shown in figure 26. This is a custom-made system, and so is not rated to any particular value, but it has been testing to up to $4.6 \mathrm{kV}$ DC and should theoretically be capable of insulating much higher than $10 \mathrm{kV}$ DC based on the thickness of PEEK and PTFE insulation, as a conservative value. Connection to this feedthrough is made through alligator clips both on the outside and inside. The HV wire that connects the three-way junction from the power supply is 30 " long with a ring terminal on one end and a copper alligator clip on the other. On the connection inside the vacuum chamber, the alligator clip can be secured to the copper rod using selfadhesive silicone tape to remove the possibility of accidental disengagement.

2. The connections carrying the currents to the pico-ammeter are passed through two standard N-type feedthroughs with BNC female adaptors on both the inside and outside of the chamber, as shown in figure 27.

3. Liquid propellant is passed into the chamber using the custom insulated tubing fashioned from Swagelok $1 / 2$ " tubing caps, shown in figure 28 . The stainless steel tubing is $1 / 16$ " in outer diameter $(0.022$ " ID) and seals well against the $1 \mathrm{~mm}$ ID silicone tubing. The tubing is insulated from the chamber and outer $1 / 2$ " tubing using PEEK and PTFE. 


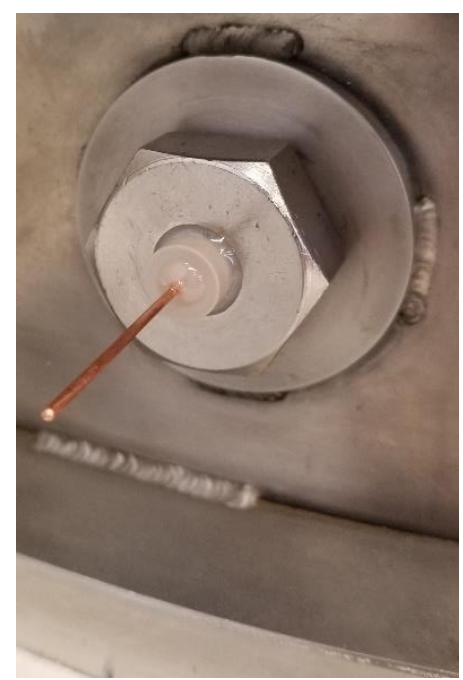

Figure 26: HV feedthrough featuring an electrically insulated 1/16" copper rod.

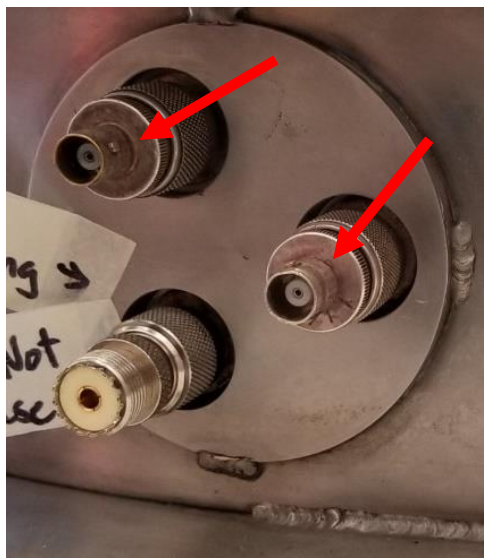

Figure 27: N-type feedthroughs with female BNC adaptors attached.

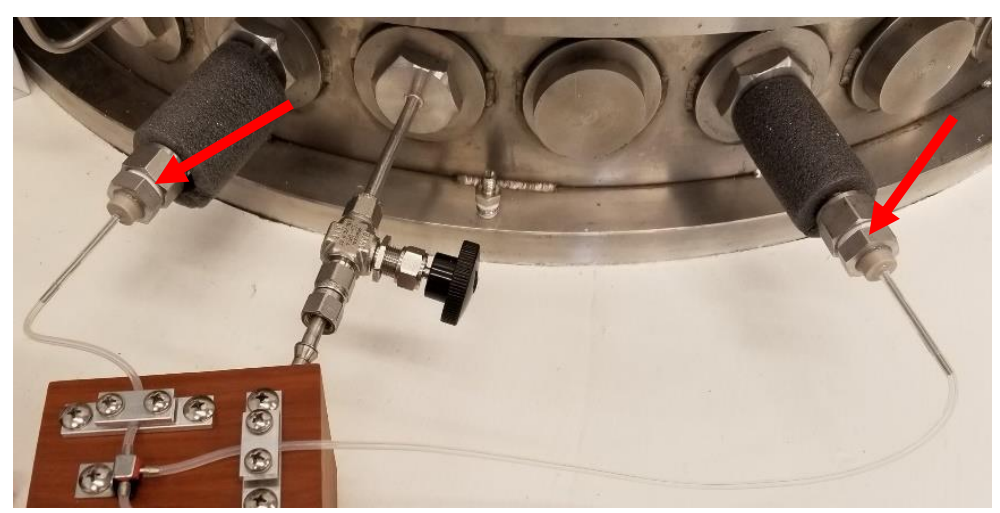

Figure 28: Fluid feedthroughs adapted using existing 1/2" tubing feedthroughs. 


\section{Diagnostic Equipment Connections}

Attachments to the diagnostics equipment are as follows. A simplified electrical diagram is given in figure 29.

1. High voltage is delivered to the thruster using a series of cables and wires, starting an RG-8U cable with a custom input on one end and a male PL-2590 connection on the other. A picture of the custom end is given in figure 9(a). This connects to a male to male PL-259 adaptor and then a 24" RG-8U cable with male PL-259 connections on both ends, where one end of this cable is soldered to a 22 AWG HV wire that leads to a ring terminal. This special cable is shown in figure 9(b). This ring terminal is connected to the wire leading to the main thruster structure using a $1 / 2$ " stainless screw and nut.

2. Also from the high voltage connection is a lead to the HV probe for the oscilloscope. This lead is a $22 \mathrm{AWG} \mathrm{HV}$ wire with ring terminals on both ends. One terminal is connected to the same junction as in the previous step and the other in on the probe. This three-way junction is shown in figure 10. The probe has a BNC cable that connects to the oscilloscope and a ground wire that is connected to ground with an alligator clip.

3. Connections for the pico-ammeter were made using BNC cables connected to the male BNC connectors on the wires connected to the thruster components. The BNC cables used also have male connectors on both sides, so male-to-male BNC adaptors were used. To facilitate easier switching between measuring currents on the extractor and collector surfaces, a shorter BNC cable was connected to the pico-ammeter and was connected to either of the two BNC cables leading to the thruster components. This removes the need to switch out the cables at the back of the equipment rack.

4. Ground connections for the power supply, pico-ammeter, HV probe, and BNC cables are all consolidated to a single mounting point of the equipment rack to avoid ground loop interferences, as seen in figure 11 .

5. Data connections to a computer from the pico-ammeter and oscilloscope are necessary for data collection. The pico-ammeter communicates through RS-232 DB 9 output, so an adaptor going from DB 9 to USB A 2.0 was used to connect with the computer. The oscilloscope communicates through USB B 2.0, so a USB B 2.0 to USB A 2.0 cable was used to connect with the computer. 


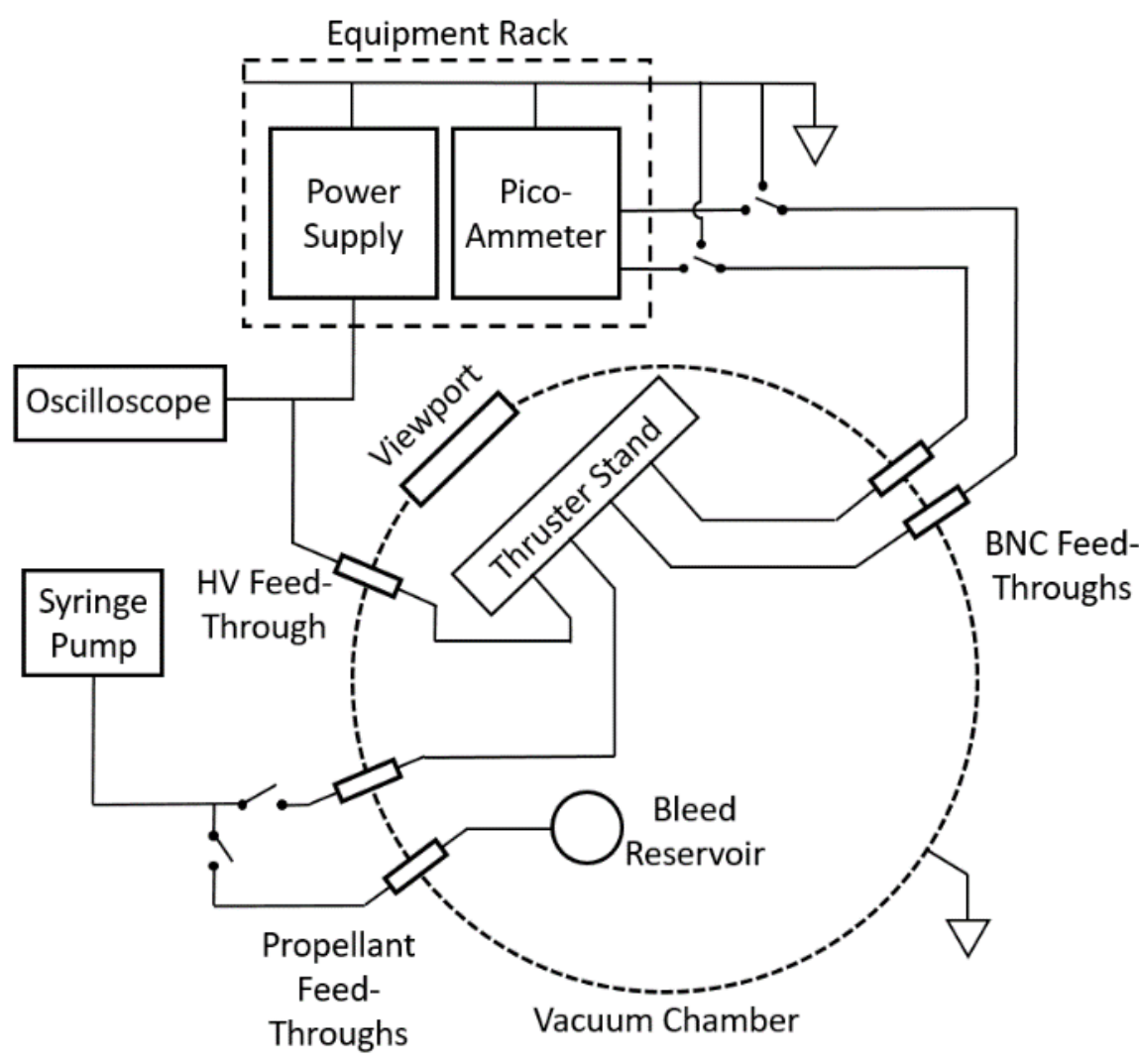

Figure 29: Block diagram of electrical and propellant paths and equipment used for the Research thruster testing.

\section{Diagnostics Equipment Settings and LabView VI}

\section{Power Supply}

The Glassman EL series EL10P04.0 power supply has no additional settings because it is analog only. Note that the voltage output by this power supply does not align with the value displayed on the analog dial, and does not scale linearly.

\section{Pico-Ammeter}

For the data collected in this thesis, the settings used for the Keithley 6487 Pico-ammeter are as follows. Refer to the equipment manual for information about how to operate the pico-ammeter and the effects of these settings. These settings are implemented by the LabView VI.

- RS-232 communication

○ Baud Rate: 9600

- Flow Control: None

- Parity: None

- Data Bits: 8

- Stop Bits: 1.0

- NPLC: 1

- Averaging: Off

- AutoZero: On 
- Damping: On

- AutoRange: Off

- Current Range: 2e-6

\section{Oscilloscope}

For the data collected in this thesis, the settings used for the KeySight Infiniivision DSOX2004A Oscilloscope are as follows. Refer to the equipment manual for information about how to operate the picoammeter.

- Vertical grid step size: $2 \mathrm{kV}$

- Horizontal grid step size: $100 \mu \mathrm{s}$

- Sample Rate: $50 \mathrm{MSa} / \mathrm{s}$

- Acquire Mode: Normal

- Channel Input: DC 1000:1

\section{LabView VI}

A virtual interface was created to control and log data from the pico-ammeter and oscilloscope using LabView. The front panel of this virtual interface is shown in figure 30. The tabs in top left corner are for configuring the equipment through the serial ports, as well as the file save settings. To the right of the configuration tabs are the instantaneous readouts for all values relevant to the data collection and file saving. Three real time plots are generated while the data is being collected so that any irregularities and abnormal system behavior, such as arcing, can be acted upon to prevent damage to the thrusters or the electrical equipment. Data is periodically saved to a Micosoft Excel file after an amount of seconds that can be set in the configuration tabs.

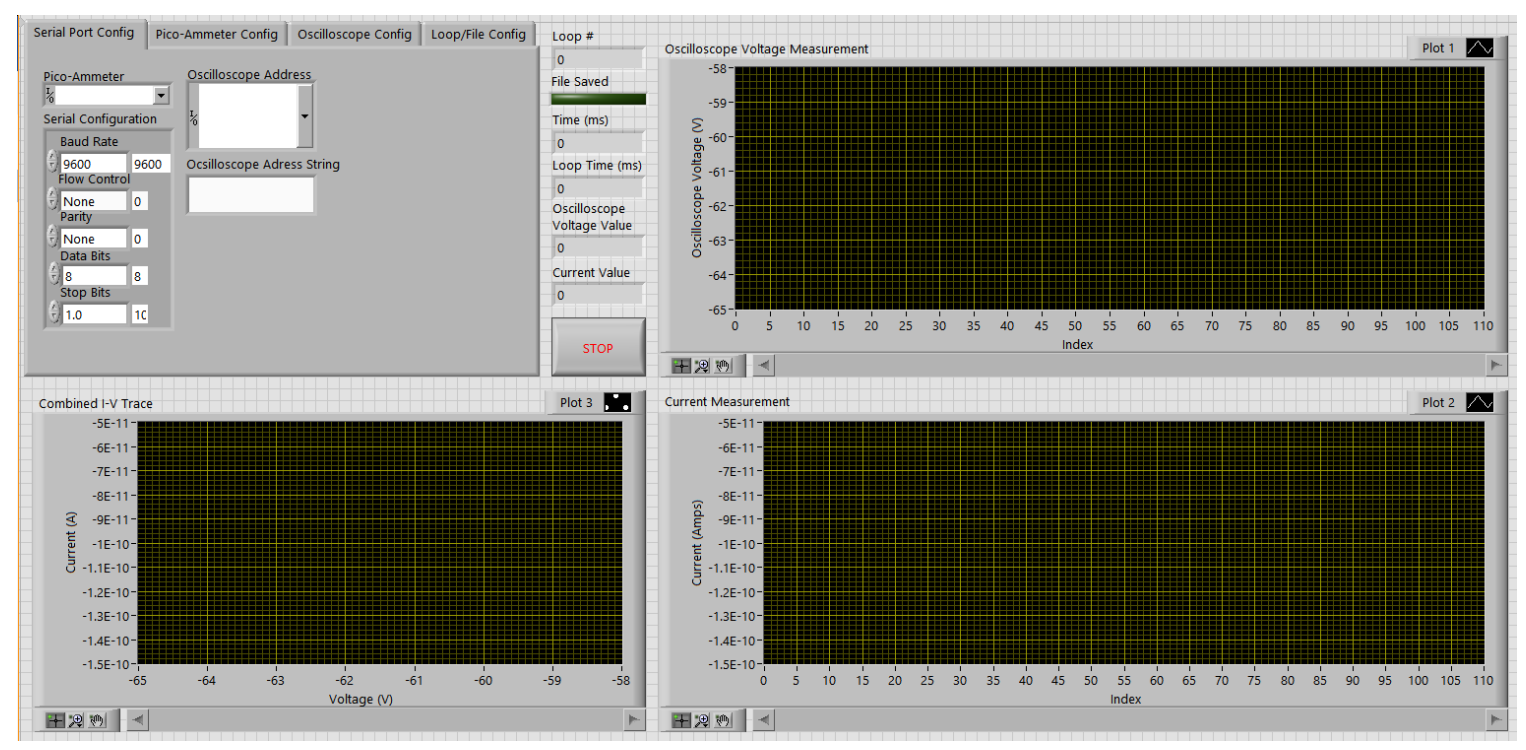

Figure 30: LabView virtual interface front panel used for data collection. 


\section{Standard Operating Procedure for Laboratory Processes}

\section{Process Name:}

Micropropulsion electrospray thruster evaluation using Sodium lodide doped glycerol as propellant.

Purpose: This chemical is to be used as a conductive liquid propellant in an electric micropropulsion thesis project. The technology is known as an electrospray thruster.

Potential Hazards/Toxicity: Sodium lodide, Warning: Causes skin irritation. Causes serious eye irritation. Acute aquatic toxicity. Very toxic to aquatic life with long lasting effects.

Ethanol, Danger: Highly flammable liquid and vapor. Causes serious eye irritation. Causes damage to organs. Causes damage to organs through prolonged or repeated exposure.

\section{Engineering Controls:}

Open area with good ventilation.

\section{Personal Protective Equipment (PPE)-}

\section{Hand Protection:}

Standard nitrile gloves will be worn during procedure.

\section{Eye Protection:}

Safety glasses will be worn at all times during procedure.

\section{Skin and Body Protection:}

Lab personnel working with the chemicals will wear full-length pants or its equivalent, closed-toe footwear with no skin being exposed, and a lab coat.

Hygiene Measures:

Wash hands after working with the hazardous substances and when leaving the lab/shop. 
Respirators may be required under any of the following circumstances:

- As a last line of defense (i.e., after engineering and administrative controls have been exhausted).

- When Permissible Exposure Limit (PEL) will or may be exceeded ${ }_{2}$ or the airborne concentration is unknown.

- Regulations require the use of a respirator.

- There is potential for harmful exposure due to an atmospheric contaminant (in the absence of PEL)

- As PPE in the event of a chemical spill clean-up process

Prior to obtaining a respirator, an exposure assessment of the process or procedure must be conducted. If respiratory protection is required, then lab personnel must obtain respiratory protection training, a medical evaluation, and a respirator fit test through EH\&S. This is a regulatory requirement.

\section{First Aid Procedures for Chemical Exposures}

If inhaled:

Evacuate the victim to a safe area as soon as possible. Loosen tight clothing such as a collar, tie, belt or waistband. If breathing is difficult, seek medical attention. If the victim is not breathing, perform mouth-to-mouth resuscitation. WARNING: It may be hazardous to the person providing aid to give mouth-to-mouth resuscitation when the inhaled material is toxic, infectious or corrosive. Seek immediate medical attention.

In case of skin contact:

In case of contact, immediately flush skin with plenty of water for at least 15 minutes while removing contaminated clothing and shoes. Cold water may be used. Wash clothing before reuse. Thoroughly clean shoes before reuse. Get medical attention, as necessary.

In case of eye contact:

Immediately flush eyes with plenty of water for at least 15 minutes. Check for and remove any contact lenses. Get medical attention.

\section{If swallowed:}

No information.

\section{Special Handling and Storage Requirements}

Avoid contact with skin and eyes. Avoid formation of dust and aerosols. Provide appropriate exhaust ventilation at places where dust is formed. Keep container tightly closed in a dry and well ventilated place. Air, light, and moisture sensitive. Storage class (TGRS 510): 13: Non Combustible Solids.

\section{Spill and Accident Procedure}

\section{Chemical Spill Dial 911 and 756-6661}

Spill - Assess the extent of danger. Help contaminated or injured persons. Evacuate the spill area. Avoid breathing vapors. If safe, confine the spill to a small area using a spill kit or absorbent material. Keep others from entering contaminated area (e.g., use caution tape, barriers, etc.). 
Small $(<\mathbf{L}$ ) - If you have training, you may assist in the clean-up effort. Use appropriate personal protective equipment and clean-up material. Double bag spill waste in plastic bags, label and arrange hazardous waste pick-up.

Large (>1 L) - Evacuate spill area. Dial 911 and EH\&S at 756-6661 for assistance. Remain available in a safe, nearby location for emergency personnel.

Chemical Spill on Body or Clothes - Remove clothing and rinse body thoroughly in emergency shower for at least 15 minutes. Seek medical attention. Notify supervisor, advisor or P.I. immediately.

Chemical Splash Into Eyes - Immediately rinse eyeball and inner surface of eyelid with water from the emergency eyewash station for_a minimum of 15 minutes by forcibly holding the eye open. Seek medical attention. Notify supervisor, advisor or P.I. immediately.

\section{Medical Emergency Dial 911 or 756-6661}

Life Threatening Emergency, After Hours, Weekends And Holidays - Dial 911

Note: All serious injuries must be reported to Supervisor/PI within 8 hours. Note: Any and all loss of consciousness requires a 911 call

\section{Non-Life Threatening Emergency -}

- Students: Seek medical attention at the campus Health Center M, T, Thu, Fr 8:00 am 4:30 pm and $W$ 9:00 am - 4:30 pm

- Emergency Medical services in the community are available at any time at hospital emergency rooms and some emergency care facilities.

\section{All injuries must be reported to PI/Supervisor immediately and follow campus injury} reporting. Follow procedures for reporting of student, visitor injury on the EH\&S website at: http://afd.calpoly.edu/riskmgmt/incidentreporting.asp

- Paid staff, students, faculty: seek initial medical attention for all non-life threatening injuries at:

> MED STOP, 283 Madonna Road, Suite B (next to See's Candy in Madonna Plaza)

(805) 549-8880 Hours: M-F 8a - 8p; Sat/Sun 8a - 4p

$>$ After MED Stop Hours: Sierra Vista Hospital Emergency Room

1010 Murray Avenue (805) 546-7651, Open 24 hours

\section{All injuries must be reported to PI/Supervisor immediately and follow campus injury reporting for employee injuries (Workmen's Comp.). Follow procedures on the EH\&S website at: http://afd.calpoly.edu/riskmgmt/incidentreporting.asp}

Needle stick/puncture exposure (as applicable to chemical handling procedure) - Wash the affected area with antiseptic soap and warm water for 15 minutes. For mucous membrane exposure, flush the affected area for 15 minutes using an eyewash station. Seek medical attention. Note: All needle stick/puncture exposures must be reported to supervisor, advisor or P.I. and EH\&S office immediately. 


\section{Decontamination/Waste Disposal Procedure}

\section{General hazardous waste disposal guidelines:}

\section{Label Waste}

- Affix a hazardous waste tag on all waste containers as soon as the first drop of waste is added to the container. Generic waste labels can be found here: http://afd.calpoly.edu/ehs/docs/hazwaste label template.pdf

\section{Store Waste}

- Store hazardous waste in closed containers, in secondary containment and in a designated location

- Double-bag dry waste

- Waste must be under the control of the person generating \& disposing of it

\section{Dispose of Waste}

- Dispose of regularly generated chemical waste as per guidelines on EH\&S website at: http://afd.calpoly.edu/ehs/docs/csb no6.pdf

- Prepare for transport for pick-up. Use secondary containment.

Call EH\&S at 756-6661 for questions.

\section{Empty Containers-}

- Dispose as hazardous waste if container once held extremely hazardous waste (irrespective of the container size) A list can be found at: http://afd.calpoly.edu/ehs/docs/extremely hazardous wastes.pdf

- All other containers are legally empty once a concerted effort is made to remove, pour out, scrape out, or otherwise completely empty the vessel. These may be disposed of as recycling or common trash as appropriate.

\section{Safety Data Sheet (SDS) Location}

Online SDS can be accessed at: https://www.sigmaaldrich.com/

Physical copies can be found in the Safety binder inside the Building 41B Room 137, and attached to this procedure.

\section{Protocol/Procedure}

The specific procedure for loading, testing with, and cleaning the propellant is as follows:

Note: Cleaning as called for and referenced in this procedure may include the use of denatured alcohol as a solvent. Alcohol is highly flammable and vaporizes quickly. Avoid open flames, hot surfaces, and surfaces with the potential of electrical discharge. 
1. Set up and prepare testing apparatus inside vacuum chamber, as detailed in the project thesis. Ensure all fluid feedthroughs and tubes are properly connected. Close chamber.

2. Confirm the PPE outlined in this SOP is being worn correctly.

3. Remove the container of the chemical (Sodium lodide) from its storage (flammables cabinet in Building 41C Room 144) and move to the laboratory area where the preparation and testing is conducted (Building 41B Room 137).

4. Secure a safe area in the vicinity to conduct this procedure. If others are present in the area, warn them to keep a safe distance, defined as beyond an arms-length away to prevent accidental contact. All others in the room will wear safety glasses at all times. Ideally, conduct this procedure when others are not present in the laboratory.

5. Ensure the area is adequately vented by opening both the East and West doors of the room. Put signage to inform others to keep the doors open at all times during this procedure.

6. Have adequate materials on hand to contain the chemical as this procedure is being conducted, including paper towels and Kim-wipes. Ensure the provided proper waste disposal container is on-hand or nearby to minimize the time and distance any potential waste is outside the container.

7. Weigh an empty mixing container on scale to obtain the dry weight.

8. Fill the mixing container with approximately $8 \mathrm{~mL}$ of glycerol. Weigh to obtain precise weight of glycerol.

9. Carefully open the Sodium lodide container.

10. Measure out the appropriate weight of the chemical to add to the glycerol ( $20 \%$ by weight).

11. Add the Sodium lodide to the glycerol in the mixing container and begin mixing.

12. Once thoroughly mixed, insert the syringe needle and begin loading the chemical into the barrel of the syringe. Approximately 5 to $7 \mathrm{~mL}$ should be loaded into the gas-tight $10 \mathrm{~mL}$ glass syringe. During loading it may be necessary to depress the syringe plunger to attempt to remove air bubbles present in the barrel. When doing this, expel the air and chemical into an absorbent material and properly dispose of it in hazardous waste.

13. When done loading, carefully remove the syringe needle from the container and wipe away the excess liquid on the syringe needle and dispose of the waste properly.

14. Return the chemical to its proper storage area.

15. When ready to begin testing, secure the syringe inside of the syringe pump, outside of the vacuum chamber. Remove the cap on the needle and connect the syringe to the silicone tubing leading to the vacuum chamber feedthroughs.

16. Replace gloves as a precaution to prevent contamination of testing equipment while operating. Do not contact the syringe or fluid feedthroughs unless necessary to prevent contamination.

17. Begin chamber operation procedure for testing. Chamber operation is detailed in the documents located near the chamber and in a repository inside the laboratory. During the testing process, the chemical is contained inside the chamber.

18. After testing is concluded, ensure the required PPE is again worn.

19. Vent the vacuum chamber to atmosphere. Open the chamber and begin cleaning any spillage inside if necessary.

20. Carefully remove the testing apparatus from the chamber and begin disassembly and cleaning the individual components. Use dry wipes to collect accumulated amounts of the chemical, then use denatured alcohol soaked wipes as a final clean. Repeat cleaning until all surfaces are free of residue and have been wiped down with alcohol a final time. Dispose of the waste into the proper hazardous waste container as necessary. 


\section{NOTE:}

Any deviation from this SOP requires approval from $\mathrm{PI}$.

Date: 5/7/2019 P.I. or Supervisor: Dr. Amelia Greig 
Documentation of Training (signature of all users is required)

- The Principal Investigator must ensure that his/her laboratory personnel have attended appropriate laboratory safety training or refresher training within the last one year.

- $\quad$ Training must be administered by PI or Lab Manager to all personnel in lab prior to start of work with particularly hazardous substance or newly synthetic chemical listed in the SOP.

- Refresher training will need to be provided when there is a change to the work procedure, an accident occurs, or repeat non-compliance.

I have read and understand the content, requirements, and responsibilities of this SOP:

\begin{tabular}{|l|l|l|}
\hline Name & Signature & Date \\
\hline & & \\
\hline & & \\
\hline & & \\
\hline & & \\
\hline & & \\
\hline & & \\
\hline & & \\
\hline & & \\
\hline & & \\
\hline
\end{tabular}

